\title{
Application of Artificial Intelligence in Three Phase Unbalanced Smart Power Distribution Grid
}

\author{
Deepak Tiwari \\ WVU, detiwari@mix.wvu.edu
}

Follow this and additional works at: https://researchrepository.wvu.edu/etd

Part of the Power and Energy Commons

\section{Recommended Citation}

Tiwari, Deepak, "Application of Artificial Intelligence in Three Phase Unbalanced Smart Power Distribution Grid" (2021). Graduate Theses, Dissertations, and Problem Reports. 8005.

https://researchrepository.wvu.edu/etd/8005

This Dissertation is protected by copyright and/or related rights. It has been brought to you by the The Research Repository @ WVU with permission from the rights-holder(s). You are free to use this Dissertation in any way that is permitted by the copyright and related rights legislation that applies to your use. For other uses you must obtain permission from the rights-holder(s) directly, unless additional rights are indicated by a Creative Commons license in the record and/ or on the work itself. This Dissertation has been accepted for inclusion in WVU Graduate Theses, Dissertations, and Problem Reports collection by an authorized administrator of The Research Repository @ WVU.

For more information, please contact researchrepository@mail.wvu.edu. 


\title{
Application of Artificial Intelligence in Three Phase Unbalanced Smart Power Distribution Grid
}

\author{
Deepak Tiwari
}

\author{
Dissertation submitted \\ to the Benjamin M. Statler College of Engineering and Mineral Resources \\ at West Virginia University \\ in partial fulfillment of the requirements for the degree of \\ Doctor of Philosophy \\ in \\ Electrical Engineering \\ Jignesh Solanki, Ph.D., Chair \\ Sarika Khushalani Solanki, Ph.D. \\ Muhammad A. Choudhry, Ph.D. \\ Matthew Valenti, Ph.D. \\ Hong-Jian Lai, Ph.D. \\ Lane Department of Computer Science and Electrical Engineering \\ Morgantown, West Virginia \\ 2021
}

Keywords: Electric Vehicles, Artificial Intelligence Techniques, Convolutionary Neural Network, Distributed Resource Allocation, Distribution System

Copyright 2021 Deepak Tiwari 


\section{ABSTRACT \\ Application of Artificial Intelligence in Three Phase Unbalanced Smart Power Distribution Grid \\ Deepak Tiwari}

Electrification of the transportation sector can play an essential role in curbing fossil fuel scarcity and oil shortages in the world. Electric vehicles (EVs) can significantly reduce CO2 emissions, improve urban pollution. Apart from these advantages of EVs, they may also pose challenges to the distribution grid. Increasing penetration of EVs puts an extra burden and leads to affect the grid severely. Load congestions, voltage drops/regulation, overloading are some of the issues that might incur in the grid because of improper charging of EVs. The uncertain nature of EV owners makes it very difficult to predict the charging pattern. So the grid operator may face a daunting task to avoid overloading if huge EVs are charged without following any smart charging management strategies. We propose a framework that allows the EV users to follow a well-coordinated charging schedule and fulfill various objectives like load variance minimization and social welfare maximization for both electric utility and EV owners. A proof of concept of distributed resource allocation for EV charging is implemented using output consensus approach. Microgrids are components of a smart power distribution grid that can operate as a decentralized/localized grid when disconnected from the main grid. A microgrid can enhance the grid resiliency and provide adequate power to a community or a region in case of grid failure caused by natural disasters or any other disruptions. We also propose the optimal scheduling model for Distributed Energy Resources (DERs) including an EV charging station and battery energy storage system (BESS) in a community microgrid. This model also minimizes the cost incurred in operating the DERs, charging station, BESS, and the grid load. Due to the two-way power flow in the smart grid because of the EVs and distributed energy resources (DERs) involvement, the distribution system operator (DSO) must provide control protection to equipment and manage the reverse power flow without violating system constraints. Artificial Intelligence (AI) leads the path for a promising smart grid future and helps the DSO tackle such challenges. Given the large size of data flow in the smart grid due to phasor measurement units (PMUs) and smart sensors, AI-based techniques help the grid operator to manage and analyze such data effectively and improve grid resiliency. Machine 
learning can help the grid operator forecast the uncertainty in supply-demand because of the integration of renewable energy sources. For system planning and operation, the utility needs to perform the load flow analysis in regular intervals to check the different network quantities like bus voltages, line currents, real and reactive powers to plan for the future in case of hypothetical critical conditions like system failure or fault analysis. Such load flow analysis can be performed using different methods, including iterative power flow analysis like the backward forward sweep method. This dissertation develops an AI-based model for a three-phase unbalanced smart grid with a range of EV penetration, renewable energy sources like photovoltaic systems (PVs), and wind energy. We present a trained deep neural network that predicts the branch currents, node voltages, angles, power losses with very high accuracy. Our trained deep neural networks will replace the need for an iterative-based power flow. The proposed models are validated on American Electric Power utility feeder models and various IEEE benchmark distribution test feeders. 


\section{ACKNOWLEDGEMENT}

There are numerous people to whom I would like to thank for the unconditional support throughout my Ph.D. journey. First and foremost, I would like to begin with my advisor Dr. Jignesh Solanki for accepting me as his Ph.D. student. Without his zeal to always help and guide me, this research would not have completed. I can not express enough my gratitude to him for always pushing me to aim higher and learn well in this journey. I feel grateful for the freedom he gave for me to work on the ideas and his readiness to always listen to my views. His passion for taking up new challenges and a positive intent in his actions is something motivated me in this journey.

I also would like to thank my co-advisor Dr. Sarika Solanki for her very valuable feedback throughout my research. Her expertise in Distribution system helped me a lot to finish this research. I would like to express my gratitude to Dr. Muhammad A. Choudhry, Dr. Matthew Valenti, and Dr. Hong-Jian Lai for accepting to be in my committee and providing the valuable comments and suggestions which helped me improve my research. I also want to thank the Lane department for supporting me as a graduate student.

I would like to sincerely thank Dr. Sushama Wagh and Dr. Navdeep Singh who have always been my source of motivation and one of the reasons why I could come to West Virginia University.

No journey is complete without the friends. I wish to specially thank all my friends Venkatesh Chinde, Veeru Talreja, Vishal Verma, Mohammad Reza Khalghani, Hasan Ul Banna, Anusha Kan-

dula for making my journey at WVU a smooth and memorable one. I learned a lot from the very productive discussions we had. Thank you for the help and support.

I would like to thank my entire family: parents for believing in me, my sisters, brother in-laws for your unconditional love and support throughout my journey. I am sorry to have missed good and bad times with you all.

At the end, I would like to wholeheartedly thank my lovely wife Shruti, without her encouragement, love, and support, this journey would not be possible. 


\section{TABLE OF CONTENTS}

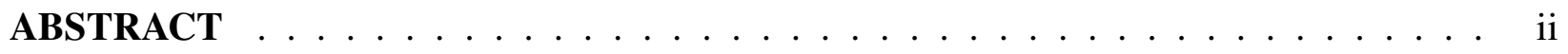

ACKNOWLEDGEMENT $\ldots \ldots \ldots \ldots \ldots \ldots \ldots \ldots$ iv

LIST OF FIGURES $\ldots \ldots \ldots \ldots \ldots \ldots \ldots \ldots$ vii

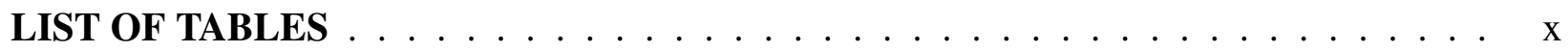

1 Introduction 1

2 Optimal Scheduling of Electric Vehicles and Load Flow Analysis 5

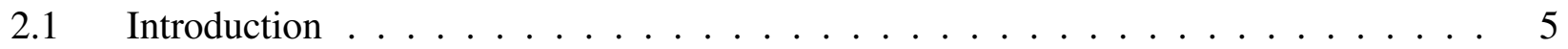

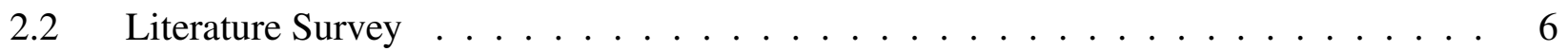

2.3 Charging Coordination of Aggregated Electrical Vehicles for Load Variance Minimization in Distribution System . . . . . . . . . . . . . . . . . . 8

2.3 .1 Problem Formulation $\ldots \ldots \ldots \ldots$

2.3.2 Load Flow Analysis . . . . . . . . . . . . . . . . . . . . 9

2.4 Optimal Scheduling of EV Charging Station and DERs including BESS in the Community Microgrid . . . . . . . . . . . . . . . . . . . . . 10

2.4 .1 System Model . . . . . . . . . . . . . . . . . . . . . . . . . . 10

2.4 .2 Optimization problem . . . . . . . . . . . . . . . . . . . 13

2.5 Social Welfare problem for Electric Vehicle Charging . . . . . . . . . . . . . 13

2.6 Results . . . . . . . . . . . . . . . . . . . . . . . . . . . 17

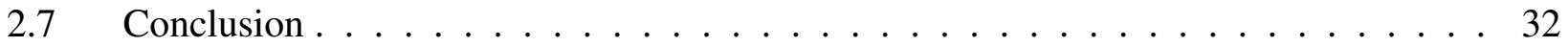

3 Vehicle-to-Grid Integration for enhancement of Grid: A Distributed Resource Allocation Approach $\quad 34$

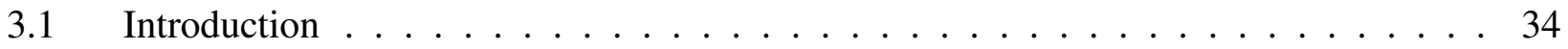


3.2 Literature Survey . . . . . . . . . . . . . . . . . . . . . . . . 34

3.3 Problem Statement and Analogies ． . . . . . . . . . . . . . . . . 37

3.4 Preliminaries for DRA approach and barrier function formulation . . . . . . . . . 38

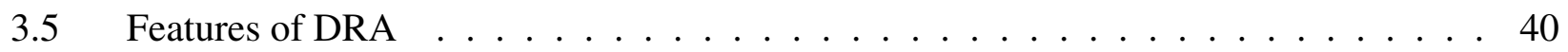

3.6 Application of DRA for load management of PEV . . . . . . . . . . . . . 43

3.7 Representative Case study and Results for grid enhancement using PEVs . . . . . . . 47

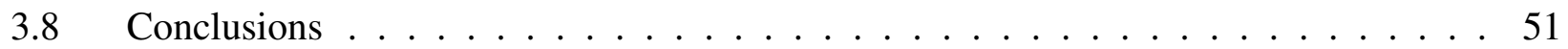

$4 \quad$ Power Flow Analysis using Deep Learning Techniques in a Three Phase Unbalanced Distribution Network

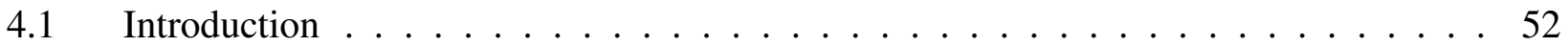

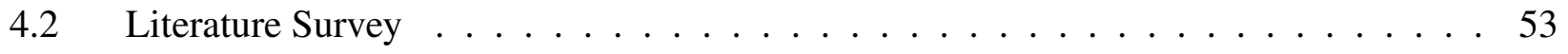

4.3 Distribution Power Flow $\ldots \ldots \ldots \ldots$

4.4 Data Analysis . . . . . . . . . . . . . . . . . . . . 56

4.5 Proposed Methodology $\ldots \ldots \ldots \ldots$

4.6 Neural Network Model . . . . . . . . . . . . . . . . . . . . . . . . . . . . . . . . 61

4.7 Simulation Results . . . . . . . . . . . . . . . . . . . . . . . . . . 69

4.8 Case Studies . . . . . . . . . . . . . . . . . . . . . . . . . . . . . . . 79

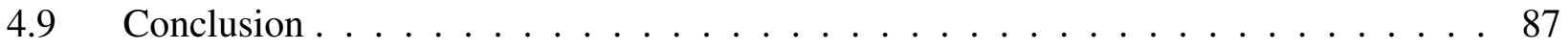

$5 \quad$ Conclusion and Proposed Future Work $\quad 88$

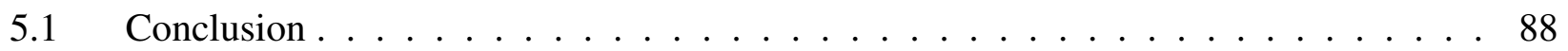

5.2 Proposed Future Work . . . . . . . . . . . . . . . . . . . . . . . . . 89

LIST OF PUBLICATIONS $\ldots \ldots \ldots \ldots$

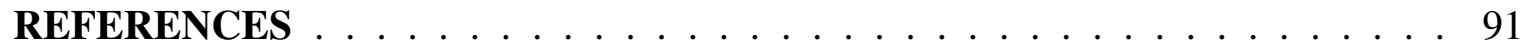

\section{Appendices}

Appendix A Abbreviation

103 


\section{LIST OF FIGURES}

1.1 Smart Grid and Components . . . . . . . . . . . . . . . . 3

2.1 Community Microgrid . . . . . . . . . . . . . . . . 11

2.2 IEEE 13 Node Test System . . . . . . . . . . . . . . . . . . 17

2.3 Load Profile . . . . . . . . . . . . . . . . . . . . . . . . 19

2.4 Charging Profile in Vehicle to Grid Mode . . . . . . . . . . . . . . . . . 19

2.5 SOC in Vehicle to Grid Mode . . . . . . . . . . . . . . . . . . . 20

2.6 Charging Profile in Grid to Vehicle Mode . . . . . . . . . . . . . . 21

2.7 SOC in Grid to Vehicle Mode . . . . . . . . . . . . . . . . . . 21

2.8 Load Variance Minimization in Vehicle to Grid Mode at (a) Node 646 (b) Node 611 (c) Node 634 (d) Node $675 \ldots$. . . . . . . . . . . . . . . . . . . 22

2.9 Load Variance Minimization in Grid to Vehicle Mode at (a) Node 646 (b) Node 611 (c) Node 634 (d) Node $675 \ldots$. . . . . . . . . . . . . . . . . 23

2.10 Voltage during Vehicle to Grid Mode at (a) Node 646 - Node 611 (b) Node 634 Node $675 \ldots \ldots \ldots \ldots \ldots \ldots$. . . . . . . . . . . . . . . . . . . . . . . . . .

2.1124 -hour Feeder Load Profile . . . . . . . . . . . . . . . . . 25

2.12 Market Forecast for 24 hours . . . . . . . . . . . . . . . . 25

2.13 Optimal DER Dispatch Schedule . . . . . . . . . . . . . . 27

2.14 Optimum BESS Charge/Discharge Schedule . . . . . . . . . . . . . 28

2.15 Aggregated Optimal Charge/Discharge Schedule for EV Charging Station . . . . . 28

2.16 Optimum Grid Dispatch Schedule . . . . . . . . . . . . . . . . . . 29

2.17 Control Trajectories for $10 \mathrm{EVs}$. . . . . . . . . . . . . . 30

2.18 Control Trajectories for $100 \mathrm{EVs} \ldots \ldots$. . . . . . . . . . . 30

2.19 Comparison of Charging Demand with and without Energy Management . . . . . 31

2.20 IEEE 34 Node Test Case $[53] \ldots \ldots \ldots \ldots$. . . . . . . . . . . . . 31 
2.21 Voltage Profile at the Node 860,844 , and $890 \ldots \ldots 33$

3.1 The connection of Power Grid with the transient and fixed population. . . . . . . 38

3.2 Representation of (3.6) and (3.7) via feedback interconnection outlook. . . . . . . 42

3.3 Partial information connectivity diagram for a system of three PEVs connected to the grid with grid providing payoff functions for four time slots. . . . . . . . . . 45

3.4 Flowchart for implementation of DRA approach in grid profile enhancement using PEVs . . . . . . . . . . . . . . . . . . . . . . . . . 47

3.5 Cumulative load profiles $\ldots \ldots \ldots \ldots$

3.6 PEV charging and discharging profiles $\ldots \ldots \ldots \ldots \ldots$

3.7 Charging strategies for different PEVs . . . . . . . . . . . . . . . . 49

3.8 Variation in design parameters $\ldots \ldots \ldots \ldots \ldots$

3.9 Effect of number of PEVs . . . . . . . . . . . . . . . . . . 50

4.1 Relation Between Machine Learning and Data Science $\ldots \ldots \ldots 3$

4.2 Big Data in Power System . . . . . . . . . . . . . . . 58

4.3 Outline of Proposed Methodology $\ldots \ldots \ldots$. . . . . . . . . . 60

4.4 Problem Architecture . . . . . . . . . . . . . . . . . . . . . . 61

4.5 Multi Layer Perceptron . . . . . . . . . . . . . . . . . . . 62

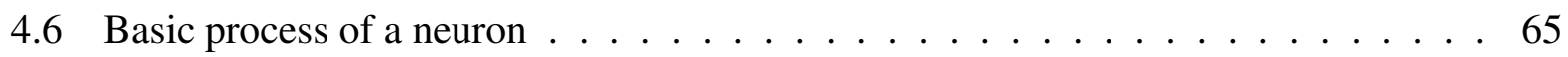

4.7 Mathematical model of a biological neuron $\ldots \ldots \ldots \ldots$

4.8 Radial Basis Function Network . . . . . . . . . . . . . . . . . . . 68

4.9 IEEE 4 Node Test Model $\ldots \ldots \ldots$

4.10 Loadshape . . . . . . . . . . . . . . . . . . . . . . 70

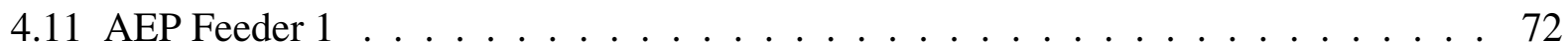

4.12 CNN sequence for AEP feeder $\ldots \ldots \ldots 72 \ldots \ldots \ldots \ldots$

4.13 Normalized Output Voltage . . . . . . . . . . . . . . . . . . . 73

4.14 Normalized Output Current . . . . . . . . . . . . . . . . . . . . . . 73

4.15 Normalized Output Voltage Angle . . . . . . . . . . . . . . . . . 74

4.16 Normalized Output Current Angle . . . . . . . . . . . . . . . . . . 74

4.17 Performance Evaluation- MAE . . . . . . . . . . . . . . . . . . 75

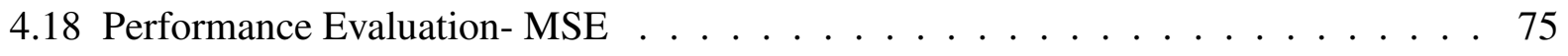


4.19 IEEE 123 Bus Test Case Feeder $\ldots \ldots \ldots$. . . . . . . . . . . 76

4.20 Performance Evaluation- MAE . . . . . . . . . . . . . . . . . . 77

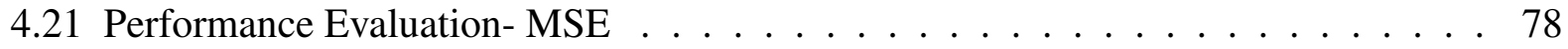

4.22 Circuit plot when topology changes _ . . . . . . . . . . . . . . . 79

4.23 Mixing Training and Testing Data $\ldots \ldots \ldots . \ldots \ldots$

4.24 MAE- Mixing the Training Data $\ldots \ldots \ldots . \ldots \ldots 1$

4.25 MAE- Dropout $\ldots \ldots \ldots \ldots$. . . . . . . . . . . . . 81

4.26 Voltage for OpenDSS(GT) $\ldots \ldots \ldots 2$

4.27 Voltage for OpenDSS $(\mathrm{GT})$ vs AI $\ldots \ldots \ldots \ldots$. . . . . . . . . . 82

4.28 Block Diagram of the PV System Model . . . . . . . . . . . . . . . . . 83

4.29 MAE- PV Integration . . . . . . . . . . . . . . . . . . . . 84

4.30 MSE- PV Integration . . . . . . . . . . . . . . . . . . 84

4.31 OpenDSS(GT) Currents . . . . . . . . . . . . . . . . 85

4.32 Currents for OpenDSS(GT) vs AI . . . . . . . . . . . . . . . . . 85

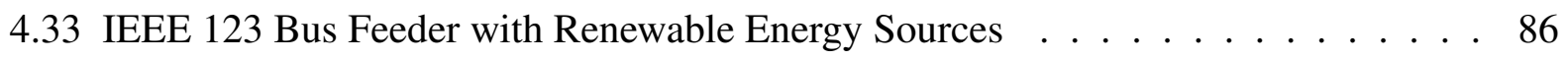




\section{LIST OF TABLES}

2.1 System Configuration . . . . . . . . . . . . . . . . 18

2.2 Load Data . . . . . . . . . . . . . . . . . . . . . 18

2.3 Voltage Unbalance at All Nodes . . . . . . . . . . . . . . . . . 24

2.4 DER Data . . . . . . . . . . . . . . . . . . . . 26

2.5 Load Data . . . . . . . . . . . . . . . . . . . . 32

4.1 FBS summary $[108] \ldots \ldots \ldots \ldots \ldots \ldots$

4.2 Performance Comparison Results . . . . . . . . . . . . . . . . 78

4.3 Prediction Error . . . . . . . . . . . . . . . . . . . . 87 


\section{CHAPTER 1}

\section{Introduction}

The current electric grid worldwide is aging and working at more than its capacity in most of the places. With the increasing participation of distributed energy resources (DERs) like and demand response, the traditional grid is shaping into the smart grid. DERs such as renewable energy sources like photovoltaic cell, wind farms, combined heat and power generation (CHP), battery energy storage system, and electric vehicles, contribute to a more reliable, greener, and a smarter grid. The smart grid is equipped with two way-communication and more reliable than the traditional one. The smart grid is decentralized in a sense that it can distribute power to help in balancing the load, decrease peak loads, and the power outages. The modern power grid needs to be able to fulfill peak load demand for more time slots which increases the cost and global warming due to the dependence on non renewable sources. Smart grid uses the smart sensors like phasor measurement units, smart meters at the consumer end which allows the consumer to interact with the grid and felicitate better exchange of information. Some of the advantages of a smart grid include [1]:

- Self Healing: Smart grid's ability to continuously self assess using the smart sensors can help the grid in self healing and minimize the impacts of blackouts.

- Smart grid allows better integration of small and large scale renewable energy generation.

- The transmission and distribution losses are reduced and thus improving the efficiency of the smart grid.

- Peak load reduction.

- Advanced control and security measures.

Apart from these, smart grid has a lot of other advantages like reduced pricing for customer and a low operation costs for utilities. A microgrid is gaining attention to overcome such problem. A microgrid is a building block of smart grids. The US Department of Energy defined Microgrid as "a group of interconnected loads and distributed energy resources within clearly defined electrical boundaries that acts as a single controllable entity concerning the grid. A microgrid can connect 
and disconnect from the grid to enable it to operate in both grid-connected or island mode" [2]. DERs consist of distributed generation, battery energy storage system, demand response technologies, plays an important role in curtailing peak load, providing ancillary services to the microgrid. The advancement of smart grid, integration of renewable energy sources to the grid, electric vehicles and charging station, all help the grid to perform demand-side management (DSM) which eventually reduce the operating cost, power losses, peak shaving, control the global warming and many more advantages. Renewable energy sources (RES) bring the uncertainty to the microgrid because of intermittent electricity generation.

The threat of global warming forcing the world to adapt green technologies. The power and transportation industries are responsible for more than 60 percent of greenhouse gas emissions [3]. The availability, rising prices of fuel, $\mathrm{Co} 2$ emissions are amongst the various factors that lead to a growing number of electric vehicles (EVs). The electric vehicles can be classified into two main categories: Battery EVs (BEV), Hybrid electric vehicles (HEVs), Plug-in electric vehicles (PEVs). Plug-in hybrid electric vehicles (PHEVs) are a subset of HEVs which has larger battery storage and their batteries can be charged at any charging station through the power grid. PHEVs are the other types of electric vehicles which are catching the attention of researchers. However, it has been shown in [4] that the significant increment of EVs contribute to grid stress and may create several problems like overloading, system losses, congestion, voltage regulation. Properly coordinated charging is necessary to mitigate these issues in the grid [5]. The optimized coordinated charging of PHEVs would enhance the power system efficiency by playing a major role in reducing uncertainty due to the use of renewable energy resources, demand-side management, and spinning reserve services.

Figure 1.1 represents the smart grid with various components. PHEVs can work as a moveable power source. The batteries of PHEVs can be charged from the grid during the off-peak hours and can provide the energy deposited in the batteries to the grid during the peak hours. This function is known as Vehicle to Grid technology (V2G) [6]. In V2G operation, the stored energy is sent back to the grid. EV can be used as either load or storage or both which makes it an important agent in grid operations. Choosing a V2G option can enhance the security, reliability of the grid and can significantly reduce load variance, or maximize load factor which in turn would reduce the overall cost and hence improve efficiency. V2G implementation is possible only if it's operated under some planned strategies and consider the incentives given to the EV owners. The main aim of $\mathrm{V} 2 \mathrm{G}$ operation is to provide optimal charging schedule of EV, also making sure the vehicle has enough energy before it reaches another charging station. In V2G mode, The control center receives the signals from the power grid and gives the optimal charging profile for the vehicle. The charging station also sends the vehicle information like battery type, vehicle model, State of charge information of the PHEVs, back to the control center. Also, control center must know some key 


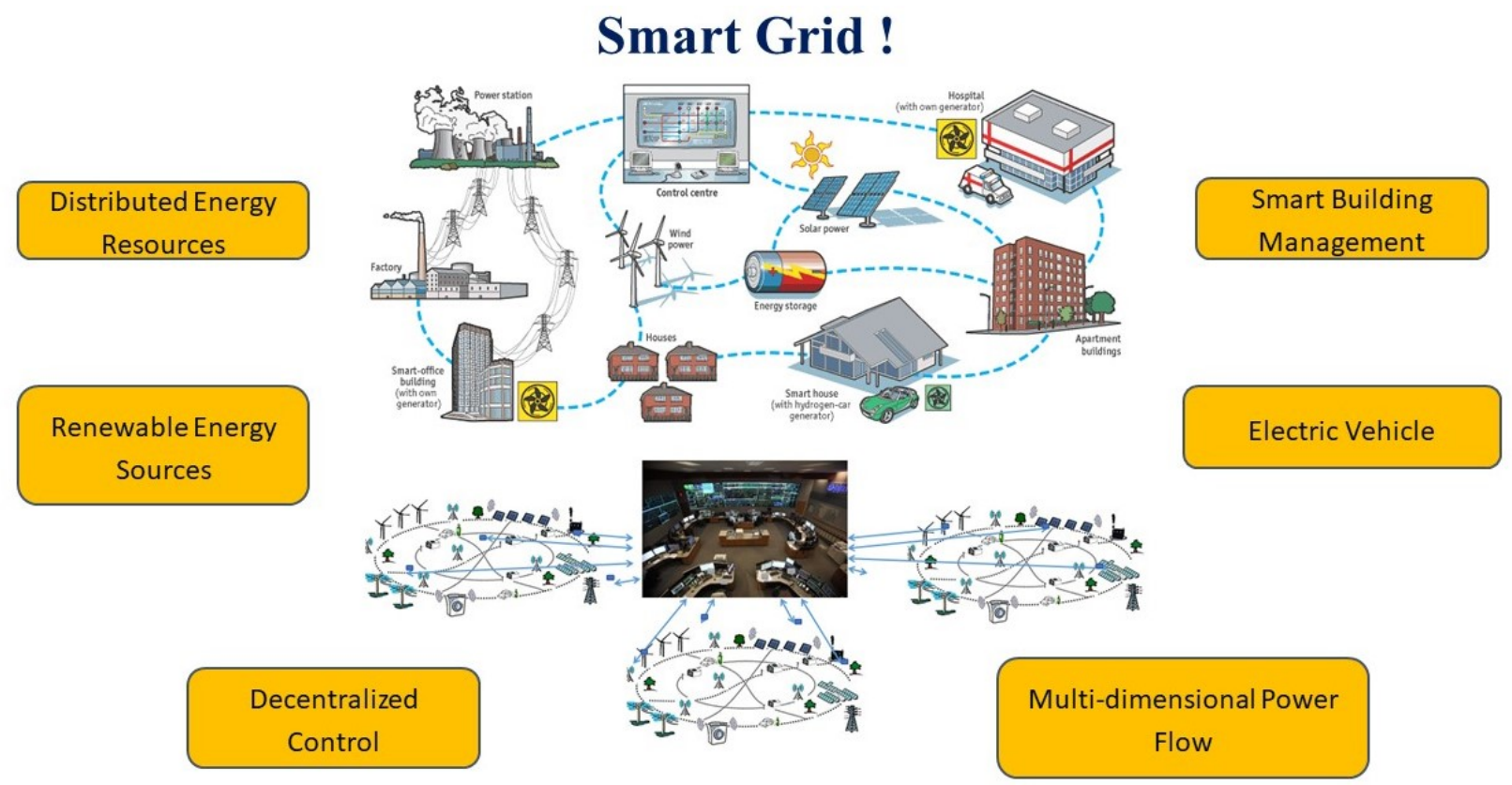

Fig. 1.1 Smart Grid and Components

information like 1) what is the charging starting time 2) what is the charging end time 3) what is the state of charge ( $\mathrm{SoC}$ ) when vehicle enters the charging station 4) what is the $\mathrm{SoC}$ when vehicle departs from charging post 5) the earliest time when vehicle can start charging. Based on these points, the control center decides the charging profile for each vehicle at each charging post. Also vehicle has the option to choose $\mathrm{V} 2 \mathrm{G}$ at the charging post.

The charging of EV is done by household outlets or public charging system. Mode 1 slow AC charging is the most common charging option using the standard household outlets, suitable for small vehicles. Mode 2 also includes household charging but added with an extra protection circuit. Whereas mode 3 is the fast charging method for PHEVs, found in big residential apartments and commercial buildings. Renewable energy sources can be used for charging PHEVs.

As the integration of EV in the grid is increasing, the challenges to optimally charge the EV becomes more prominent. According to the International Energy Agency, number of EVs on road may reach upto 19 million by 2030 in USA alone [7]. Charging in a random way or Uncoordinated/uncontrolled charging can affect the load factor and create load variance, higher voltage drops, and power losses. However, an optimal charging strategy [8]-[9] can minimize the power losses, reduce the load factor and voltage deviation.

Optimal dispatch of DER can also be very challenging. With the deeper penetration of DERs, the uncertainties and imbalance in the grid arise because of inconsistent availability of renewable energy sources, random nature of electric vehicles, and lack of awareness for DR programs. This can lead to uncertainty in load fulfillment in the distribution system. The optimized scheduling 
of DERs can help prevent these conditions. Minimizing the cost of charging while finding the charging and discharging schedule for EVs is another issue. EV owner might not always want to follow the optimized charging profile for their vehicle so the aggregator has to provide some kind of incentive to avoid peak load due to uncoordinated charging.

Load flow analysis is the most important study performed by the utility, required in all stages of the power system. It is performed to compute the steady-state operating values of node voltages, line currents, angles, and power losses at a given load. A power utility needs to analyze these variables at a regular interval to plan for the future in case of some hypothetical critical conditions like system failure or fault analysis. With the DERs and EVs involvement into the grid, there is constant need to perform load flow analysis because of the two-dimensional power flow. For example, the optimal charging strategy for EVs considering Time of Use pricing or Real-Time pricing may not be viable with the network, which can be checked by performing load flow with constraints. This applies to optimized dispatching of DER to help the grid in peak hours. Distribution network constraints need to under limit always. For such reasons, power flow studies are very important. There are different methods for performing load flow for transmission systems in the grid. However, these methods can not be used for distribution system power flow. The utility may not always share the exact network due to security and privacy issues.

To provide the solutions of above problems, we have made the following contributions:

- Chapter 2 presents the coordinated charging for EVs to minimize the load variance and hence reduce the power loss. For EV owners and utility both, we have also formulated our problem as a social welfare problem and maximized their interests using primal-dual approach. In the end, we obtain the optimal dispatch for DERs and EV charging station aggregated profile in a community microgrid to minimize cost of charging.

- Chapter 3 discuss the distributed Resource Allocation (DRA) approach for successful incorporation of a large number of Plug-in Electric Vehicles (PEVs) with the power grid and it is implemented using the concept of achieving output consensus.

- Chapter 4 proposes different deep learning techniques to predict the power flow in a three phase unbalanced distribution network. These neural networks can predict the power flow with very low error in the presence of EVs and rooftop solars in the grid. This proposed method is tested on IEEE 4 node, IEEE123 node test cases, and American Electric Power (AEP) feeder model. The results for Radial Basis Function Network (RBFnet), Multi Layer Perceptron (MLP), and Convolutionary NeuralNetwork (CNN) models are discussed in this chapter.

- Chapter 5 concludes the dissertation and discusses the future work. 


\section{CHAPTER 2}

\section{Optimal Scheduling of Electric Vehicles and Load Flow Analysis}

\subsection{Introduction}

In past few years, due to the increased penetration of Renewable Energy Sources (RES) in the grid, Distributed Energy Resources (DERs) have attracted a lot of attention. The economic and environmental benefits are the driving factors behind this surge in DERs which includes renewable and thermal energy sources. Daily scheduling of DERs which plays an important role in energy cost reduction is discussed in this chapter. One of the important player in the DER is electric vehicles $(\mathrm{EV})$. EVs can be of three type mainly, battery electric vehicle (BEV), hybrid electric vehicle (HEV), and plug in hybrid electric vehicle (PHEV). The simultaneous charging of Electric Vehicles (EVs) put extra burden on the distribution grid. Load congestion, voltage unbalance, voltage stability and overloading of the transformers are some of the issues that need to be managed during charging and discharging of the PHEVs. In this chapter, we discuss the optimal charging scheduling for DERs which consist an electric vehicle charging station (EV-CS) and a battery energy storage system (BESS) in a community microgrid, considering the depth of discharge and other operational constraints. Smart charging optimization is performed for BESS and EVs in the charging station aiming to participate in peak shaving. Also, Coordinated charging for load variance reduction is proposed to mitigate some of the issues mentioned earlier which provides the optimal charging schedule as well for PHEVs. Amidst the talk of such optimal scheduling of EVs, the grid operator or utility also need to ensure the EV owner participation. This brings the EV owner and utility to act together to maximize their profits without affecting the grid from load congestion, voltage instability, and transformer overloading. We formulate the benefits of utility and EV owner as a social welfare problem where we maximize the problem taking into account the generation costs and the battery charging costs. The optimization problem is formulated as a dynamics problem using primal-dual approach. The simulation results are presented to demonstrate the feasibility of the proposed solution and the optimal energy management during the peak load for Time of Use (ToU) pricing. 


\subsection{Literature Survey}

The global carbon emission is contributed mainly by electricity and transportation sector, approximately $65 \%$ of the total carbon dioxide $(\mathrm{CO} 2)$ in the world [10]. The rising concerns of global warming, scarcity of fuel, and gas price hike, popularity of electric vehicles is growing day by day. With the continuous economic growth, the surge in power demand remain one of the crucial problems in the power grid now a days. The modern power grid need to be able to fulfill peak load demand for more time slots which increases the cost and global warming due to the dependence on non renewable sources.

Microgrid is gaining the attention to overcome such problem. A microgrid is a cluster of distributed energy resources (DERs) and interconnected loads which act together as a single entity. Microgrid can operate with connecting to power grid or as stand alone isolated entity. DER consist of distributed generation, battery energy storage system, demand response technologies, plays an important role in curtailing peak load, providing ancialliary services to the microgrid. The advancement of smart grid, integration of renewable energy sources to the grid, electric vehicles and charging station, all help the grid to perform demand side management (DSM) which eventually reduce the operating cost, power losses, peak shaving, control the global warming and many more advantages. With the deeper penetration of DERs, the uncertainties and imbalance in the grid arise because of inconsistent availability of renewable energy sources, random nature of electric vehicles, and lack of awareness for DR programs. This can lead to uncertainty in load fulfillment in the distribution system. The optimized scheduling of DERs can help prevent these conditions. In [11], the author used the particle swarm optimization to optimally schedule the operation duration for the DERs which maximizes the overall benefits of the customers. [12] formulates the DER coordinated scheduling problem in a smart building using the mixed integer linear programming (MILP) technique and compare the cost benefit of the end user with and without DER optimization.

Uncoordinated charging of EVs can put distribution grid into risk. Smart/optimal charging and discharging of EVs to help the grid avoiding peak load, voltage regulation, load congestion, transformer overloading, have been discussed by many researchers in the past [13, 14]. [15-17] discussed the optimal charging optimization in a smart parking lot tied with PV, wind, and diesel RES using Genetic algorithm. The EVs coming to parking lot can supply their energy back to the station for some incentive by enrolling in DR programs. This phenomenon of EVs working to help the grid during the peak load duration is known as Vehicle to grid (V2G). [18] points out that DR can be used as a tool for load shaping during peak load scenario with the EVs. The PHEVs can be charged through household or public charging system. Mode 1 slow AC charging is the most common charging option using standard household outlets, suitable for small vehicles. Mode 2 also includes household charging but added with a extra protection circuit. whereas mode 3 is the 
fast charging method for PHEVs, usually available in big residential and commercial buildings.

Studies shows that Dynamic programming [19, 20], mixed integer linear programming [8, 21], stochastic programming [9],[22],[23], model predictive control [24] have been used for PHEV coordinated charging. Previous research shows that there have been extensive work done towards optimized parking lot allocation in the network [25]. Considering uncertainty of wind power resources, unit commitment is discussed in [18] to schedule the load and PHEVs to reduce charging cost [26]. [27] discusses the peak load reduction using the time of use price for controlled charging of PHEVs. Demand response (DR) also plays very vital role in smart/coordinated charging [28]. Apart from being used as a load, PHEVs can be very useful as a storage during the peak load scenario. This feature when PHEV helps the grid by giving back the energy to the grid while maintaining the minimum required charging for the vehicle, is known as Vehicle to grid (V2G) technology. To enable V2G feature, parking lots will need advanced infrastructure for communication and real time data sharing with the grid [29]. There have been immense research done for optimal planning and allocation of parking lots in a distribution system, optimizing the capacity and location [30], [16]. In [15] the optimal sizing and installation of parking lot are done without considering V2G framework minimizing power loss and voltage regulation. With the V2G mode, it is safe to consider PHEV as load or energy provider.

Most of the previous researchers have used the centralized mode for smart charging of PHEVs which involves the aggregator or control center. This aggregator performs the necessary optimization and send the optimized schedule to charging station considering their profit. However, centralized approach is not well suited for large scale problem considering the communication congestion caused due to high number of vehicles. To solve this problem, researchers have moved towards the distributed/decentralized smart charging and demand control. Decentralized optimization techniques have been used for cost minimization, optimal scheduling of PHEVs, and energy management in power grid. [31],[13] discussed decentralized control for optimal power allocation. They used Karush-Kuhn-Tucker (KKT) conditions of optimality for distributed charging algorithm but the energy management algorithm considered only generation side and leaving out demand side. [32] discussed the social welfare maximization problem for household load management considering flexible loads and PHEV. [33] performed such online welfare maximization by solving the evaluation function of PHEV fleet and minimizing the cost incurred during charging. They defer the online charging requests if the vehicle owners are high in number at a particular time slot. [34] talked about the use of distributed primal-dual algorithm for utility maximization considering real time pricing. However, they do not consider the PHEV.

BESS is another important factor in maintaining the load-generation balance and voltage stability of the system. With the advancement of BESS technologies, the energy storage option is becoming more popular solution for the microgrid. [35] used the simulated annealing method to 
formulate the optimized scheduling problem for BESS for maximum energy arbitrage revenue. Same problem is solved using the dynamic programming to optimizes battery sizing and cost minimization in [36]. These studies have not considered the charging station nor different types of DERs.

In this chapter, we minimize the load variance by optimally charging the EVs. Considering the utility and EV owners benefits, we maximize the social welfare problem using primal dual approach. After getting the coordinated charging schedule of EVs, we find the optimal scheduling for DERs like BESS, distributed generators (DGs), and EV charging station.

\subsection{Charging Coordination of Aggregated Electrical Vehicles for Load Variance Minimization in Distribution System}

Minimizing the load variance ultimately maximizes the load factor of the distribution grid and minimize the power losses. This can also be called as peak load shaving or load levelling techniques.

\subsubsection{Problem Formulation}

In this section, we have considered PHEVs instead of EV as we have considered the everyday distance for travel limitation as 40 miles. With EVs such distance would not need every day charging. However, for the remaining parts of this dissertation, we have considered the status of state of charge (SoC) of the battery for charging. Hence PHEV is replaced by EVs for the sake of uniformity.

PHEVs ability to store the energy and provide to the grid when it needs most, has become very important feature. This is known as vehicle to grid (V2G), which is possible with the advance communication between the grid and PHEV, some necessary hardware installations at the charging station. V2G helps the smart grid in peak shaving and maintaining the stability for example. The objective of the optimization problem is to reduce the load variance. The load variance is minimized for 24 hours.

Whole 24 hours is divided into $\mathrm{T}$ number of slots. The objective function to reduce load variance is formulated as: [37]

$$
\begin{gathered}
\min \sum_{t=1}^{T}\left[\frac{1}{T}\left(\sum_{n=1}^{N} C h_{t, n}+H_{t}-M_{t}^{h}\right)^{2}\right] \\
H_{t}+\sum_{n=1}^{N} C h_{t, n} \leq H_{t, \max },(t=[1 \sim T])
\end{gathered}
$$


subjected to

$$
\begin{aligned}
& -C h_{n, \min } \leq C h_{t, n} \leq C h_{n, \max },\left(t=\left[S_{s} \sim S_{e}\right] ; n=[1 \sim N]\right) \\
& S o C_{n, \min } \leq S o C_{t, n} \leq S o C_{n, \max }, \\
& \left(t=\left[S_{s} \sim S_{e}\right] ; n=[1 \sim N]\right) \\
& M_{t}^{h}=\sum_{t=1}^{T}\left[\frac{1}{T}\left(H_{t}+\sum_{n=1}^{N} C h_{t, n}\right)\right] \\
& \underbrace{s g n_{t, n}}_{\left(t=\left[S_{s} \sim S_{e}\right] ; n=[1 \sim N]\right)}= \begin{cases}1, & C h_{t, n} \geq 0 . \\
-1, & C h_{t, n}<0 .\end{cases} \\
& \underbrace{S o C_{t, n}}_{n=[1 \sim N]}= \begin{cases}\frac{B C_{s}+C h_{t, n} \Delta t}{K_{n}}, & t=S_{s} \\
S O C_{t-1, n}+\frac{C h_{t, n} \Delta t}{K_{n}}, & t=S_{s}+1, \ldots S_{e}\end{cases}
\end{aligned}
$$

The above optimization problem can be formulated to standard quadratic form

$$
\min \left(\frac{1}{2} X^{T} H X+f^{T} X\right)
$$

where $\mathrm{H}=$ block diagonal matrix of size $\mathrm{nT} \times \mathrm{nT}$ and $f=\frac{2}{T}\left(H_{t}-M_{t}^{h}[I]_{1 \times n T}\right)$. Also other equality and inequality constraints were obtained from constraints.

Quadratic Programming has been used to solve this optimization problem where equation 2.22.8 are the constraints.

\subsubsection{Load Flow Analysis}

Load flow analysis is important to compute the voltage regulation, power losses, voltages, and currents in an electrical system. With the increasing penetration of EVs in the grid, urgency to determine line capacity, reliability, unbalance have risen up. Load flow analysis gives the steady state operating conditions. An efficient load flow should be able to give solution to the distribution system of thousands of nodes. In this work, we used backward forward sweep methodology to perform the load flow analysis, which is one of the deterministic load flow methods. This method starts with the end voltages which are assumed to calculate node voltages and branch currents in the network. Difference of this calculated voltages and the rated source voltage should be in limits. Else forward sweep comes into play where node voltages are computed using the rated voltage and 
previously obtained branch currents [38].

\subsection{Optimal Scheduling of EV Charging Station and DERs including BESS in the Community Microgrid}

The goal is to perform the optimal scheduling problem for different types of DERs, scheduling for BESS with the presence of charging station. We not only aim to find the dispatch/discharging schedules of DERs, BESS, and EVs but also the charging schedules of BESS and charging station are optimized. We consider a community microgrid where we assume to have two natural gas fired generators and two diesel fired generators as DERs, a BESS, and a charging station which help to reduce the minimizing the operational cost. The optimization problem is formulated as a simple linear programming (LP) problem and trying to solve it with the MATLAB LP solver. To check the viability of the proposed framework, we also perform a load flow analysis to find the voltage profile.

\subsubsection{System Model}

We consider a community microgrid which has various components like distributed generators (DGs), BESS, and EV charging station. Figure 2.1 [39] shows the the schematic diagram of community microgrid considered in this work. In this section, we will present the mathematical model of each entity.

\subsubsection{Battery Energy Storage System Model}

Battery energy storage system (BESS) has emerged as an important technique to improve the energy management in the power grid due to the evolution of batteries of high storage capacity [40, 41]. In order to model optimization problem for charging discharging scheduling, we need to understand the functioning of BESS operation. BESS can store the energy and discharge it as whenever grid needs it. BESS play an important role in demand fulfillment and increases the system efficiency by matching the demand-generation. BESS can make system more reliable by helping the grid to tackle the unavailability and uncertainty of renewable energy sources. Functioning of BESS depends on state of charge, temperature, and depth of discharge. we have ignored the effect of temperature on BESS in this work. The energy output of BESS is computed by the energy stored in two continuous instances [42] and is given by

$$
E_{t+1}^{B E S S}=E_{t}^{B E S S}+P_{j t, c h}^{B E S S} \eta_{c h} \Delta t
$$




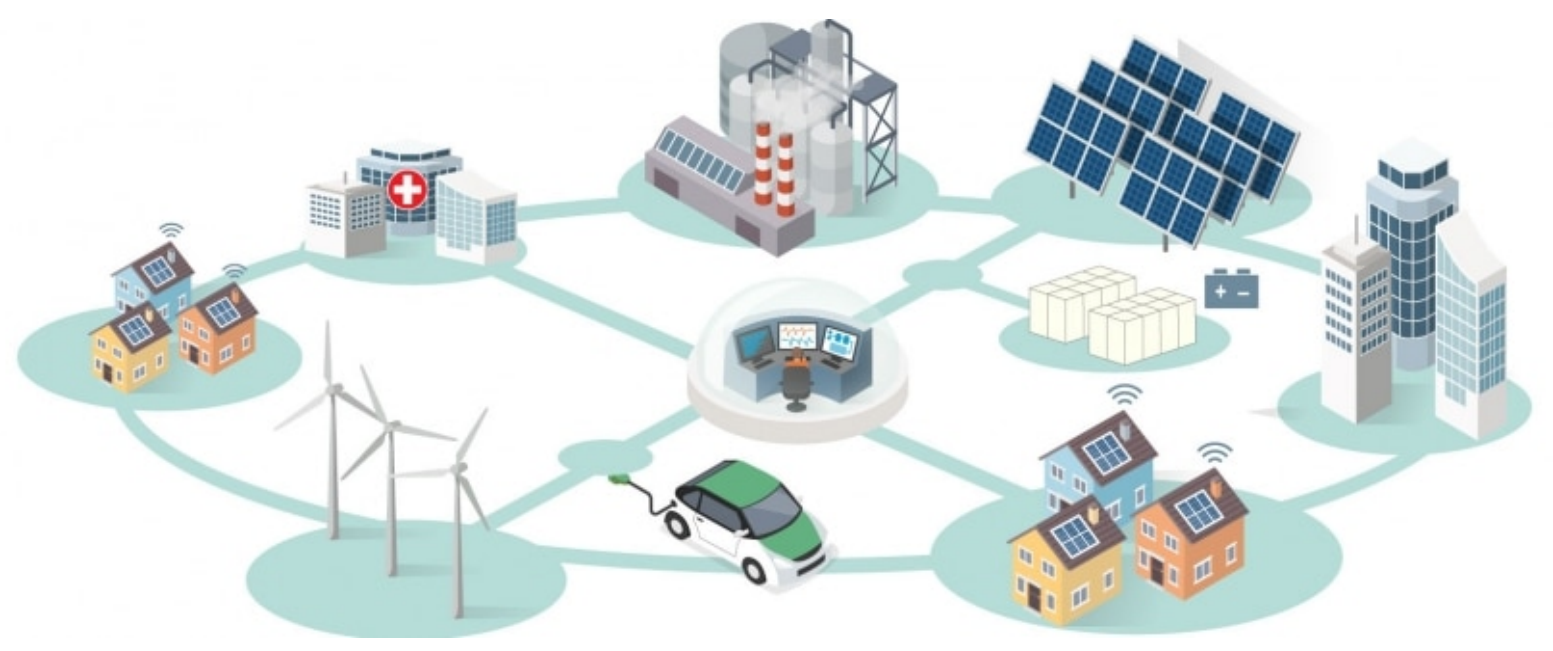

Fig. 2.1 Community Microgrid

$$
E_{t+1}^{B E S S}=E_{t}^{B E S S}-\frac{P_{j t, d i s}^{B E S S}}{\eta_{d i s}} \Delta t
$$

where $\eta_{c h}$ and $\eta_{d i s}$ are efficiency of charging and discharging of BESS respectively and $j \in$ $[1, \mathrm{~B}]$ where $B$ is the number of BESS in the microgrid. When the battery is charging, $P_{b t, c h}^{B E S}$ will be negative, acting as a load while in case of discharging, $P_{b t, d i s}^{B E S S}$ will be positive and act as a generator. The Another important constraint in BESS is the limit on state of charge (SoC) of the battery [28], which is given by

$$
\begin{gathered}
S o C_{j \min }^{B E S S} \leq \frac{\eta_{c h} P_{j t, c h}^{B E S S} T}{C_{B}} \leq S o C_{j \max }^{B E S S} \\
S o C_{j \min }^{B E S S} \leq \frac{P_{j t, d i s}^{B E S S} T}{\eta_{d i s} C_{B}} \leq S o C_{j \max }^{B E S S}
\end{gathered}
$$

$\mathrm{T}$ is the sample time and $C_{B}$ is the rated energy capacity of battery. Equation 2.11 and 2.12 represents the SoC range for both charging and discharging. In [43], author has considered two cases for the operational constraints on SoC. The normal case keep the SoC between 30-65 \% while for emergency case SoC is assumed to be $20-80 \%$. However in this work, we are considering only emergency case scenario.

$$
\sum_{t=1}^{24} P_{j t, d i s}^{B E S S} \leq D_{o} D_{j}
$$

The Depth of Discharge (DoD) is defined as the percentage of battery is drained with respect to it's capacity. Equation 2.13 represent the constraint on DoD limit. 
The constraint on energy store in BESS is given by

$$
E_{\min }^{B E S S} \leq E_{j t}^{B E S S} \leq E_{\max }^{B E S S}
$$

\subsubsection{Electric Vehicle Charging Station}

A large scale BESS need a high installation and operational cost. In compare to that EVs can be an economical way to support grid during peak pricing. When charging EV, it appear as a load.EVs can be very helpful in providing support to the grid during the peak load scenario by giving their stored energy back to the grid. This process is called as Vehicle to Grid (V2G) operation. We considered a charging station for electric vehicles in the community microgrid which was not included in author's previous work [28]. [42] considered only grid to vehicle mode of operation for optimal scheduling of DERs. In this work, we have considered V2G scenario. It is ensured that all EVs leave with their needs being met when they leave the charging station. so the constraints for EVs at charging station would be mainly due to the factor of EV battery SoC and charging power.

$$
S o C_{r \min } \leq S o C_{r} \leq S o C_{r \max }
$$

where $r \in[1, V], V$ represents the number of vehicles. Some other constraints based on EV battery energy capacity, battery energy balance are described as [44]

$$
\begin{gathered}
E_{r, t+1}^{E V}=E_{r, t}^{E V}+\eta_{c h}^{E V} P_{r, t}^{E V} \Delta t \\
E_{r, t+1}^{E V}=E_{r, t}^{E V}-\frac{P_{r, t}^{E V} \Delta t}{\eta_{d i s}^{E V}}
\end{gathered}
$$

$\Delta t$ here represent the optimization time interval and assumed to be 1 hour.

\subsubsection{Distributed Energy Resources}

The role of distributed energy resources is vital in helping the grid to fulfill load needs in the system. It makes the grid more reliable and provide ancillary services to grid even during the peak load scenarios. DERs allow the customers to reduce cost of energy and eventually increases the profit as with DERs, customers can sell their electricity back to grid and be a part of various demand response strategies. DERs include mainly distributed generators, EVs, energy storage system, rooftop PV, wind turbines, demand side management, smart meters with two way flow of information making the grid smarter. While dealing with DERs in the system, it is important to know the hosting capacity; what is the correct amount of DER to which system can function within it's operating/technical limits. 


$$
P_{i}^{\text {min }} \leq P_{i t} \leq P_{i}^{\max }
$$

equation 2.18 formulates the operational constraint on output power from $i_{t h}$ DER at $t$ hour.

\subsubsection{Optimization problem}

The main objective is to find optimal schedule for DERs including BESS and EV charging and discharging schedule. We try to find the optimum dispatch information about the DERs. The objective function is formulated as Linear programming problem in which we minimize the cost incurred in operating the DERs, charging station, BESS, and the load supplied by the grid. The optimization ensures that the DERs dispatched to meet the given system load is the most economical option available and reduces the cost for each time interval of one hour during the 24 hours time horizon of optimization duration. The cost function for the system is given by

$$
\begin{aligned}
\min \left(J_{t}\right)= & \sum_{t=1}^{24}\left(\sum_{i=1}^{N}\left(c_{i} P_{i t}\right)+\sum_{j=1}^{B}\left(c_{j} P_{j t, d i s}^{B E S S}\right)+\left(C_{t} P_{G t}\right)-\sum_{j=1}^{B}\left(C_{t} P_{j t, c h}^{B E S S}\right)\right) \\
& +\sum_{r \in d}\left(c_{r} P_{r, t}^{E V}\right)-\sum_{r \in s}\left(c_{r} P_{r, t}^{E V}\right)
\end{aligned}
$$

The charging station operation is divided into two sets of time duration, $d$ shows the time when EVs are discharging and acting as a load and $s$ contains the charging hours for EVs. During $s$ hours, charging station act as a load for grid and do not help the grid to match demand generation gap. so the active power balance in the system is given by

1. when $t \in d$

$$
P_{G t}+\sum_{i=1}^{N} P_{i t}+\sum_{j=1}^{B} P_{j t, d i s}^{B E S S}+P_{r t}^{E V}=P_{d t}
$$

2. when $t \in s$

$$
P_{G t}+\sum_{i=1}^{N} P_{i t}+\sum_{j=1}^{B} P_{j t, d i s}^{B E S S}=P_{d t}+P_{r t}^{E V}
$$

\subsection{Social Welfare problem for Electric Vehicle Charging}

We formulated social welfare problem of EV charging as primal dual, convex optimization problem. We also illustrated the effect of ToU pricing on the charging demand with and without energy management. The load flow analysis is performed to check the viability of the mentioned approach on the IEEE 34 node test case. 


\subsubsection{EV Charging Model}

The state of charge (SoC) of battery is defined as "the percentage of the maximum possible charge that is present inside a rechargeable battery". There are several models of SoC being discussed by the researchers considering the different objective functions for optimal charge scheduling of EVs. We have considered the simplified charging model [45] for battery of $i^{\text {th }} \mathrm{EV}$ as

$$
y_{i}(k+1)=y_{i}(k)+\frac{\alpha_{i}}{B_{i}} x_{i}
$$

where $y_{i}(k)$ is the State of Charge (SoC) of the $i^{t h}$ vehicle at $k^{t h}$ time step and $x_{i}$ is the charging demand or charging power $(\mathrm{kW})$ of $i^{t h}$ vehicle. $\alpha_{i}$ denotes the charger efficiency and $B_{i}$ is the battery size of $i^{\text {th }}$ vehicle. The charger efficiency is assumed to be constant. It is evident from the equation 2.22 that for the higher charging efficiency and/or higher charging demand, the time taken to charge the battery is less. For $k \in[0, T]$, if $y_{i}(T)=1$, this implies that each battery is fully charged.

\subsubsection{Primal Dual Gradient}

The primal dual gradient is used to find the primal and dual solution of an inequality constrained convex or concave optimization problem. In this part, we are solving the convex optimization problem through primal dual gradient method. Consider the constrained optimization problem [46]

$$
\begin{array}{cl}
\underset{x_{i} \in \mathbb{R}^{n}}{\operatorname{minimize}} & f(x) \\
\text { subject to } & A x=b \\
& h_{i}(x) \leq 0, i=1, \ldots . m
\end{array}
$$

for $A \in \mathbb{R}^{p \times n}, x \in \mathbb{R}^{p}$. here $f(x)$ is a strictly convex and $h_{i}(x)$ is a convex function. The optimal solution to problem 2.23 is $x^{*}$ (the primal dual optimizer) if and only if there exists $\lambda^{*} \in$ $\mathbb{R}^{p}, \mu^{*} \in \mathbb{R}^{m}$ such that the following Karush-Kuhn-Tucker (KKT) conditions are satisfied

$$
\begin{aligned}
& \nabla_{x} f\left(x^{*}\right)+\sum_{i=1}^{m} \mu_{i} \nabla_{x} h_{i}\left(x^{*}\right)+A^{T} \lambda_{i}^{*}=0 \\
& h_{i}\left(x^{*}\right) \leq 0, \mu_{i}^{*} h_{i}^{*}(x)=0
\end{aligned}
$$

Apply primal dual gradient method to KKT conditions to get the dynamics. Solution to KKT conditions are the solution of 2.23. So we get the following dynamics 


$$
\begin{aligned}
& -\tau_{x} \dot{x}=\nabla_{x} f(x)+\sum_{i=1}^{m} \mu_{i} \nabla_{x} h_{i}(x)+A^{T} \lambda_{i} \\
& \tau_{\mu_{i}} \dot{\mu_{i}}=h_{i}(x) \\
& \tau_{\lambda_{i}} \dot{\lambda_{i}}=A x-b
\end{aligned}
$$

where $\tau_{x}, \tau_{\lambda}, \tau_{\mu}$ are positive definite matrices. The above dynamical system 2.25 converges to the optimal solution $x^{*}$ [47]. A version of the proof is reported in [48].

\subsubsection{Problem Formulation}

The objective is to maximize the social welfare problem considering the operational constraints. The social welfare problem is formulated as a concave $\left(U_{i}\left(x_{i}\right)\right)$ - convex $(U(\bar{x}))$ function to maximize the profits of vehicle owner and utility. The social welfare problem [49] is formulated as

$$
\begin{array}{ll}
\underset{x_{i}, \bar{x}}{\operatorname{maximize}} & \sum_{i=1}^{N}\left(U_{i}\left(x_{i}\right)-U(\bar{x})\right) \\
\text { subject to } & \bar{x}=\sum_{i=1}^{N} x_{i} \\
& x_{i}^{\text {min }} \leq x_{i} \leq x_{i}^{\text {max }} .
\end{array}
$$

Where,

$$
U_{i}\left(y_{i}\right)=b_{i}-\gamma_{i}\left[y_{i}(k+1)-y_{i}(k)\right]^{2}
$$

is modeled as a charging cost function of the EVs. $\gamma_{i}>0$ is the trade-off parameter between cost and SoC. and

$$
U(q)=\rho_{1} q^{2}+\rho_{2} q+\rho_{3}
$$

represents the quadratic power allocation formulation for utility. Also, the charging equation is modeled as [45]

$$
y_{i}(k+1)=y_{i}(k)+\frac{\alpha_{i}}{B_{i}} x_{i}
$$

by replacing these values in equation 2.26 , we get

$$
\underbrace{\max }_{y_{i}, q} \sum_{i=1}^{N}\left(\left(b_{i}-\gamma_{i}\left[y_{i}(k+1)-y_{i}(k)\right]^{2}\right)-\left(\rho_{1} q^{2}+\rho_{2} q+\rho_{3}\right)\right)
$$

In order to maximize first component of equation 2.24, we need to minimize the cost of battery charging from vehicle owner's perspective which is this term $\gamma_{i}\left[y_{i}(k+1)-y_{i}(k)\right]^{2}$. Also we try to minimize the allocated power to avoid peak load from utility's perspective which is $\rho_{1} q^{2}+\rho_{2} q+\rho_{3}$. 
For the sake of clarity, we have omitted the index $k$. By further simplification from equation 2.23 and 2.24, we get the objective function as

$$
\underbrace{\max }_{x_{i}, q} \sum_{i=1}^{N}\left(\left(b_{i}-\gamma_{i}\left(\frac{\alpha_{i}}{B_{i}} x_{i}\right)^{2}\right)-\left(\rho_{1} q^{2}+\rho_{2} q+\rho_{3}\right)\right)
$$

the term $\gamma_{i}$ represents the trade-off parameter between the cost of charging and comfort of EV owners. Equation 2.25 can be written as

$$
\begin{array}{ll}
\underset{x_{i}, \bar{x}}{\operatorname{minimize}} & \sum_{i=1}^{N}-\left(\left(b_{i}-\gamma_{i}\left(\frac{\alpha_{i}}{B_{i}} x_{i}\right)^{2}\right)-\left(\rho_{1} q^{2}+\rho_{2} q+\rho_{3}\right)\right) \\
\text { subject to } & q=\sum_{i=1}^{N} x_{i} \\
& x_{i}^{\text {min }} \leq x_{i} \leq x_{i}^{\max } .
\end{array}
$$

Our optimization problem 2.30 is in the structure of constrained optimization problem 2.23. so the Lagrangian of 2.30 is given by

$$
\begin{gathered}
\mathcal{L}=-\left(b_{i}-\gamma_{i}\left(\frac{\alpha_{i}}{B_{i}} x_{i}\right)^{2}\right)+\left(\rho_{1} q^{2}+\rho_{2} q+\rho_{3}\right)+ \\
\lambda\left[\sum_{i=1}^{N} x_{i}-q\right]^{+}+\mu_{i}\left[x_{i}^{\text {min }}-x_{i}\right]^{+}+\nu_{i}\left[x_{i}-x_{i}{ }^{\text {max }}\right]^{+}
\end{gathered}
$$

The primal dual gradient laws given in 2.25 for the convex optimization problem 2.30 are shown below

$$
\begin{gathered}
\dot{x}_{i}=\left(-2 \gamma_{i}\left(\frac{\alpha_{i}}{B_{i}}\right)^{2} x_{i}(k)-\lambda+\mu_{i}-\nu_{i}\right) \\
\dot{q}=\lambda-2 \rho_{1} q-\rho_{2} \\
\dot{\lambda}=\left[\sum_{i=1}^{N} x_{i}-q\right]^{+} \\
\dot{\mu}=\left[x_{i}{ }^{\text {min }}-x_{i}\right]^{+} \\
\dot{\nu}=\left[x_{i}-x_{i}{ }^{\text {max }}\right]^{+}
\end{gathered}
$$

here $\lambda$ is the cost parameter and $\mu_{i}, \nu_{i}$ are the positive definite matrices. Equation 2.30 represents the optimization problem while 2.32-2.36 are the dual functions. 


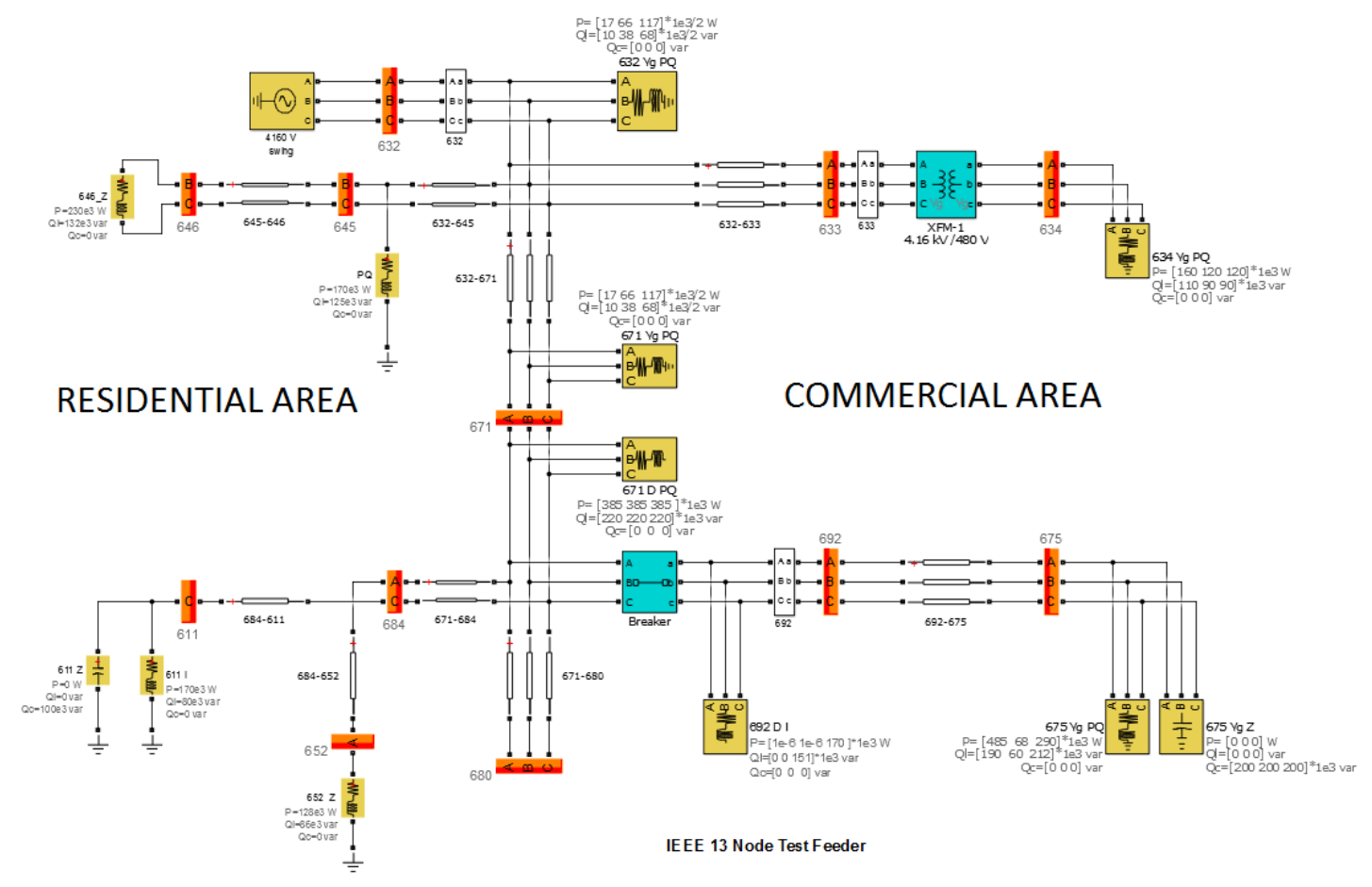

Fig. 2.2 IEEE 13 Node Test System

The primal dual dynamics converge asymptotically to the solution of optimization problem 2.22 .

\subsection{Results}

\subsubsection{Load Variance Minimization}

The optimization problem is applied to test on IEEE 13 node distribution system, as shown in figure 2.2. Table 2.1 shows the system data while load data for the test system is given in table 2.2. In table 2.2 , last column represents the connection type $\mathrm{Y} / \Delta$ and nature of load which could be constant current,voltage or constant load type. The test system is divided into two areas: residential and commercial. The residential area consist of households while commercial area has small schools, small size hotels, stand alone retail shop, and medium offices. The load is applied at node 646 and node 611 in the residential area, while node 634 and 675 is assumed to be in the commercial side. Load profiles for both the areas are found from [50] for Morgantown, West Virginia as shown in figure 2.3. We have assumed the number of PHEVs in the node 646 as 150 and at node 611, there are 115 PHEVs. The commercial area contains 100 PHEVs at 634 and 50 at 675 node. Also the vehicles in the residential area leave their home at 9:00 AM and come back at 5:00 PM while vehicles in industrial or commercial area is supposed to be present 24 hours. 
Table 2.1 System Configuration

\begin{tabular}{|c|c|c|c|}
\hline Node A & Node B & Length (ft) & Configuration \\
\hline 632 & 645 & 500 & 603 \\
\hline 632 & 633 & 500 & 602 \\
\hline 633 & 634 & 0 & XFM-1 \\
\hline 645 & 646 & 300 & 603 \\
\hline 650 & 632 & 2000 & 601 \\
\hline 684 & 652 & 800 & 607 \\
\hline 632 & 671 & 2000 & 601 \\
\hline 671 & 684 & 300 & 604 \\
\hline 671 & 680 & 1000 & 601 \\
\hline 671 & 692 & 0 & Switch \\
\hline 684 & 611 & 300 & 605 \\
\hline 692 & 675 & 500 & 606 \\
\hline
\end{tabular}

Table 2.2 Load Data

\begin{tabular}{|c|c|c|c|c|c|c|c|}
\hline Node & Ph-1 & Ph-1 & Ph-2 & Ph-2 & Ph-3 & Ph-3 & Load \\
\hline 634 & 160 & 110 & 120 & 90 & 120 & 0 & Y-PQ \\
\hline 645 & 0 & 0 & 170 & 125 & 0 & 0 & Y-PQ \\
\hline 646 & 0 & 0 & 230 & 132 & 0 & 0 & D-Z \\
\hline 652 & 128 & 86 & 0 & 0 & 0 & 0 & Y-Z \\
\hline 671 & 385 & 220 & 385 & 220 & 385 & 220 & D-PQ \\
\hline 675 & 485 & 190 & 68 & 60 & 290 & 212 & Y-PQ \\
\hline 692 & 0 & 0 & 0 & 0 & 170 & 151 & D-I \\
\hline 611 & 0 & 0 & 0 & 0 & 170 & 80 & Y-I \\
\hline
\end{tabular}

Lower and upper bound of state of charge for each vehicle is set as 0.2 and 0.8 respectively. The charging efficiency of each PHEV is set as 0.92 . For the sake of simplicity, it is assumed that all PHEVs are of the same ratings. Weibull distribution is used to predict the distance travelled by each vehicle.

\subsubsection{Vehicle to Grid (V2G) Mode}

Under V2G mode, PHEV assists the grid to peak load shaving. Figure 2.3 gives the optimized charging profile for each load in residential and commercial area. 


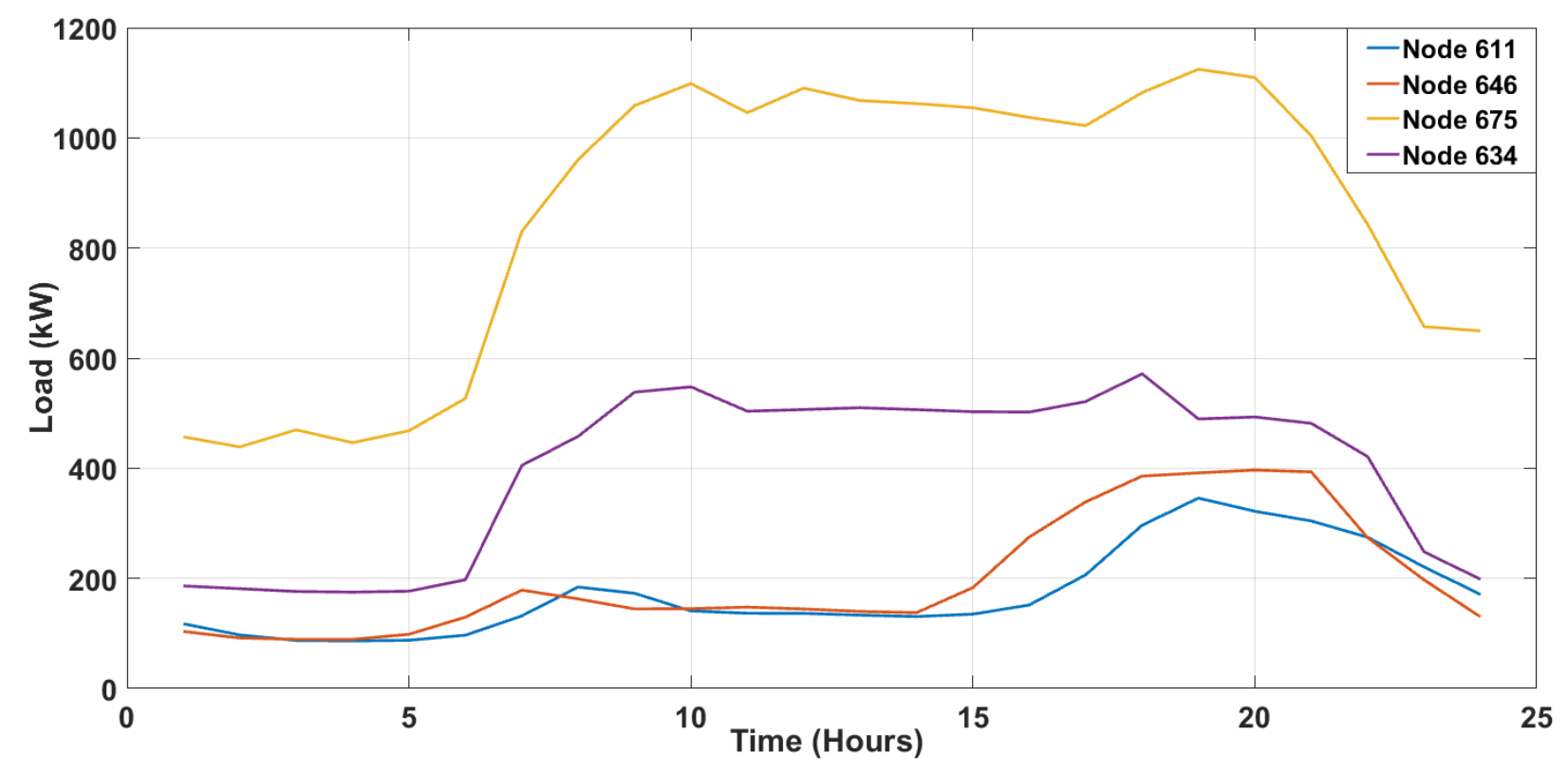

Fig. 2.3 Load Profile
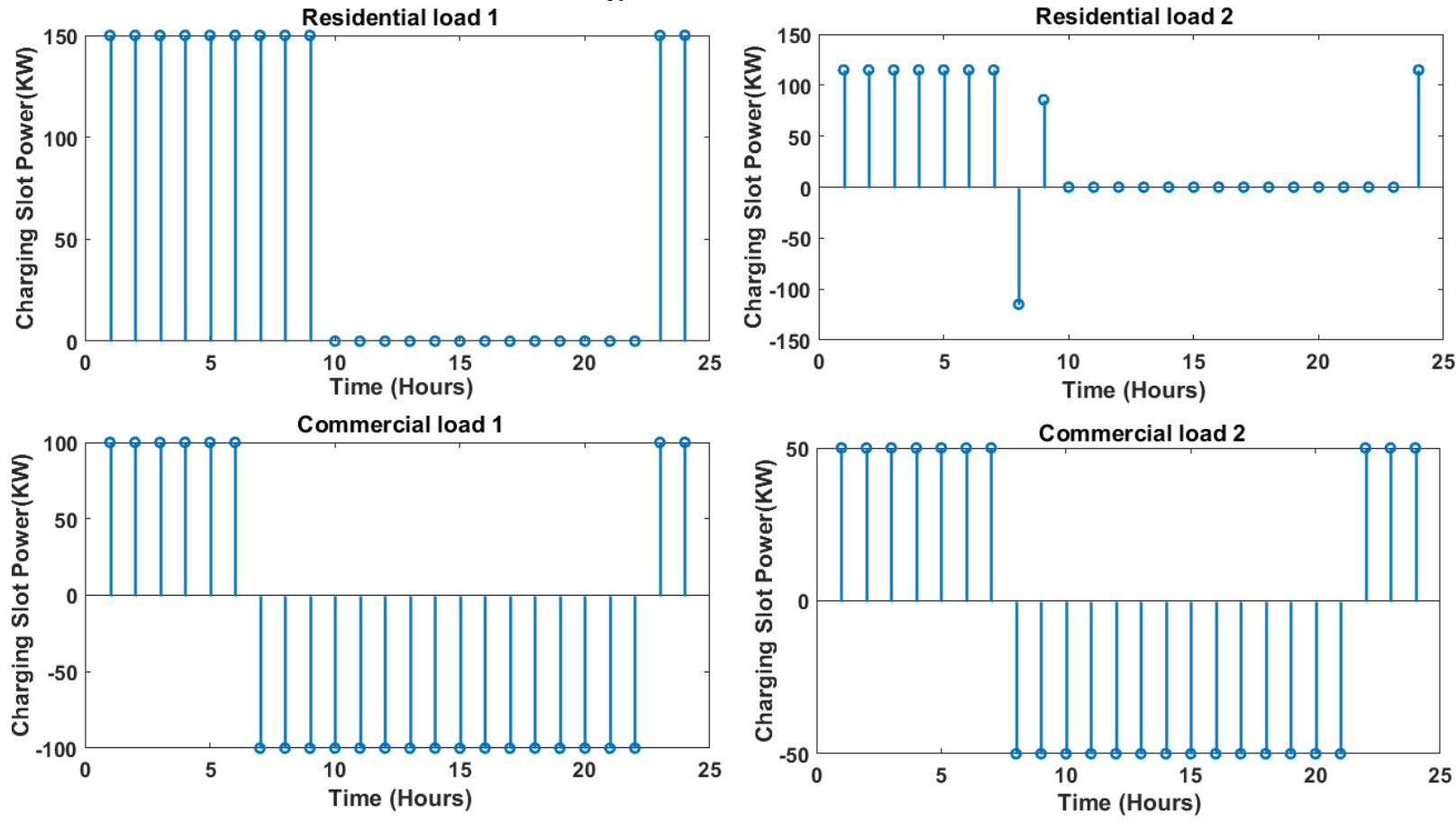

Fig. 2.4 Charging Profile in Vehicle to Grid Mode

As we can see from figure 2.4, almost all PHEVs can be charged until 9 AM from a nearby parking station without creating instability to grid. Also some of the PHEVs are able to give the energy back to the grid for an hour in the day time while for more hours during the evening time when grid needs it mostly because of the peak loads. 

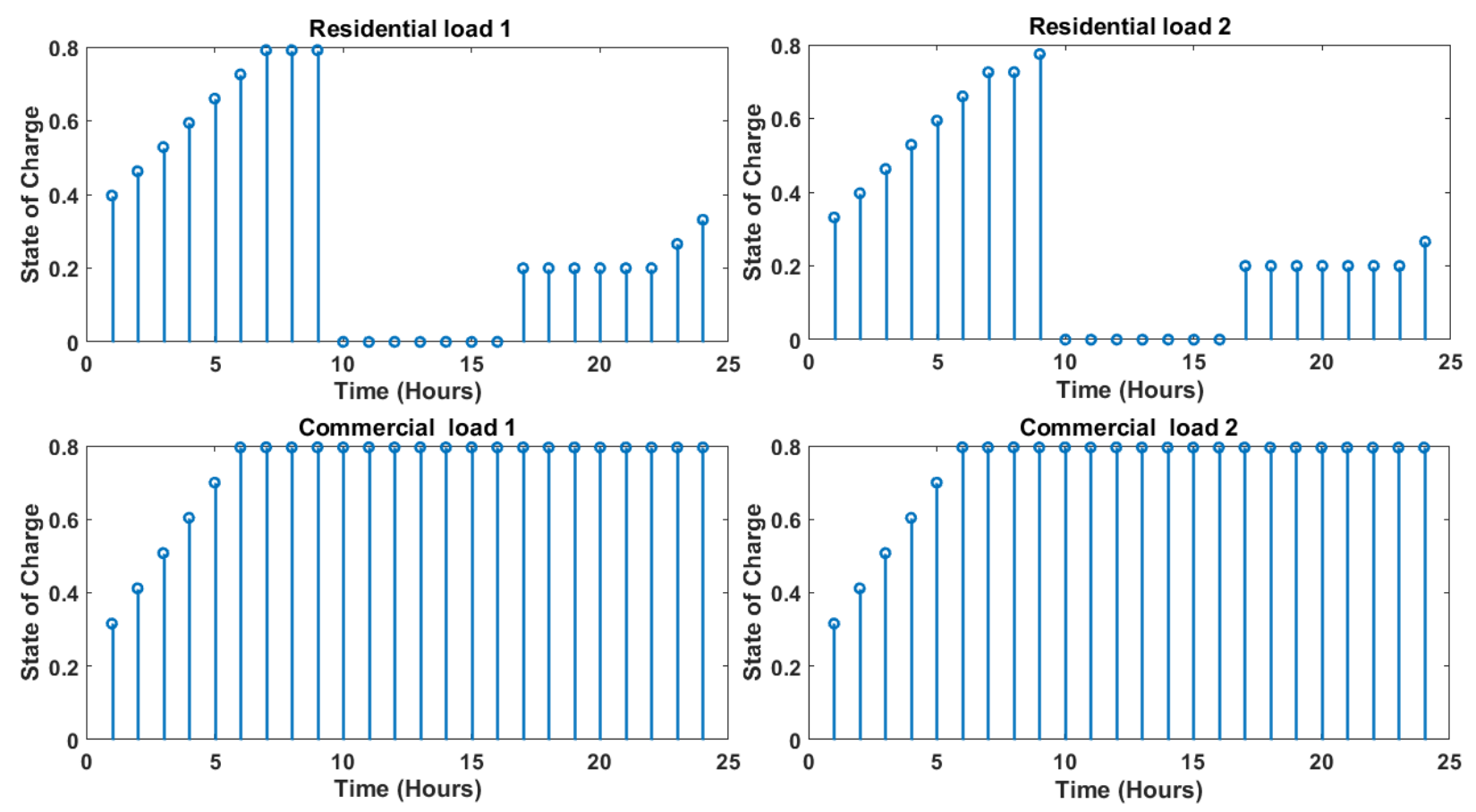

Fig. 2.5 SOC in Vehicle to Grid Mode

Similarly in the commercial side, the charging profile have been shown. Figure 2.5 presents the state of charge curves for all the loads. The load variance minimization for each load is shown in figure 2.8. It is quite evident from the figure 2.8 that load variance is reduced with coordinated charging. Each figure has plots for residential and commercial areas. Table 2.3 represents the voltage unbalance at each node during Vehicle to grid optimization.

\subsubsection{Grid to Vehicle Mode}

This mode does not allow the energy to be flown back to grid. The charging profile and state of charge curve is shown in figure 2.6 and 2.7 respectively. As we can see, during no time PHEVs able to help grid. Figure 2.9 shows the load variance curve for each node. As we can see, load variance is not reduced much even with coordinated charging as compared to V2G mode. 

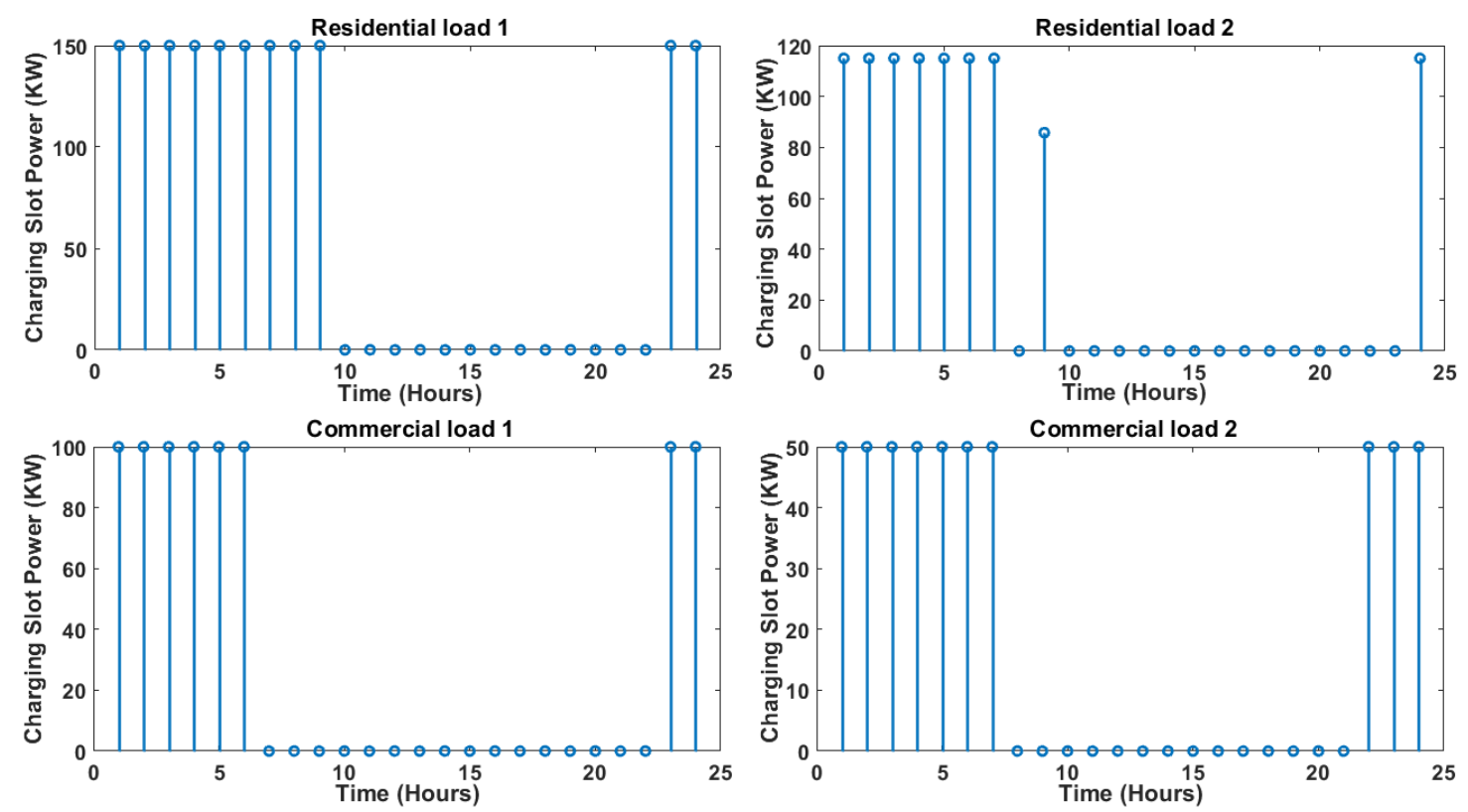

Fig. 2.6 Charging Profile in Grid to Vehicle Mode
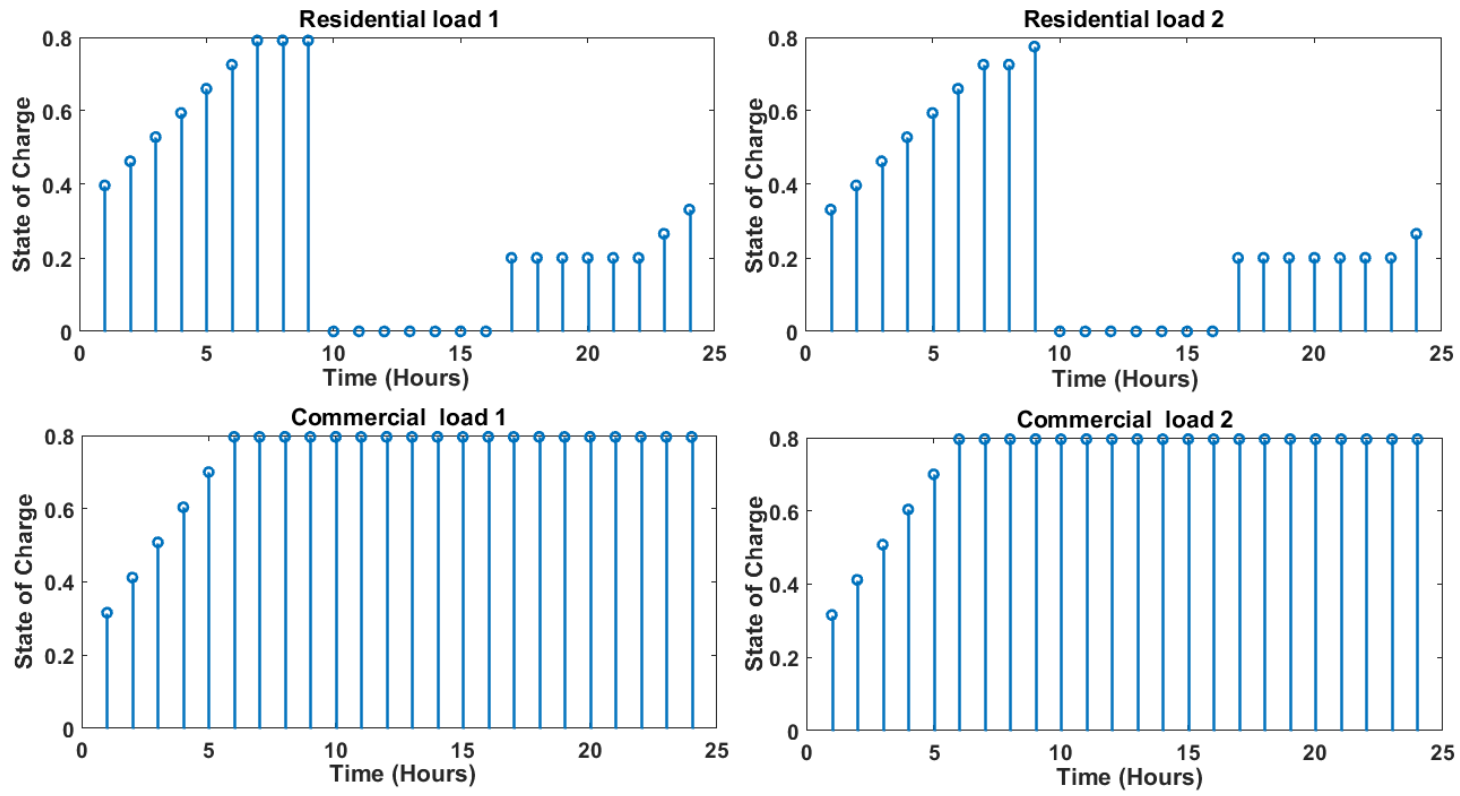

Fig. 2.7 SOC in Grid to Vehicle Mode 


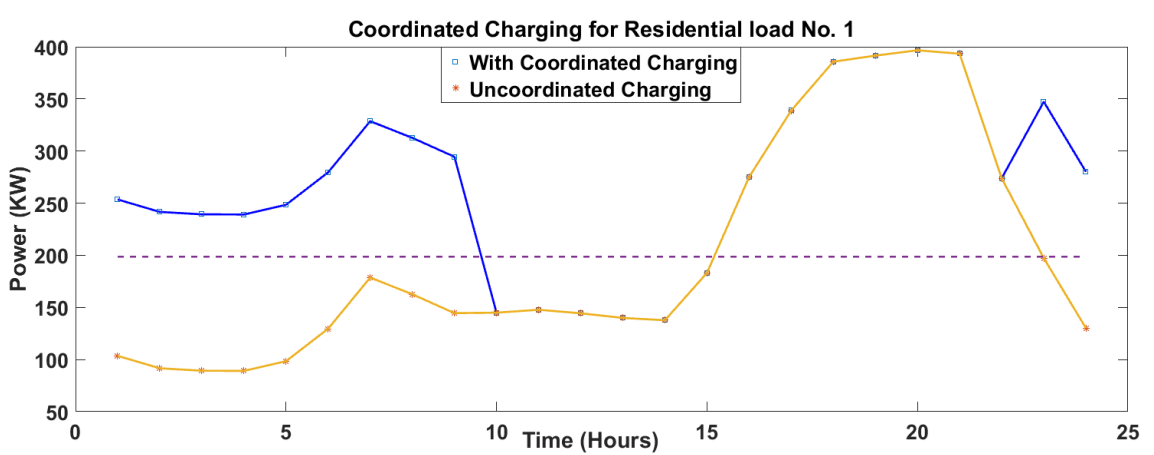

(a)

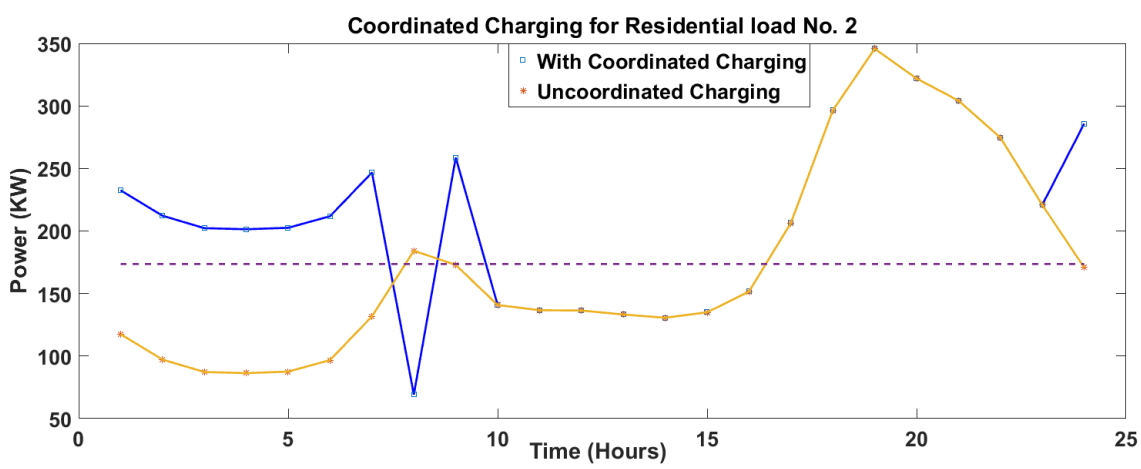

(b)

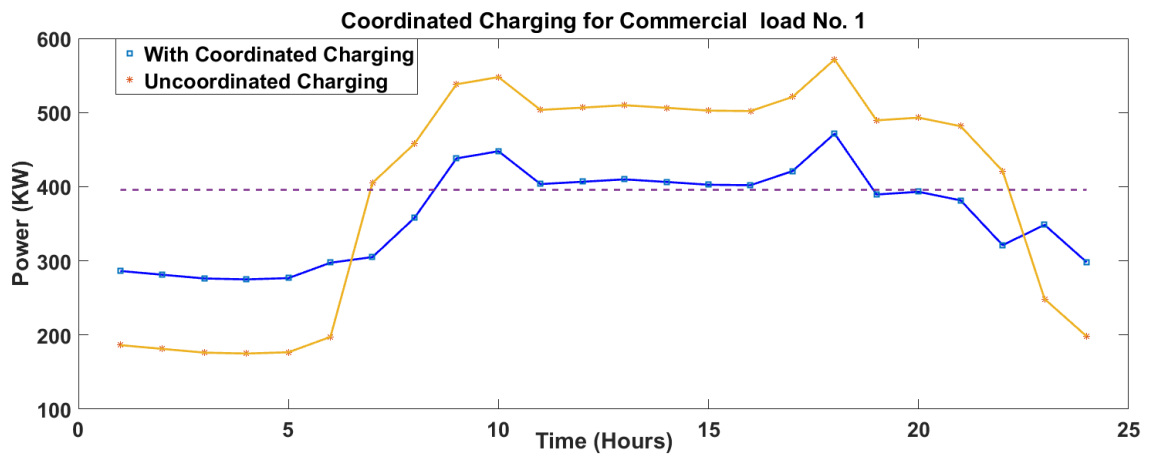

(c)

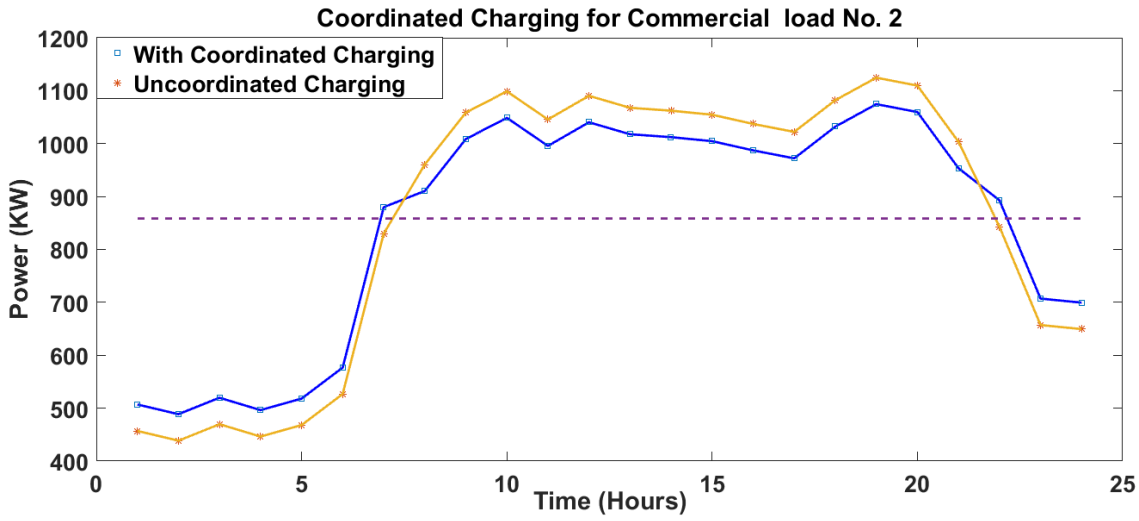

(d)

Fig. 2.8 Load Variance Minimization in Vehicle to Grid Mode at (a) Node 646 (b) Node 611 (c) Node 634 (d) Node 675 


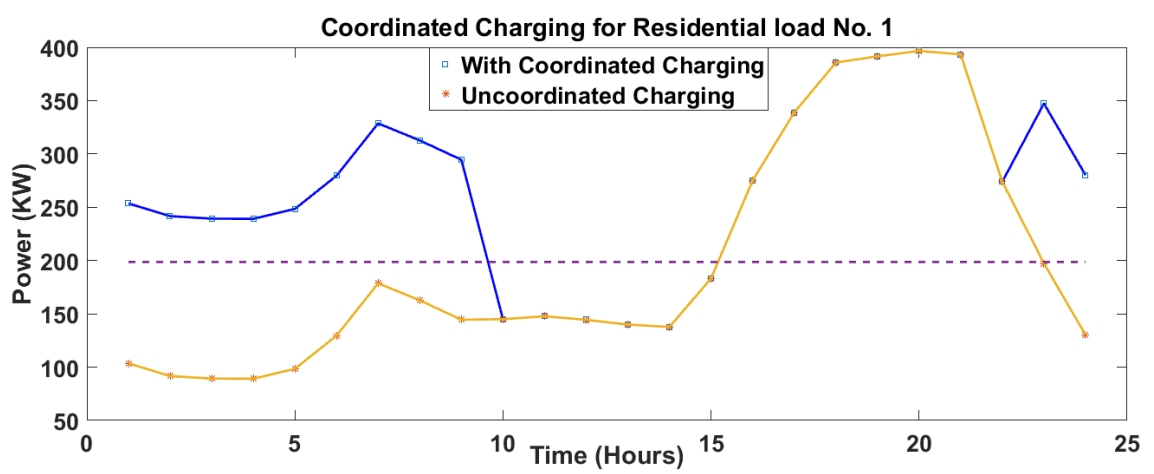

(a)

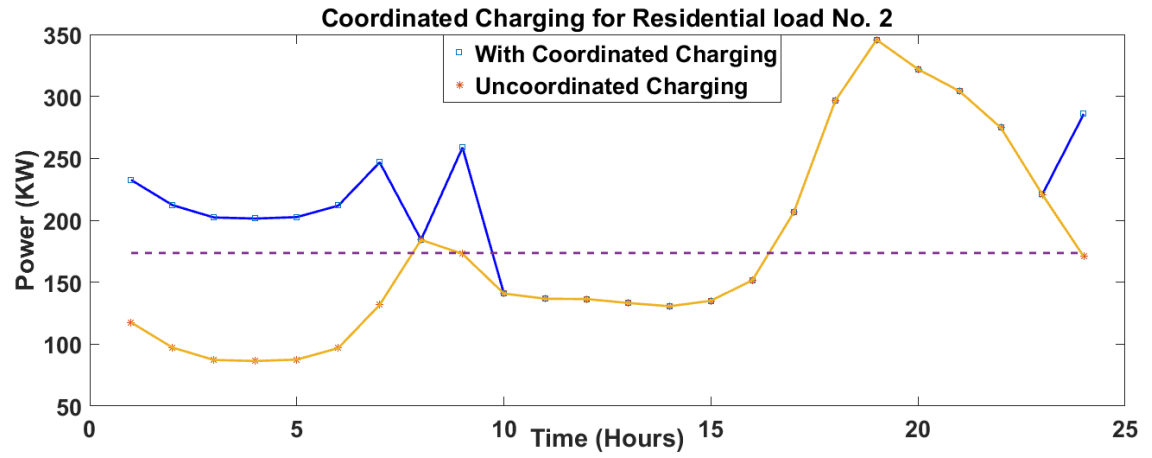

(b)

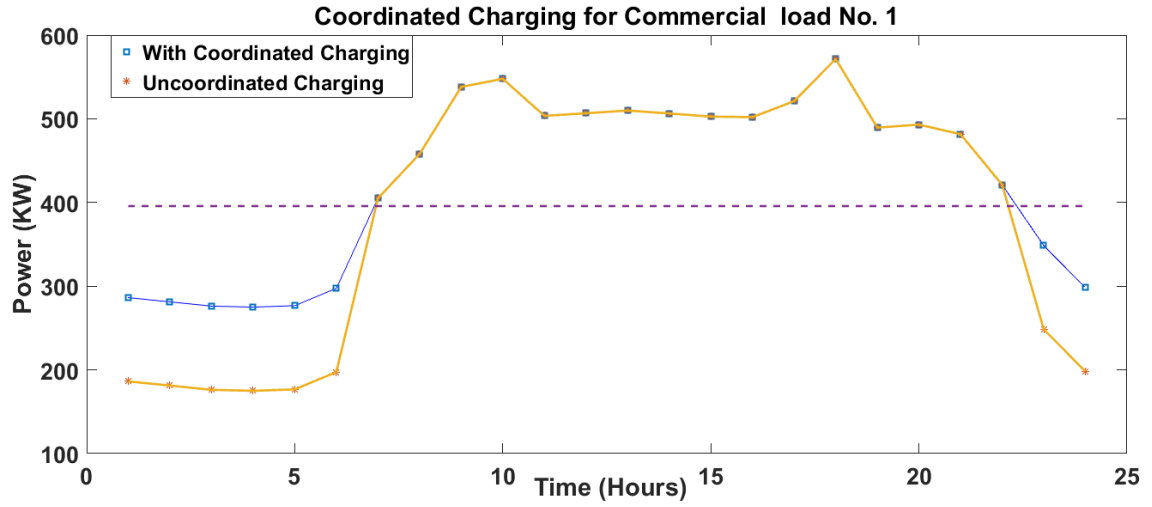

(c)

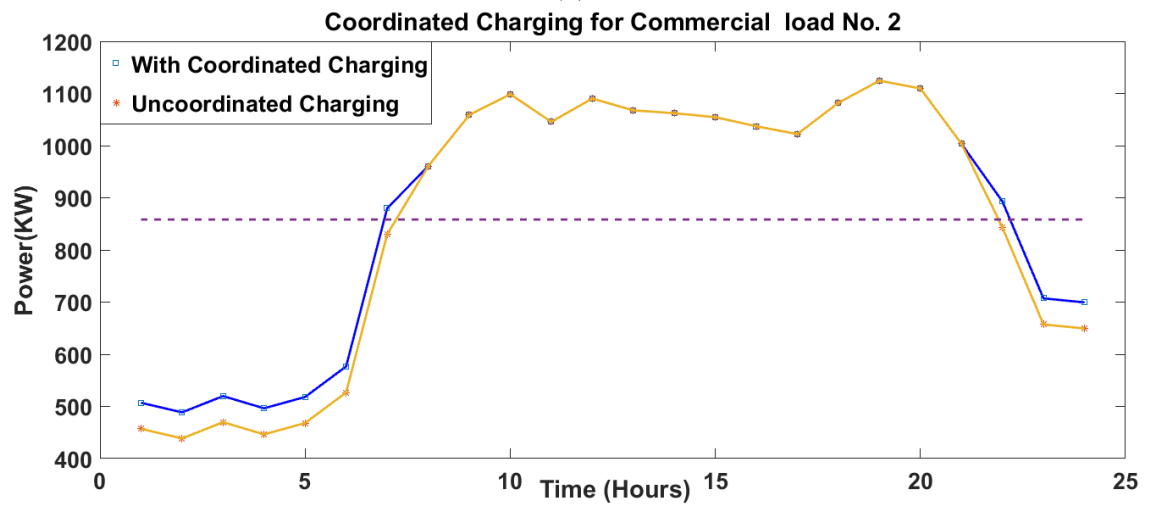

(d)

Fig. 2.9 Load Variance Minimization in Grid to Vehicle Mode at (a) Node 646 (b) Node 611 (c) Node 634 (d) Node 675 
Table 2.3 Voltage Unbalance at All Nodes

\begin{tabular}{|c|c|}
\hline Node & Unbalance in Voltage $\%$ \\
\hline 646 & 0.1744 \\
\hline 611 & 0.1946 \\
\hline 634 & 0.0195 \\
\hline 675 & 0.6113 \\
\hline
\end{tabular}
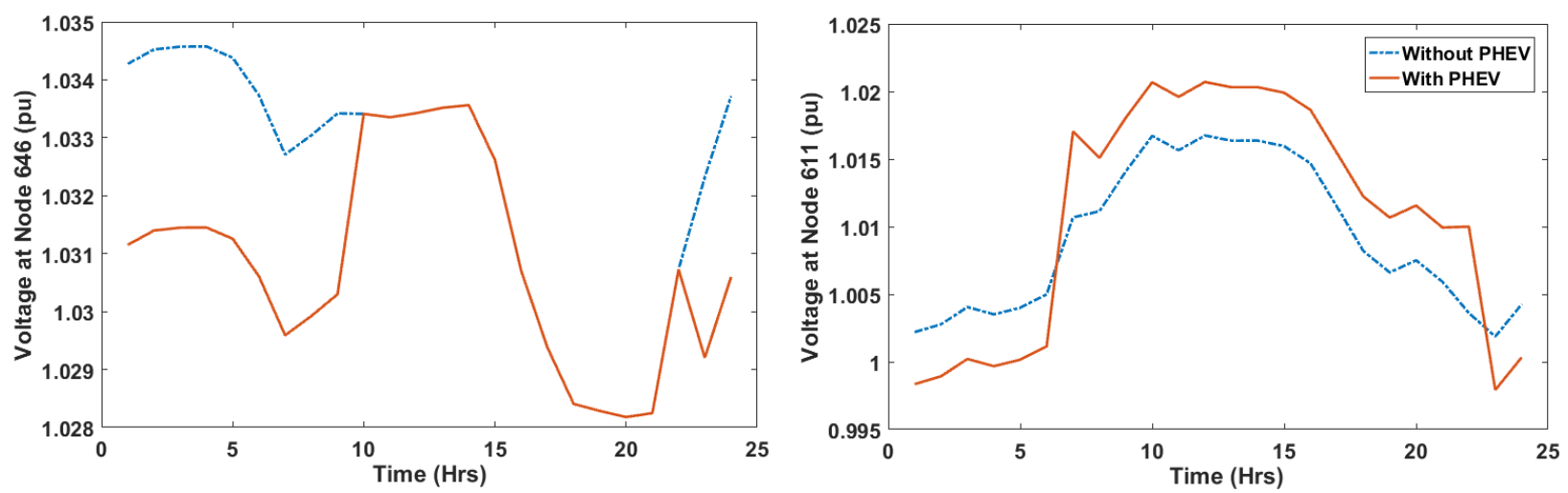

(a)
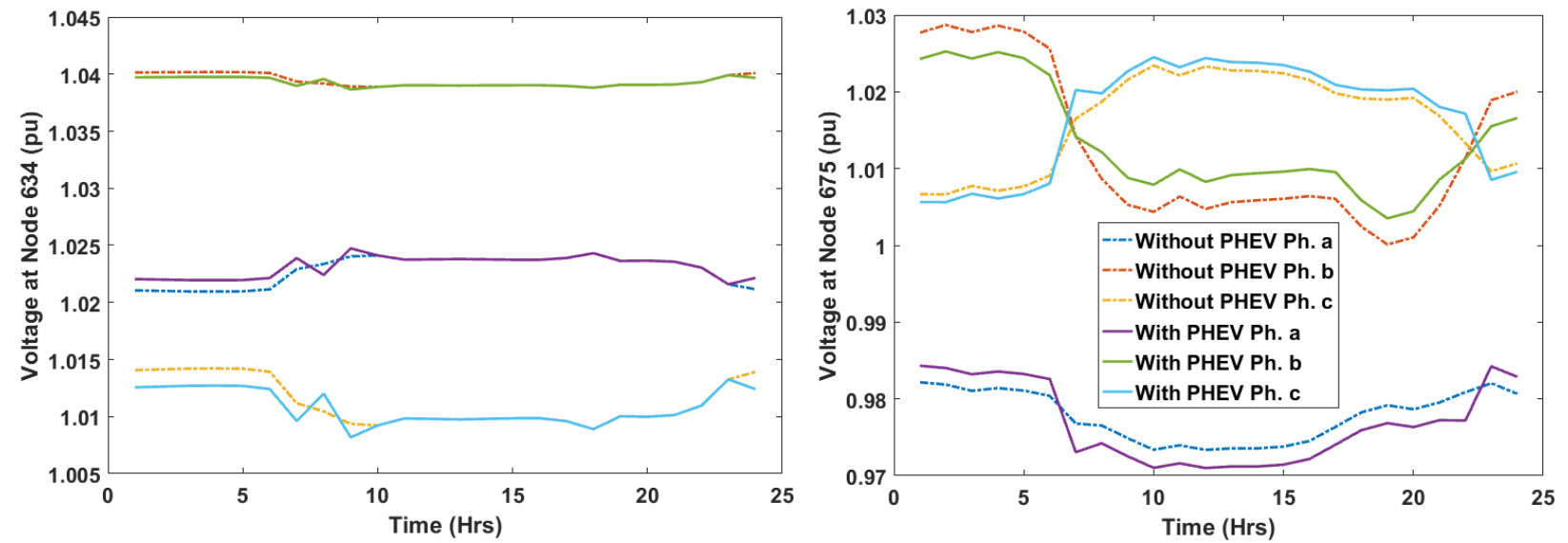

(b)

Fig. 2.10 Voltage during Vehicle to Grid Mode at (a) Node 646 - Node 611 (b) Node 634 - Node 675 


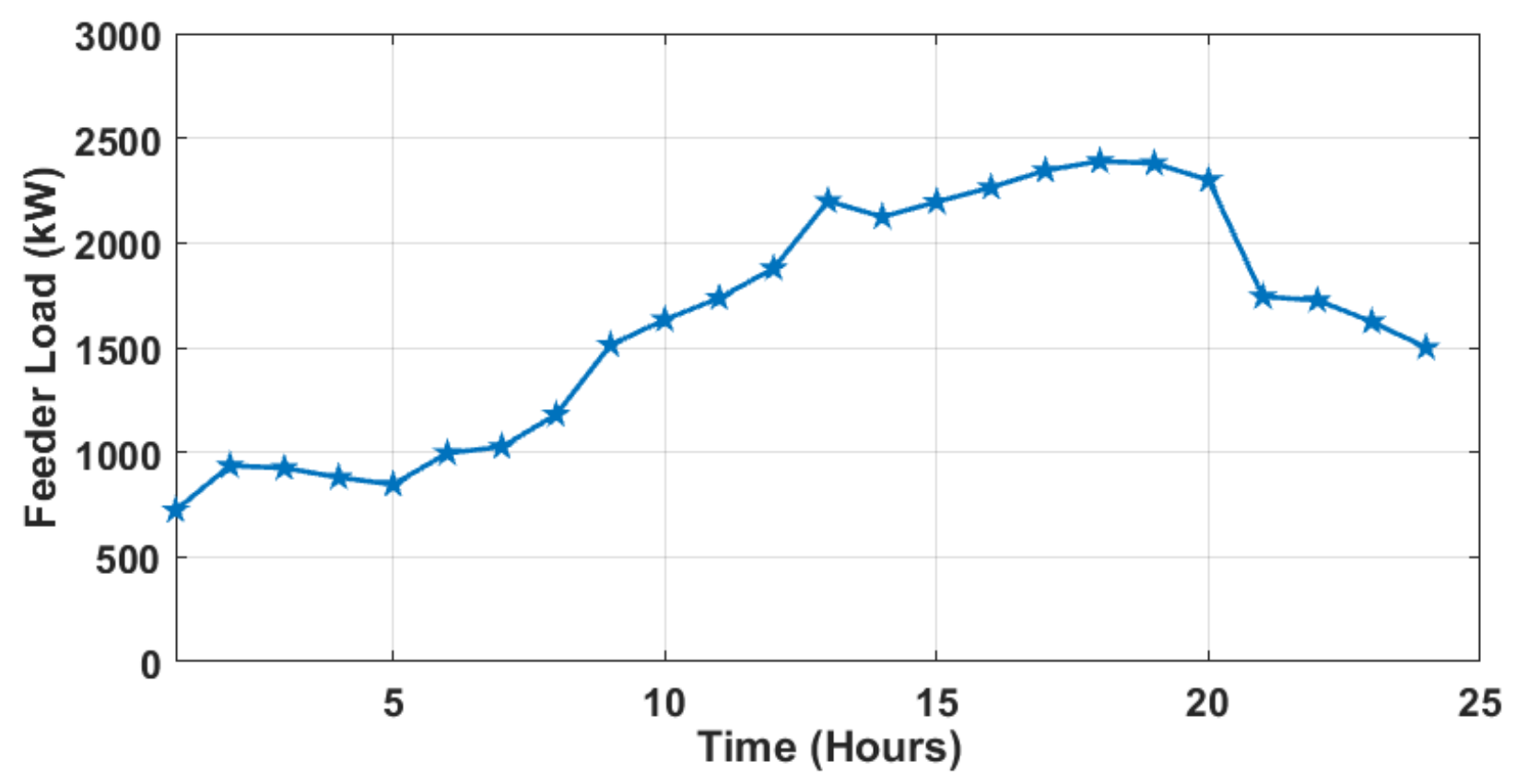

Fig. 2.11 24-hour Feeder Load Profile

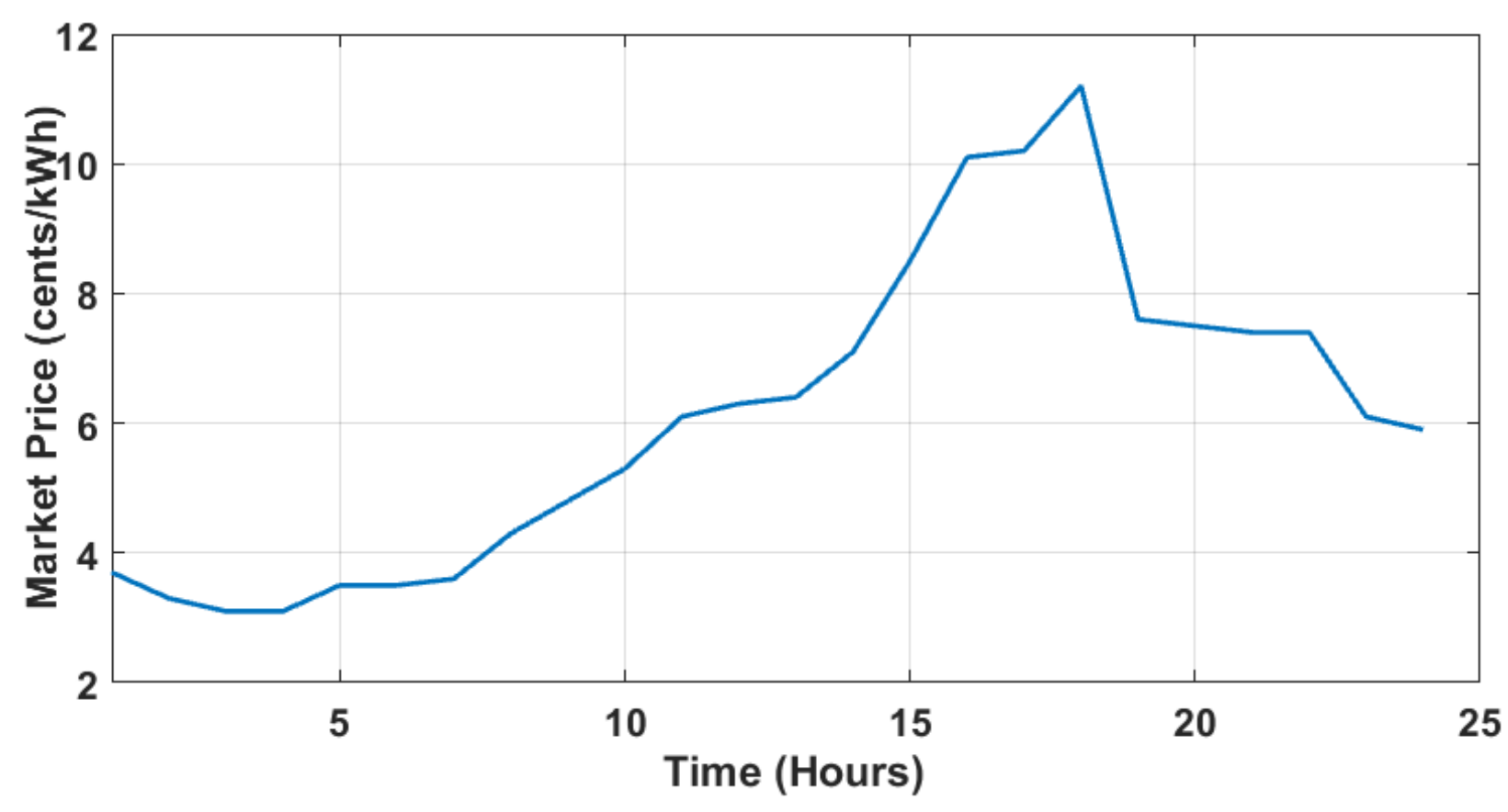

Fig. 2.12 Market Forecast for 24 hours

\subsubsection{Optimal Scheduling of EV-CS and DERs}

We have considered a fictitious microgrid based taxonomy distribution feeder developed by PNNL [51]. The fictitious microgrid is assumed to have two natural gas fired generators, two diesel fired 
Table 2.4 DER Data

\begin{tabular}{|l|l|c|c|c|}
\hline $\begin{array}{l}\text { DER } \\
\text { Name }\end{array}$ & Type & Offers (cents/kWh) & Min kW & Max kW \\
\hline DG1 & $\begin{array}{l}\text { Natural } \\
\text { Gas Fired } \\
\text { Generators }\end{array}$ & 8.4 & 100 & 100 \\
\hline DG2 & $\begin{array}{l}\text { Diesel } \\
\text { Fired } \\
\text { Generators }\end{array}$ & 7.9 & 100 & 100 \\
\hline DG3 & $\begin{array}{l}\text { Natural } \\
\text { Gas Fired } \\
\text { Generators }\end{array}$ & 9.4 & 200 & 200 \\
\hline DG4 & $\begin{array}{l}\text { Natural } \\
\text { Gas Fired } \\
\text { Generators }\end{array}$ & 9.1 & 150 & 150 \\
\hline DG5 & $\begin{array}{l}\text { Diesel } \\
\text { Fired } \\
\text { Generators }\end{array}$ & 8.3 & 200 & 200 \\
\hline RL & $\begin{array}{l}\text { Responsive } \\
\text { Load }\end{array}$ & 15 & 0 & 200 \\
\hline ST & BESS & 12 & 0 & 200 \\
\hline MG & Main Grid & Market Data & 0 & 150 \\
\hline
\end{tabular}

generators, an energy storage unit, a responsive load (RL). In addition to this, an EV charging station is considered as an important entity to construct a community microgrid. The DER cost data and constraints are shown in table 2.4. The load profile is achieved from the dataset developed by NREL [52]. Figure 2.11 shows the aggregated 24 hour load profile of a microgrid, used in our simulations as an input. The other important parameter is electricity prices. We have used a day ahead forecast market price from ComEd utility. The hourly prices for an average summer day are shown in figure 2.12. The objective function is modeled as linear programming model and solved by MATLAB LP solver. Optimum DER dispatch schedule is presented in figure 2.13. As shown in the figure, we have the optimized schedule of when and how much power these 5 DGs and one responsive load are going to supply to grid to help it match the demand considering the operational cost. Each subplot corresponds to the dispatch schedule of each type of DER. When the market forecast price is low, grid does not seek help from any other resources, instead it matches the load by itself. During the peak hours 15-19, DERs come to help the grid following their optimized dispatch schedule. It is quite evident from the figure that DERs and grid together match the system load.

The BESS charging/discharging is shown in figure 2.14. Until 9th hour, BESS battery has 

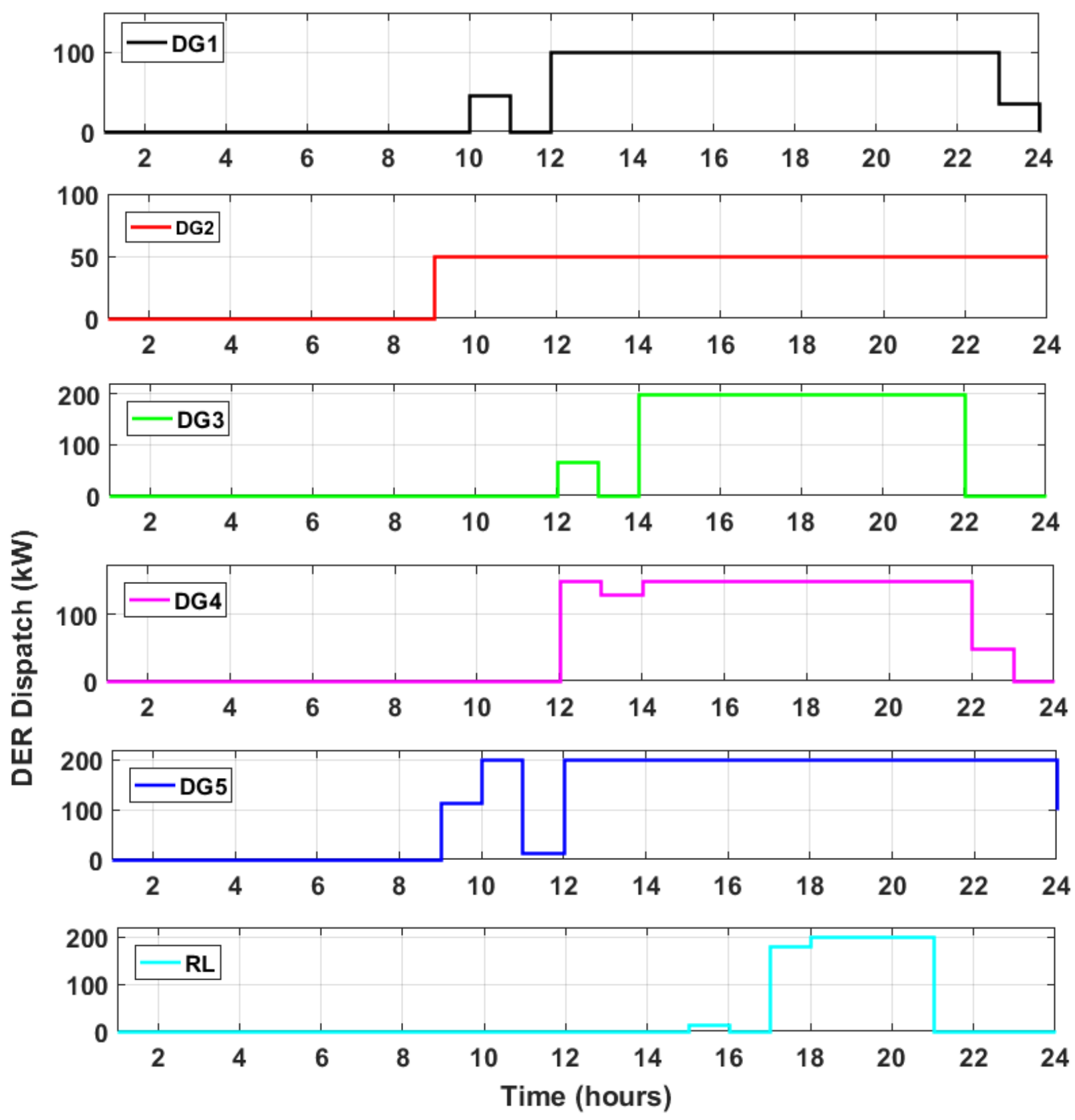

Fig. 2.13 Optimal DER Dispatch Schedule

maintained it's energy constant at $800 \mathrm{~kW}$. During 10-15 hours, BESS continue to discharge amidst the surge in market prices.

In figure 2.15, we have the forecast load profile of EV charging station and the optimized charging schedule. The solid line depicts the forecast load profile for EV. When the charging station has unmet load at a particular hour, we shift the aggregated load demand to next hour which is shown by dotted line in the figure 2.15. This shifting of load is permissible for maximum two hours. As we can see from the plot, EV-CS has load of $220 \mathrm{~kW}$ at 18:00 hours, while the 


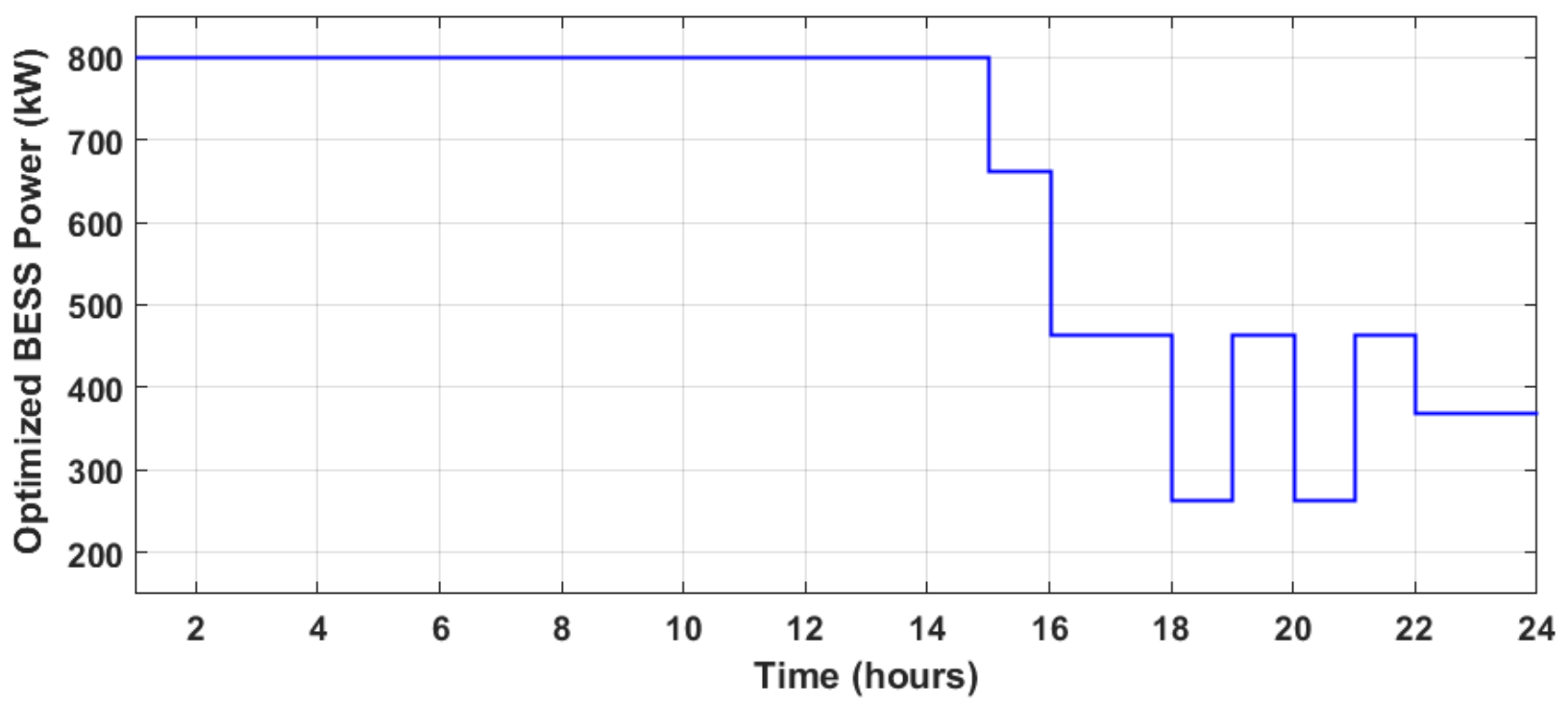

Fig. 2.14 Optimum BESS Charge/Discharge Schedule

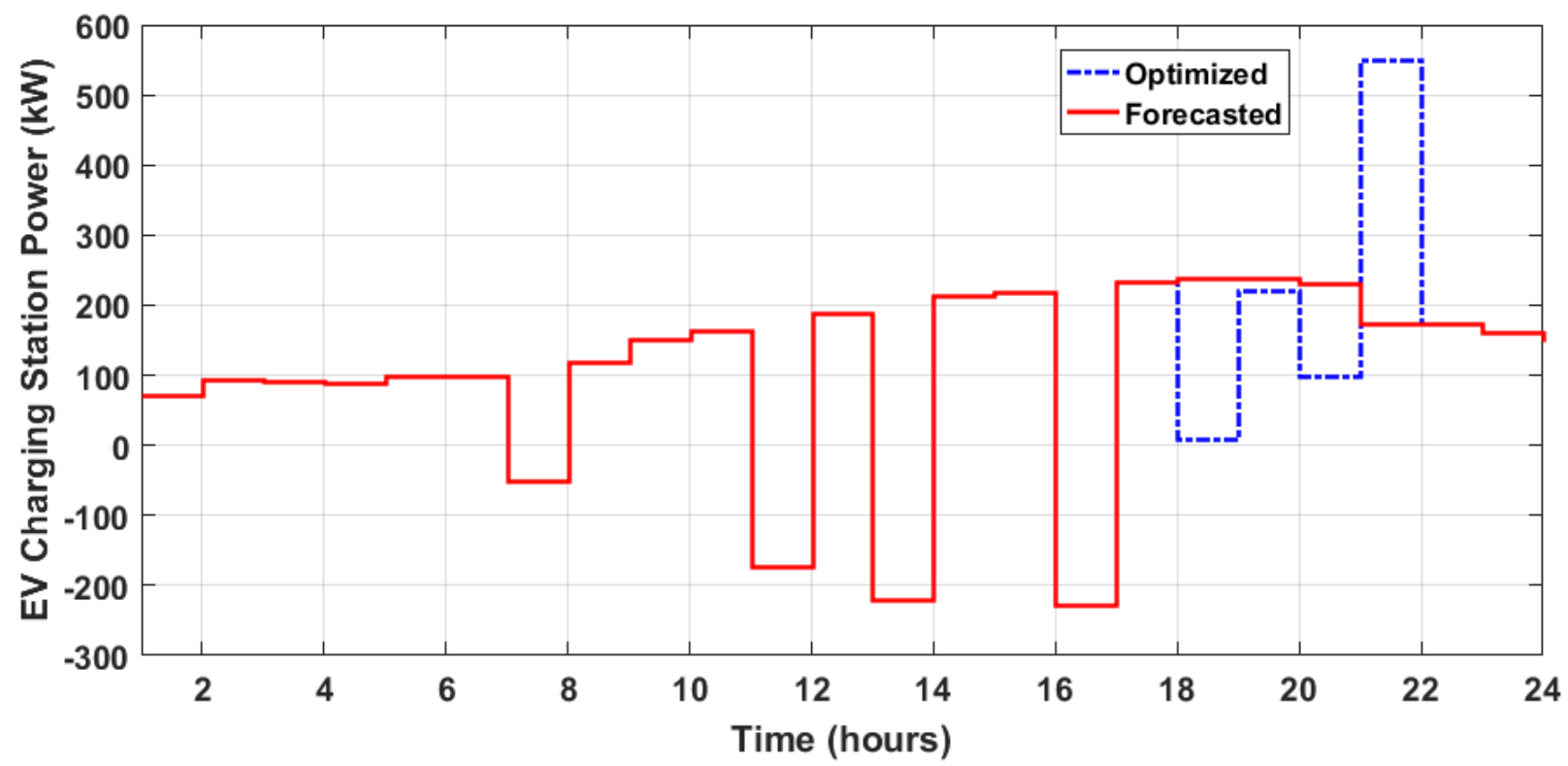

Fig. 2.15 Aggregated Optimal Charge/Discharge Schedule for EV Charging Station

grid and DERs are only able to supply $50 \mathrm{~kW}$, the demand is shifted to next hour. At 19:00 hours, EV-CS has been provided the rest of the power for it's load.

Figure 2.16 demonstrates the optimized grid schedule to minimize the cost. We can see during high price periods, grid is providing minimum load while depending on all the DERs. 


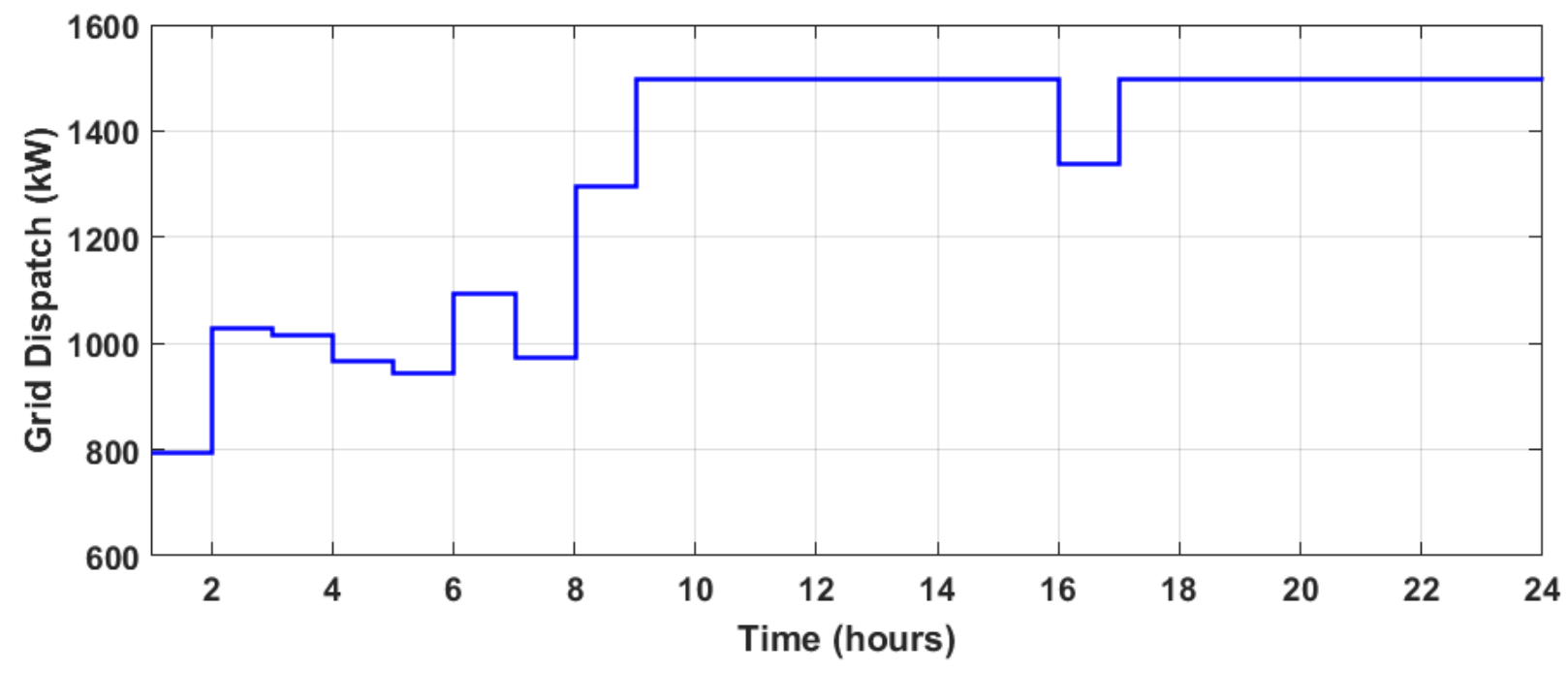

Fig. 2.16 Optimum Grid Dispatch Schedule

\subsubsection{Social Welfare Maximization for EV Charging}

This section presents the simulation results for the proposed primal dual algorithm. In our simulation model, we have considered two cases for two level of penetration of EVs. Each consumer is assumed to have an EV which needs to be charged. The objective is to minimize the cost incurred during charging of vehicles and the power allocation during the peak load for profit of energy provider.

\subsubsection{Convergence of Primal Dual Dynamics}

Figure 2.17 shows the convergence of the primal dual dynamics which justifies the feasibility of the solution to the welfare maximization problem. Primal dual gradient solution is only valid if the optimal values of the solution converges asymptotically. We have considered 100 vehicles for the study. Figure 2.17 shows the convergence for 10 vehicles. We changed the parameters and showed the results for 100 vehicles in figure 2.18. It is evident from both the figures that the optimal values of charging demand converge to its optimal value. This convergence proves that the solution of our objective function is the optimal solution and satisfying the KKT conditions of optimality.

\subsubsection{Energy Management}

In this section, we discuss the energy management using optimal EV charging. We have considered a Time of Use (ToU) pricing for an average summer day to show the impacts of optimal charging. Figure 2.19 presents the charging demand in case of energy management and without energy management (EM) for two vehicles. 


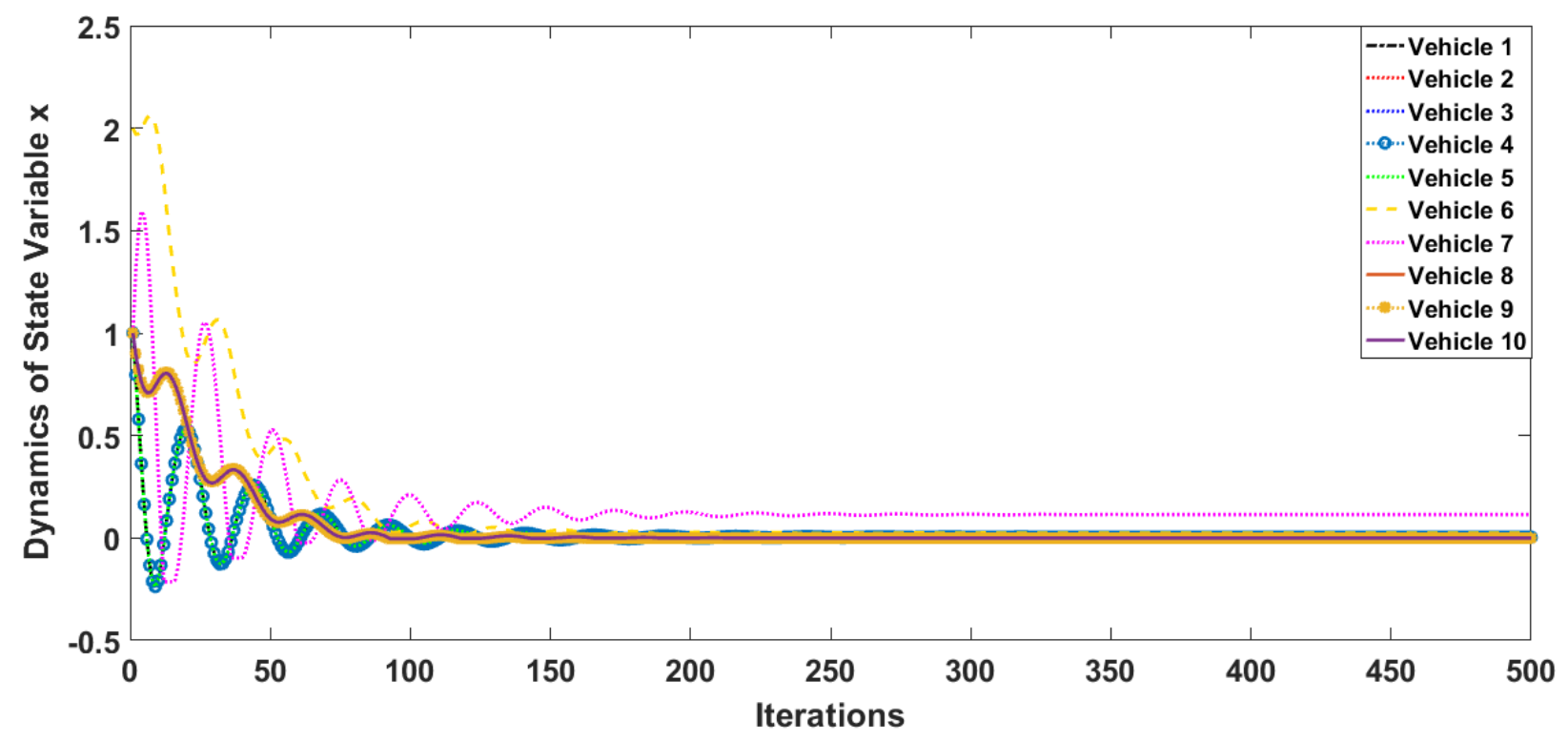

Fig. 2.17 Control Trajectories for $10 \mathrm{EVs}$

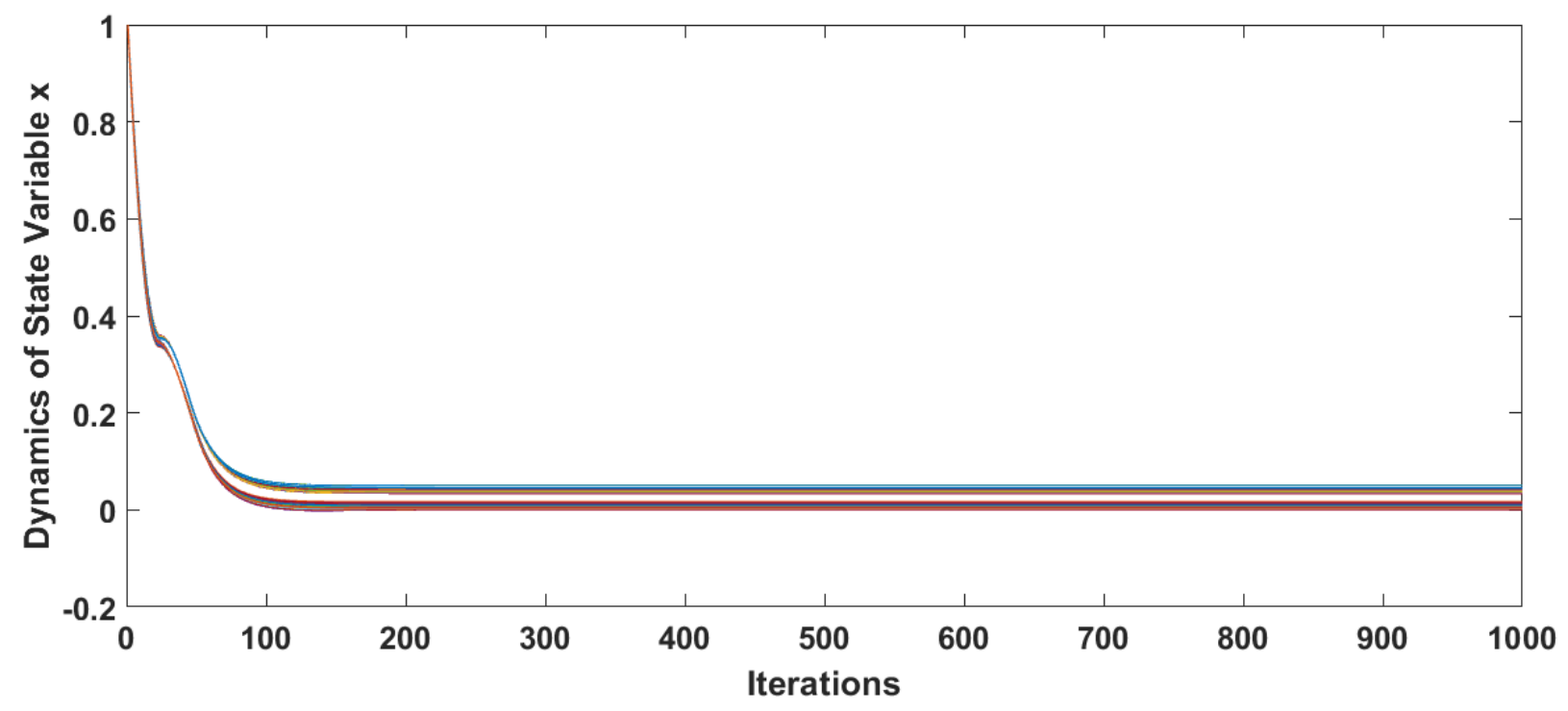

Fig. 2.18 Control Trajectories for $100 \mathrm{EVs}$

Although we have considered 100 vehicles, for the sake of clarity, we have shown results for three vehicles. As shown in figure 2.19, during the peak pricing hours from 11 am to $7 \mathrm{pm}$, with the energy management, the vehicle owner reduce their charging power to due to the high electricity prices. During the non peak hours specifically early morning and late night, the vehicles charging power found to be increasing in EM scenario as seen in the optimized charging schedules of vehicles. 


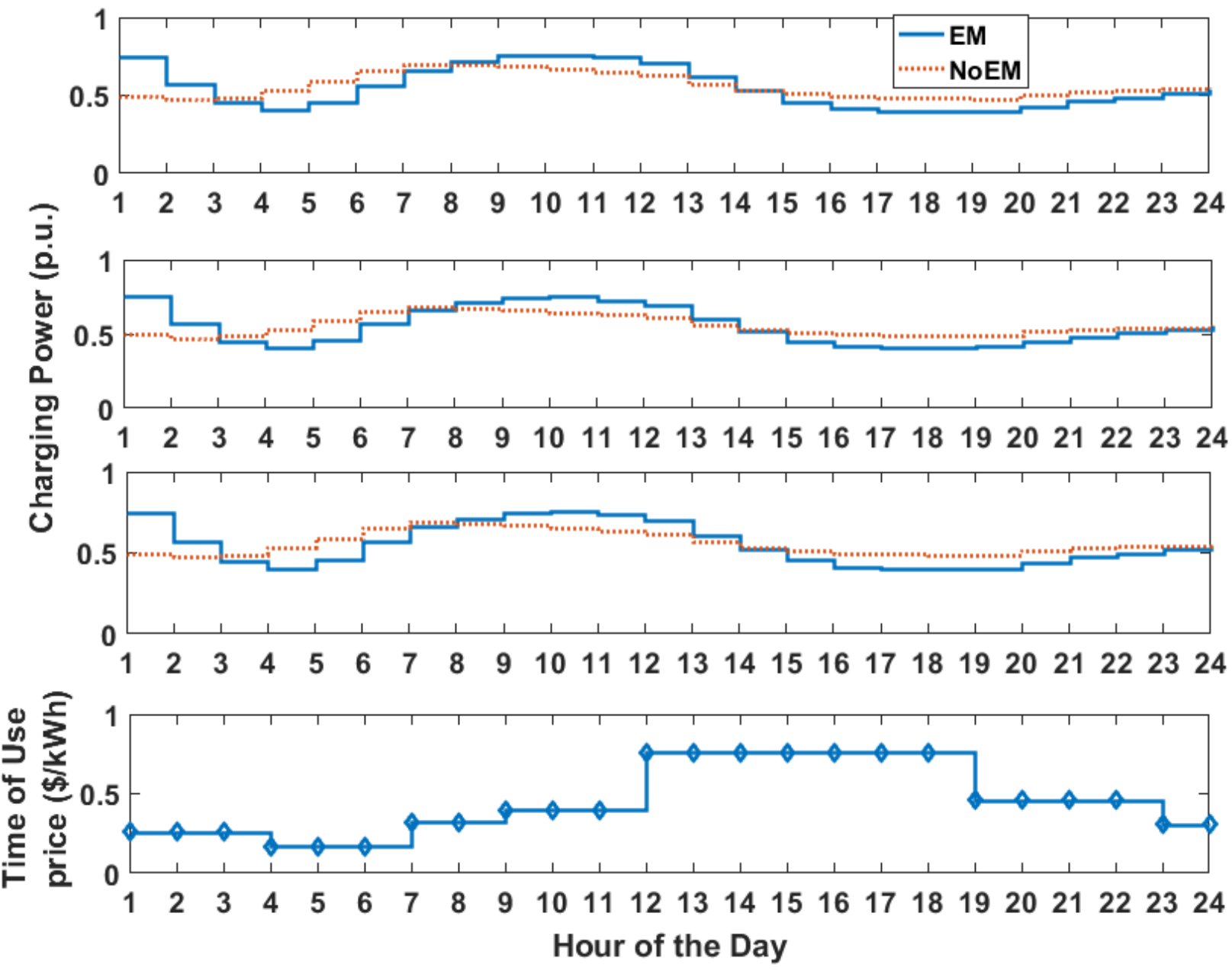

Fig. 2.19 Comparison of Charging Demand with and without Energy Management

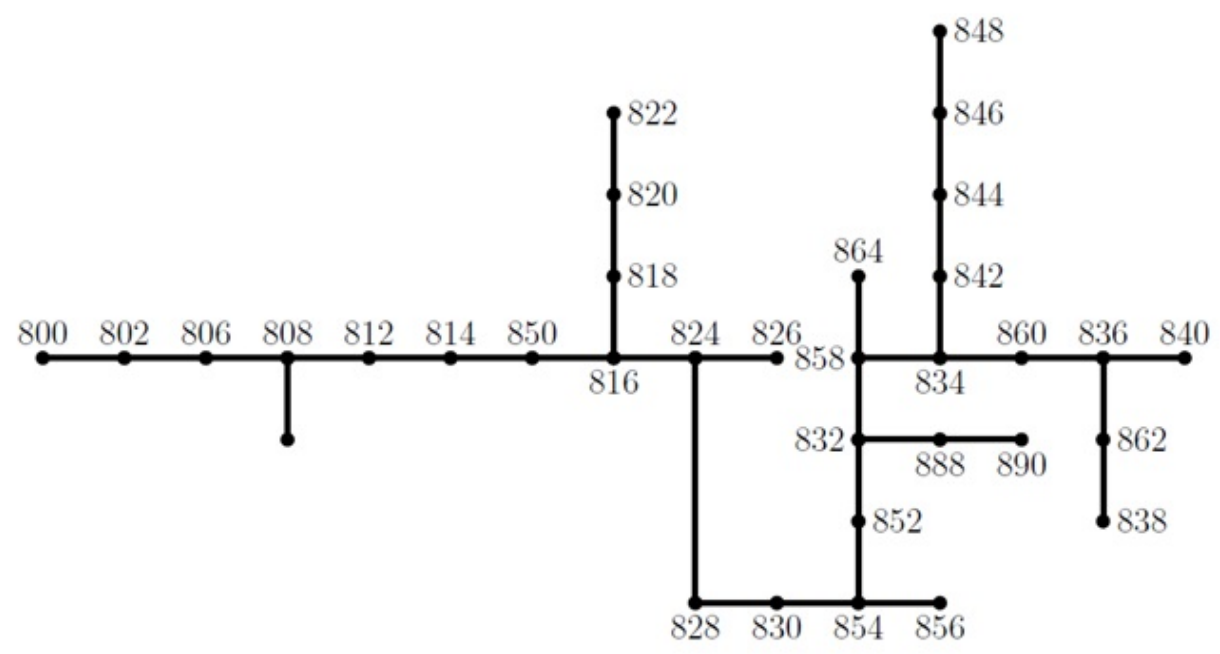

Fig. 2.20 IEEE 34 Node Test Case [53] 
Table 2.5 Load Data

\begin{tabular}{|c|c|c|c|c|c|c|c|}
\hline Node & Ph-1 & Ph-1 & Ph-2 & Ph-2 & Ph-3 & Ph-3 & Load \\
\hline 844 & 135 & 105 & 135 & 105 & 135 & 105 & Y-Z \\
\hline 860 & 20 & 16 & 20 & 16 & 20 & 16 & Y-PQ \\
\hline 890 & 150 & 75 & 150 & 75 & 150 & 75 & D-I \\
\hline
\end{tabular}

In the case of without EM, we have omitted the $\lambda$ dynamics, which indicates the non optimized charging demand. The trade-off parameter between cost and comfort $(\gamma)$ would also be very high. We have assumed the charging efficiency of each EV as 0.92 and battery capacity between 12-15 $\mathrm{kWh}$ for all the EVs.

\subsubsection{Load Flow Analysis}

Load flow analysis plays an important role to calculate the node voltages, branch currents, power losses, and voltage regulation in a power system. Load flow provides the steady state operating conditions. Figure 2.20 shows the schematic diagram of IEEE 34 node test case, used in this analysis. Node 844,860 , and 890 represents the 3 charging stations, charging total 100 EVs.

In this section, we have applied load flow to investigate the effects of energy management of EVs on the node voltages and eventually on the overall system. The load flow is performed after the optimization is run. The power flow constraints were not included in the our optimization problem. The optimal charging profile act as a load (as we are considering only grid to vehicle operation) and was added to the load at the test nodes. The load data at the test nodes is given in table 2.5. Load flow is carried out using OpenDSS MATLAB COM interface. Figure 2.21 illustrates the voltage profiles at the test nodes. The voltages were compared for the two cases 1) with energy management and 2) without energy management. It is observed from the figure that these test node voltages are in the permissible range with the optimized charging load of EVs. This proves that the optimized solution is feasible with the system.

\subsection{Conclusion}

The outcome of the load variance optimization produces proper charging profile of PHEV that has potential to reduce burden on the grid because of the PHEVs integration. This chapter presents an optimized strategy for optimal charging profile of PHEVs for V2G and Grid to vehicle modes. This optimized charging profile for each node helps the grid to control the peak load at times. Considering profits of both utility and EV owners, maximization of social welfare problem was 

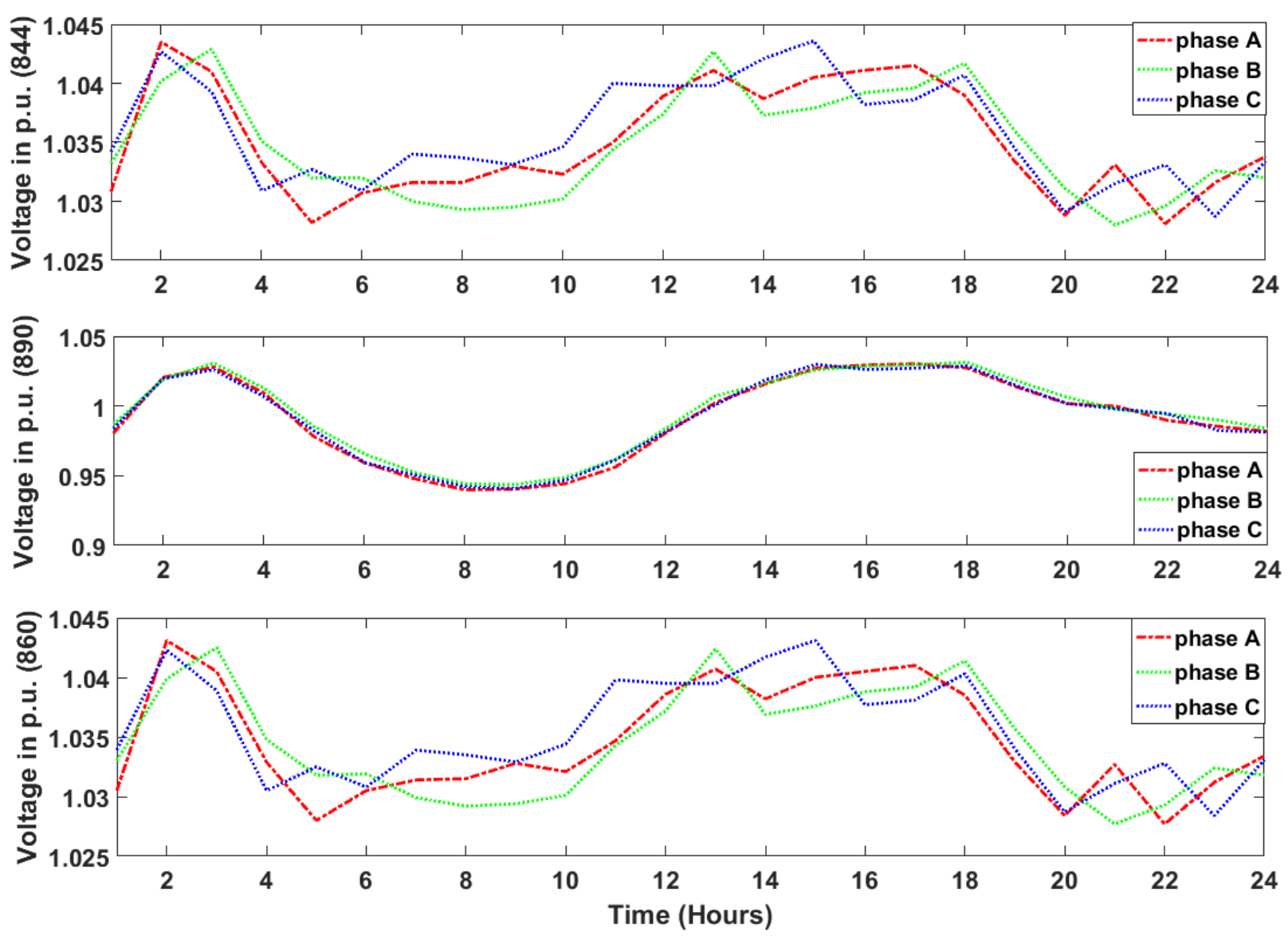

Fig. 2.21 Voltage Profile at the Node 860, 844, and 890

solved using primal dual approach. We have formulated a concave-convex optimization problem with equality constraints.

The optimal scheduling of DERs including the EV charging station, and BESS connected to a community microgrid considering all the operational limits like active power balance equation, depth of discharge and state of charge of BESS were computed as well. The optimal dispatch problem was solved using MATLAB LP solver and considered the operational cost minimization. The feasibility of our results have been verified by performing the load flow analysis on IEEE 13 node and IEEE 34 node test cases. 


\section{CHAPTER 3}

\section{Vehicle-to-Grid Integration for enhancement of Grid: A Distributed Resource Allocation Approach}

\subsection{Introduction}

In the future grids, to reduce greenhouse gas emissions Electric Vehicles (EVs) seems to be an important means of transportation. One of the major disadvantages of the future grid is the demandsupply mismatch which can be mitigated by incorporating the EVs into the grid. This chapter introduces the concept of the Distributed Resource Allocation (DRA) approach for incorporating a large number of Plug-in EV (PEVs) with the power grid utilizing the concept of achieving output consensus. The charging/discharging time of all the participating PEVs are separated with respect to time slots and are considered as strategies. The major aim of this chapter is to obtain a favorable charging strategy for each grid-connected PEVs in such a way that it satisfies both grid objectives in terms of load profile smoothening and minimizing of load shifting as well as economic and social interests of vehicle owners i.e. a fair share of the rate of charging for all connected PEVs. The three-fold contribution of this chapter in smoothening of load profile, load shifting minimization, and fair charging rate is validated using a representative case study. The results confirm improvement in load profile and also highlight a fair deal in the charging rate for each PEV.

\subsection{Literature Survey}

In the future smart grids, electric vehicles (EVs) offer more attractive transportation options in concern with increasing gasoline prices and environmental issues [54][55]. In the case of plug-in hybrid EVs (PHEVs) for powering the electric motor, batteries are used whereas, in the case of an internal combustion engine the power is supplied using different fuel such as diesel or gasoline. The plug-in EVs (PEVs) can be operated in two possible operating modes, one in grid-to-vehicle $(\mathrm{G} 2 \mathrm{~V})$ mode wherein the vehicle uses power from the grid to charge batteries and other in vehicleto-grid (V2G) mode in which the grid receive power by discharging the vehicle's batteries [56]. The introduction of the $\mathrm{V} 2 \mathrm{G}$ concept has captivated curiosity from grid operators as well as PEVs 
owners. However, for realizing the benefits of the V2G concept convenient recharging options and availability of electricity supplies are mandatory. In the V2G concept, the residual energy of the $\mathrm{EV}$ batteries is generally utilized for facilitating the requirement of grids.

The total charging demand of EVs when integrated into the grid constitutes a significant load. The total load on grid increases by an average of $18 \%$ due to EVs charging and this unpredictable load lead to the unreliability of the grid [57]-[58]. In [59], the recharging time of EV and its effects on utilities was studied. The effects of batteries long charging cycle were highlighted by [60]. To avoid peak load time intervals of load cycles a time-shifted fast charge at night time is proposed by [61]. Designing appropriate controllers for stabilization of frequency and modeling of PHEVs with a micro-grid system was studied in [62].

The enhancement of power grid operating conditions such as increasing load factor and reducing power losses by determining the optimal charging profile is claimed by [63]. In [64], an optimization technique based on a genetic algorithm for maximizing the benefits of EVs batteries utilization as an energy storage system in the grid was presented. In a V2G market, the authors of [65] highlighted the modeling of interactions among aggregators and EVs. For charging pattern, an optimization mechanism to concurrently minimize the total degradation of battery health and also the total cost of fuel and electricity over a naturalistic drive cycle of 24 hours was derived by [66].

With the introduction of V2G technology various services with respect to grid like valleyfilling [67], peak shaving [67, 68], compensating reactive power [69, 70], regulation of voltage and frequency[71, 72], and spinning reserve [73] can be achieved. In view of grid peak shaving, the power loss of the distribution network reduces, power quality improves, with a probable increase in the life of transformers. With these improvements, utilities can handle more number of loads without any further extension in the existing network.

In a centralized approach of energy trading [74],[75] a control center or aggregator is responsible for coordinating the discharging of each EVs to meet the demand of network. This approach results in a bidirectional flow of power between the PEVs and the aggregator. However, in contrast to the centralized approach, EVs can decide its own discharging pattern in case of the decentralized approach[14, 76, 77]. The authors in literature[67, 68, 78-80] highlighted the strategies available for peak shaving, wherein [68] and [78] a decentralized approach was adopted for PEVs discharging strategies. However, these approaches fail to guarantee the desired peak shaving, and hence there is a strong need for well-coordinated charging/discharging strategies. The V2G schemes described in $[67,79,80]$ supplies the load demand by discharging PEVs into the grid with proper tracking of the reference line (load demand). The peak shaving achieved in [79] is limited whereas, in the case of $[67,80]$ the satisfactory performance of the algorithm is only possible if there is a high penetration of PEVs. In [81] an algorithm for peak shaving is proposed which provides de- 
sired characteristics even at low penetration rates of PEVs. Similarly, [82] designed an algorithm for the implementation of the $\mathrm{V} 2 \mathrm{G}$ concept with the consideration of reactive power management. Moreover, the authors of [67] and [80] fail to incorporate PEV stochastic nature in terms of mobility leading to inaccurate tracking of the reference line. Furthermore, literature $[67,68,78$, 80] overlooks the requirement of minimum charge required for PEVs to drive back in case of an emergency.

As described in [14] and [83] for a decentralized approach, the complete system is divided into small sub-parts, where each small sub-parts based on the information available from the rest of subparts of the complete system solves an optimization problem. One of the methods which utilize such an approach is the Distributed Resource Allocation (DRA) which uses output consensus. In DRA, for achieving a desirable global state the sub-parts coordinate with each other and make decisions based on the information available locally. The authors in [84] and [85] has explored the DRA approach for PEV integration with the grid, however, the number of PEVs considered is only six.

In this chapter, the problem of finding the optimal charging strategy of the large number of PEVs integrated with a microgrid is considered. PEVs are connected to the microgrid for charging their batteries to the desired capacity. The microgrid supplies power to residential and industrial regions in addition to the charging of PEVs. The concept of DRA is used to calculate the optimal charging strategy of each PEV taking into consideration the load profile smoothening of the grid. A payoff function is formulated for each PEV using smoothening and commitment factors such that reaching of consensus of payoff function of every PEVs gives us the optimal charging strategy in terms of objectives of the grid such as load profile smoothening and prevention of load shifting. The commitment factor is decided by each PEV individually and the smoothening factor is decided by the utility grid.

The major contributions are as follows:

i) DRA approach is applied to obtain a charging strategy that guarantees smoothening of load profile considering all PEVs plugged into the microgrid.

ii) By implementing the error variable in the defined payoff function for the DRA approach gives a fair deal to each PEV with respect to charging rates based on their commitment factors.

The rest of this chapter is organized as follows: Section 3.3 focuses on the problem statement and the associated analogies for the DRA approach. Section 3.4 introduces the prerequisite for understanding the load management problem. Section 3.5 presents the features of the DRA approach considering the output consensus. Section 3.6 highlights the proposed approach application for 
PEV load management. Section 3.7 provides the representative case studies and results to confirm the claim and Section 3.8 concludes with the possible future extension of the work

\subsection{Problem Statement and Analogies}

For supplying power to two different types of customers i.e. fixed and transient as shown in figure 3.1 a distribution type of transformer has been considered. The industrial regions and occupational areas are considered fixed customers and have a load profile which is constant for a long-period of time. In contrast, PEVs are designated as transient customers and have load profile varying with respect to time.

In view of electricity demand variation, for different time instants, different payoff functions are proposed thus there are $k$ time slots for equivalent $k$ different payoff functions and also a PEV charger can be a single-phase or three-phase. Consider an array $A$ where each element represents the active power supplied to the customer by the grid at time slot $k$. The elements of arrays $A$ is given as follows:

$$
A_{k}=a_{k}+\sum_{i=1}^{N} x_{k}^{i} / t_{k}^{i}
$$

where $a_{k}$ represents active power supplied by the distribution transformer at $k^{\text {th }}$ time slot when no PEVs are present. Similarly $x_{k}^{i}$ represents active power of $i^{\text {th }}$ PEV at time slot $k$.

For the energy population, the proposed analogies can be illustrated in terms of strategies [86]. In short, there are $k$ strategies that depend on the values of $k$, where each strategy provides a defined payoff so that individuals can settle on it. As described earlier, fixed customers have strategies constant over a period of time. However, transient customers have the goal of minimizing their cost by changing their strategies from time to time and hence described as transient. In order to satisfy its objectives PEVs if required can discharge their batteries for a certain time period. The discharged energy of the PEV with help of grid can be supplied to the fixed population and in return, the part of the payoff for that particular time slots will be provided to defined PEV. This results in the transient population forcing a fixed population for mitigating to other strategies. Even though the time changes, the whole population covering all strategies remains the same.

As the grid has imposed different payoff functions for different time slots, it is desired to compute the energy consumption of each PEV for different time slots in such a way that all the PEVs act together for providing the grid with several beneficiary services. The beneficiary services range from grid load profile smoothening and load shifting minimization as well as obtaining the

fair share of charging scheme where each PEV is able to get their rate of charging close to their expected rate of charging in the defined time frame. 


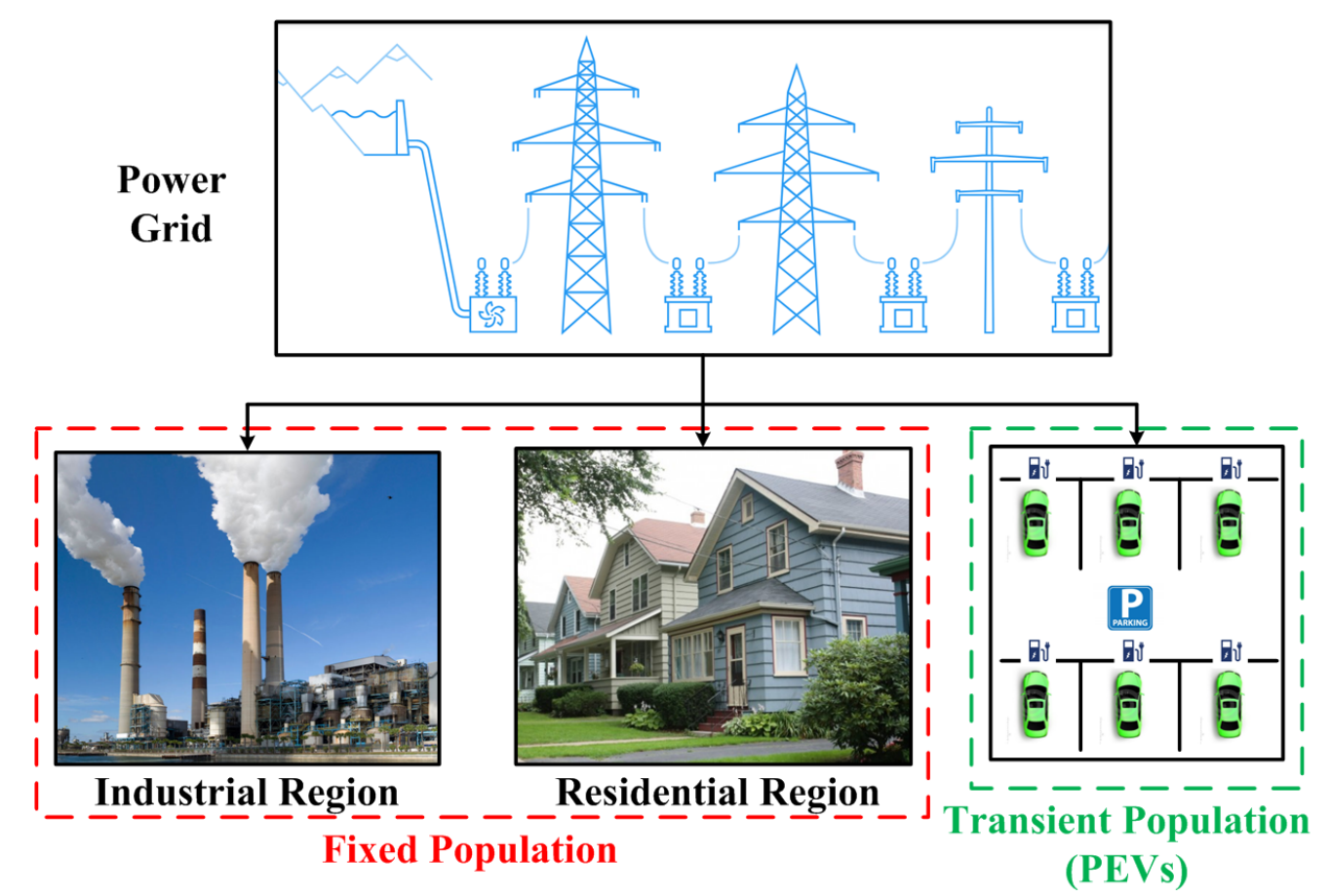

Fig. 3.1 The connection of Power Grid with the transient and fixed population.

\subsection{Preliminaries for DRA approach and barrier function formulation}

\subsubsection{Graph Theory}

The multi-agent system considered here allows the agents to exchange their information using a communication graph which is modelled with the help of a graph. The triplet $\mathcal{C}=(\mathcal{S}, \mathcal{L}, \mathcal{A})$ is used for the mathematical representation of the graph. In the graph, the set of nodes is represented by $\mathcal{S}=\{1, \ldots, K\}$, the set of edges connecting the nodes is represented by $\mathcal{L} \subseteq \mathcal{S} \times \mathcal{S}$ and $\mathcal{A}$ represents a $K \times K$ non-negative matrix. The values of the elements of $\mathcal{A}$ are such that $a_{k j}=1$ for all $(k, j) \in \mathcal{L}$, and $a_{k j}=0$ for all $(k, j) \notin \mathcal{L}$. The agents and communication channels of the multi-agent system are represented by the nodes and edges of the graph respectively. Hence, agents $k$ and $j$ are connected and can communicate with each other if and only if $(k, j) \in \mathcal{L}$. The neighbours of node $k$ i.e. all the nodes that can communicate and share information with node $k$ are represented by $\mathcal{N}_{k}=\{j \in \mathcal{S}:(k, j) \in \mathcal{L}\}$.

The following assumptions are considered for the graphical modeling of the multi-agent system:

i) $a_{k k}=0 \quad \forall k \in \mathcal{S}$ i.e. no self-loops are present.

ii) $a_{k j}=a_{j k}$ i.e. communication channels are bidierctional.

The matrix $L(\mathcal{C})=\left[l_{k j}\right]$ is the $K \times K$ graph Laplacian matrix of $\mathcal{C}$ and can be defined as follows: 


$$
l_{k j}=\left\{\begin{array}{l}
\sum_{j \in \mathcal{S}} a_{k j}, \quad \text { if } \quad k=j \\
-a_{k j}, \quad \text { if } \quad k \neq j
\end{array}\right.
$$

\subsubsection{System Passivity Structure}

The convergence of the DRA algorithm using consensus protocol can be done utilizing the concept of the passivity framework. The concept of passivity theorem as described in [87] is given as follows.

A dynamical system can be represented by the state model as follows:

$$
\begin{aligned}
\dot{x} & =f(x, u) \\
y & =h(x, u)
\end{aligned}
$$

where $f: R^{n} \times R^{p} \rightarrow R^{n}$ is locally Lipschitz, $h: R^{n} \times R^{p} \rightarrow R^{p}$ is continuous, $f(0,0)=0$, and $h(0,0)=0$. The total number of inputs of the system is equal to the total number of outputs. The system represented by (3.3) would be passive if there exists a continuously differentiable positive semidefinite function $V(x)$ (called storage function) such that

$$
u^{T} y \geq \dot{V}=\frac{\partial V}{\partial x} f(x, u), \quad \forall(x, u) \in R^{n} \times R^{p}
$$

Moreover, it is said to be

- lossless if $u^{T} y=\dot{V} \quad \forall(x, u) \in R^{n} \times R^{p}$.

- strictly passive if $u^{T} y \geq \dot{V}+\psi(x)$ for some positive definite function $\psi$, and for $\forall(x, u) \in$ $R^{n} \times R^{p}$.

\subsubsection{Barrier Function Framework}

In many practical applications, limited resource availability, system design limitations, etc. impose various restrictions on the state of system. Hence, for the proper operation, it is crucial that the dynamics evolution (3.3) should be bounded to predefined feasible region of the state space. Consider constraints in the form of upper and lower bound on the state value $x$ given as $(l, m)$. To incorporate this constraint, this chapter utilizes barrier formulation $\beta(x)$ provided in [88].

$$
\beta(x)=\frac{1}{l-x}+\frac{1}{m-x}
$$

The barrier function $\beta(x)$ has the following properties: 
- $\beta(x)$ is monotonically increasing continuous function defined in $(l, m)$.

- $\beta(x) \rightarrow-\infty$, when $x \rightarrow l$.

- $\beta(x) \rightarrow \infty$, when $x \rightarrow m$.

Barrier function is considered as the derivative of a convex function which obstructs the control signal from violating its feasible domain.

\subsection{Features of DRA}

A multi-agent system of $n$ agents is considered which is connected by a communication network. The weighted graph $\mathcal{C}=(\mathcal{S}, \mathcal{L}, \mathcal{A})$ is used to characterize this system. The dynamical model of the system is represented by the following differential equations:

$$
\Gamma_{k}^{S}:\left\{\begin{array}{l}
\dot{x}_{k}=f(x) \\
y_{k}=g(x)
\end{array}\right.
$$

where the system as a whole is represented as $\Gamma_{k}^{S}$, the output of subsystem $k$ is represented by $y_{k} \in R$ and the state of subsystem $k$ is represented by $x_{k}$. Driving the system to a desired global state where grid objectives are met is the main objective of all agents as mentioned in Section 3.3 and Section 3.4. However, each agent has only partial information of the system. A situation is considered where the agent only knows the information of its output and the output of its neighbours i.e., the value of $y_{k}$ and that of $y_{j}$ for all $j \in \mathcal{N}_{k}$ is known by the $k^{t h}$ agent. The control law of each agent is formulated by utilizing all the available information which drives their $\dot{x}$. This is shown in the following equation:

$$
\Gamma_{k}^{C}: \dot{x}_{k}=u_{k}\left(y_{k}, y_{j}\right), \forall j \in \mathcal{N}_{k}
$$

\subsubsection{Control Objective}

In many applications, the desired global state in a multi-agent system can be achieved by reaching consensus, i.e., all subsystems reaching the same output. Such problems are called output consensus problems. The definition of output consensus as defined in [88] is:

Definition 3.5.1. Consider the set of subsystems given in (3.6) and (3.7). It can be said that output consensus is reached if $\lim _{t \rightarrow \infty}\left|y_{k}(t)-y_{j}(t)\right|=0$, for all $i, j=1, \ldots, n$ where $y_{k}(t)$ is the output of the subsystem $i$ at time $t$. 
In this chapter, output consensus is achieved utilizing the constraint applied to the state variables:

$$
\sum_{k=1}^{n} x_{k}^{i}=X
$$

where the sum of all the state values in the given time frame is represented by $X \in R$. The electrical energy transferred between the PEVs and the grid is represented by $x_{k}^{i}$ and the total amount of electrical energy required to completely charge the battery of each PEV is represented by $X$. The control objective of the multi-agent system can be summarized as follows:

i) Satisfying the constraint (3.8).

ii) Driving (3.6) to output consensus.

\subsubsection{Dynamics of Resource Allocation}

The desired global state is achieved by designing local control laws $u_{1}, u_{2}, \ldots, u_{n}$ to be applied to the multi-agent system. The proposed DRA dynamic equation is:

$$
u_{k}\left(y_{k}, y_{j}\right)=\sum_{j \in \mathcal{N}_{k}} a_{k j}\left(y_{j}-y_{k}\right), \forall k=1, \ldots, K \text { and } \forall j \in \mathcal{N}_{k}
$$

The constraint (3.8) easily satisfies (3.9) if following conditions are met:

i) $\sum_{k=1}^{K} x_{k}^{i}(0)=X$

ii) $\sum_{k=1}^{K} \dot{x}_{k}^{i}=0$

\subsubsection{Convergence to Output Consensus}

The multi-agent system represented by (3.6) utilizing the control law (3.7) can be thought of as a feedback interconnection outlook as displayed in figure 3.2. The proposed DRA dynamical equation (3.9) and its equilibrium point $\left(x^{*}\right)$ of the feedback interconnection must satisfy the Statement 1 which is adapted from [88]:

Statement 1: Consider the feedback interconnection shown in figure 3.2 having its equilibrium point at $x^{*}$ and let the steady state output of $\Gamma^{S}$ be $y^{*}=g\left(x^{*}\right)$. If $u\left(y_{k}, y_{j}\right) \quad \forall j \in \mathcal{N}_{k}$ is given by (3.9) and the communication graph $\mathcal{C}$ is connected, then $y_{k}^{*}=y_{j}^{*} \quad \forall k, j=1, \ldots, K$ where $y_{k}^{*}$ is the $k^{\text {th }}$ element of the vector $y^{*}$. Using the definition of output consensus problem, Statement 1 states that if the equilibrium point $x^{*}$ is asymptotically stable then the output consensus will be obtained. To check the stability of $x^{*}$ the dynamics of $\Gamma^{S}$ and $\Gamma^{C}$ are expressed in error coordinates. 


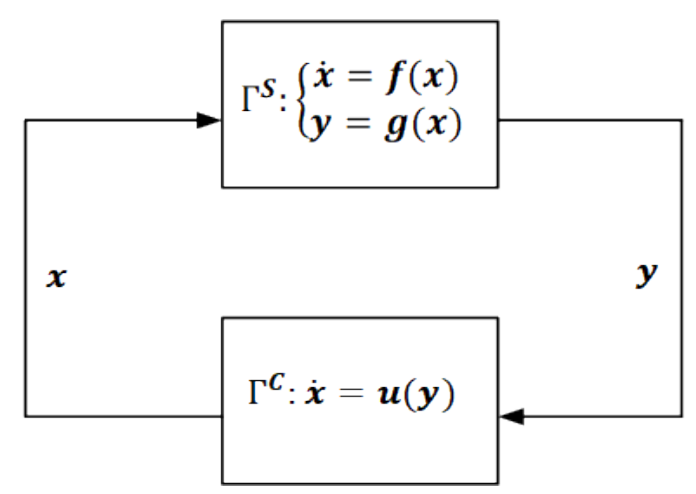

Fig. 3.2 Representation of (3.6) and (3.7) via feedback interconnection outlook.

The system $\Gamma^{S}$ is written in error coordinates as,

$$
\Gamma_{e}^{S}:\left\{\begin{array}{l}
\dot{e}_{x}=f^{e}\left(e_{x}\right) \\
e_{y}=g^{e}\left(e_{x}\right)
\end{array}\right.
$$

where $e_{x}=x-x^{*}$ and $e_{y}=y-y^{*}$. Also $f^{e}\left(e_{x}\right)=f(x)$ and $g^{e}\left(e_{x}\right)=g(x)-g\left(x^{*}\right)$ for all $x \in R^{n}$. Since $x^{*}$ is an equilibrium point of (3.6), it can be seen that $f^{e}(0)=0$, and $g^{e}(0)=0$. Assumption 1 is made on (3.10):

Assumption 1: Consider the dynamical system (3.10). If $f^{e}(0)=0$, then $e_{x}=0$.

The Assumption 1 guarantees the existence of unique rest points for (3.6). Similarly, the dynamics of (3.7) that implements (3.9) is also expressed in error coordinates using Laplacian of $\mathcal{C}$ as:

$$
\dot{e}_{x}=-L(\mathcal{C}) e_{y}
$$

Now consider the Statement 2 given in [88] reformulated as:

Statement 2: The multi-agent system expressed in error coordinates given by (3.9) is passive and lossless from the input $e_{y}$ to the output $-e_{x}$, if $x(0)$ and $x^{*}$ satisfies the resource constraint (3.8), i.e., $\sum_{k=1}^{K} x_{k}^{*}=X$ and $\mathcal{C}$ is connected. The concept of passivity can be explored along with Statement 2 to validate the stability of equilibrium points of (3.6) as described in [88]. Feedback interconnection of two passive systems generally results in stable rest points. This property of passivity is utilized to ensure output consensus is achieved under the configuration as shown in figure 3.2. The Theorem 2 adapted from [88] is used to summarize the requirements to reach output consensus.

Theorem 2: Consider the feedback interconnection of system (3.6) and (3.7) having its equilibrium point at $x^{*}$ where $(8)$ defines the $u(y)$. Following conditions are assumed:

i) The connectivity of the communication graph $\mathcal{C}$ of the system given by (3.7) is assured. 
ii) The resource constraint (3.8) is satisfied by $x^{*}$ and $x(0)$.

iii) Assumption 1 is satisfied by the system (3.6) expressed in error coordinates with respect to $x^{*}$. Moreover it is strictly passive from the input $e_{x}$ to the output $e_{y}$ with radially unbounded storage function.

Then (3.6) reaches output consensus.

\subsection{Application of DRA for load management of PEV}

Based on the proposed DRA dynamical equation (3.6)- (3.7) and properties of output consensus problem, the application of PEVs inclusion with a microgrid is presented.

\subsubsection{Constraints on PEV variables}

Following energy constraints are considered in the application of PEV incorporation similar to the constraints given in [86]

$$
\begin{gathered}
\sum_{k=1}^{K^{i}} x_{k}^{i}=s o c_{K}^{i}-s o c_{0}^{i} \\
x_{k}^{i} \leq \overline{s o c}^{i}-\left(s o c_{0}^{i}+\sum_{\omega=1}^{\Omega} x_{\omega}^{i}-x_{k}^{i}\right), \\
\forall \Omega=\left\{1,2, \ldots, K^{i}\right\}, \forall k=\{1,2, \ldots, \Omega\}, \\
\forall \Omega=\left\{1,2, \ldots, K^{i}\right\}, \forall k=\{1,2, \ldots, \Omega\}, \\
x_{k}^{i} \geq \underline{s o c}^{i}-\left(s o c_{0}^{i}+\sum_{\omega=1}^{\Omega} x_{\omega}^{i}-x_{k}^{i}\right), \\
-t_{k}^{i} \bar{p}^{i} \leq x_{k}^{i} \leq t_{k}^{i} \bar{p}^{i}, \quad \forall k=\left\{1,2, \ldots, K^{i}\right\}
\end{gathered}
$$

where $s o c_{0}^{i}$ represents initial state of charge (in Watt-hour), $s o c_{K}^{i}$ is represented as desired state of charge (in Watt-hour) at the end of time window, $t_{k}^{i}$ is the length of $k^{\text {th }}$ time step (in hours), and $\bar{p}^{i}$ is the nominal power of the charger. Constraint (3.12) is equivalent to the state variable constraint define by (3.8). Constraints (3.13) and (3.14) defines the accumulated state of charge at a particular time instant which is restricted to cross upper limit $\overline{S O C}^{i}$ as well as lower limit $\underline{s O C}^{i}$. Constraint (3.15) defines limits of rate of energy consumption and injection by PEV which in turn depends upon the limits of charger as well as length of time steps. 
The following reactive power constraints are used similar to active power constraints defined precedingly:

$$
\begin{gathered}
\sum_{k=1}^{K^{i}} y_{k}^{i}+s^{i}=0 \\
-\bar{q}_{k}^{i} \leq y_{k}^{i} \leq \bar{q}_{k}^{i}, \quad \forall k=\left\{1,2, \ldots, K^{i}\right\} \\
-Q^{i} \leq s^{i} \leq Q^{i}
\end{gathered}
$$

where $y_{k}^{i}$ defines total reactive power of charger at each time step, $s^{i}$ defines the auxillary slack variables and available reactive power $\bar{q}_{k}^{i}$ is defined as

$$
\bar{q}_{k}^{i}= \pm \sqrt{\left(\bar{p}^{i}\right)^{2}-\left(x_{k}^{i} / t_{k}^{i}\right)^{2}}
$$

The total reactive power $Q^{i}$ available in the time window is defined as

$$
Q^{i}=\sum_{k=1}^{K^{i}} \sqrt{\left(\bar{p}^{i}\right)^{2}-\left(x_{k}^{i} / t_{k}^{i}\right)^{2}}
$$

Equations (3.16) and (3.18) are defined in such a way that sum of all the absolute values of contributions of reactive power of charger in the time window has to be less or equal to $Q^{i}$.

$$
-Q^{i} \leq \sum_{k=1}^{K^{i}} y_{k}^{i} \leq Q^{i}
$$

and the portion of $Q^{i}$ that is redundant in the time window are assigned to the slack variables.

\subsubsection{Output Functions Formulation}

The payoff function of each time step would be the output function of this particular resource allocation problem. Payoff functions are defined in such a way that the objectives of both PEVs owner and grid are met simultaneously. In view of this, a commitment factor $\mu^{i}$ and a smoothing factor $\eta$ are introduced. The commitment factor $\mu^{i}$ is controllable by the owners of PEV and gives a level of choice to them i.e. time duration in which PEV battery should be fully charged. The sudden variation in the profile of active power while transitioning from one time step to next is monitored by $\eta$, the parameter which is controlled by a power grid manager. These factors are defined as

$$
\underline{\mu} \leq \mu^{i}<1, \quad 0 \leq \eta \leq 1
$$




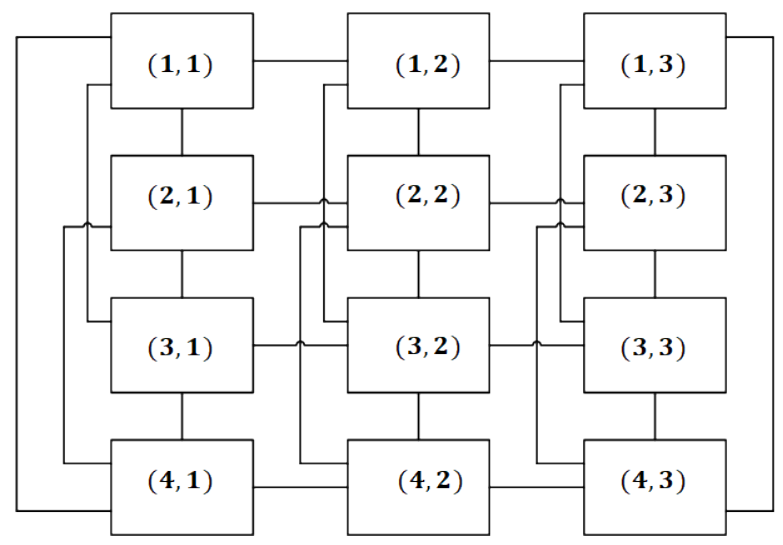

Fig. 3.3 Partial information connectivity diagram for a system of three PEVs connected to the grid with grid providing payoff functions for four time slots.

where $0<\underline{\mu}<1$ is defined as the minimum allowable limit of commitment. For strategies corresponding to active power the payoff functions are defined as:

$$
f_{k}^{i}\left(x_{k}^{i}\right)=-(1-\mu)\left(x_{k}^{i}-x_{k}^{i *}\right) / t_{k}^{i}-\mu \eta A_{k}-\mu(1-\eta)\left(2 A_{k}-A_{k-1}-A_{k+1}\right)
$$

where $x_{k}^{i *}$ is the desired charging rate of the PEV owner's at the $k^{\text {th }}$ time step and the $\mu$ is the mean value of all $\mu^{i}$.

The total output profiles, as well as the profile of active power, are affected by variation in the value of $\mu$ and $\eta$. When $\mu=0$, then smoothening/flattening objectives are neglected, while payoff functions give importance to local references of load distribution. When $\eta=0$ and $\mu>0$, then importance is given to smoothening objective while flattening objectives are ignored. Lastly, when $\eta=1$ and $\mu>0$ then importance is given to flattening objectives while the smoothening objective is neglected. Thus it can be seen from the above cases that there is direct or indirect control of these parameters by a utility grid manager. These parameters are used as an agreement between grid objectives and also social and economic benefits to owners of PEV. For active power strategies the constraints (3.12)-(3.15) are incorporated in the output function by adding the barrier function developed in Section 3.4.3 to the payoff function described in (3.23) Thus, for active power strategies the modified output function is given as:

$$
l_{k}^{i}\left(x_{k}^{i}\right)=f_{k}^{i}\left(x_{k}^{i}\right)+\beta\left(x_{k}^{i}\right)
$$

For active power strategy:

$$
x_{k}^{i} \in(a, b)
$$

where 


$$
\begin{gathered}
a \in \max \left[\underline{s o c}^{i}-\left(\operatorname{soc}_{0}^{i}+\sum_{\omega=1}^{\Omega} x_{\omega}^{i}-x_{k}^{i}\right),-t_{k}^{i} \bar{p}^{i}\right] \\
b \in \min \left[\overline{\operatorname{soc}}^{i}-\left(\operatorname{soc}_{0}^{i}+\sum_{\omega=1}^{\Omega} x_{\omega}^{i}-x_{k}^{i}\right), t_{k}^{i} \bar{p}^{i}\right]
\end{gathered}
$$

The working of the barrier function to satisfy the constraints given in (3.12)-(3.15) for active power strategies can be understood from the example:

Assume the active power strategy $x_{k}^{i}$ to be very close to the upper bound. As a result, the corresponding payoff function value will be higher than that of other function values. This is because $\beta\left(x_{k}^{i}\right)$ is present in the payoff function, given by (3.5), and is a monotonically increasing function that tends to $+\infty$ when $x_{k}^{i}$ gets closer to the upper bound. $\dot{x}_{k}^{i}$ becomes negative (according to (3.7) and (3.9) when the above condition occurs and therefore the value of $x_{k}^{i}$ will decrease and upper bound is not violated. As a result, it can be ensured that the proposed consensus algorithm does not generate a charging rate which is not feasible by the PEV batteries which would make the DRA approach using Consensus protocol not applicable to the present scenario.

\subsubsection{Information Connectivity Graph of Proposed Problem}

There are $K$ strategies in the PEV power grid incorporation problem. For achieving output consensus as described in Section 3.4.1, the connectivity of the strategies is necessary. The equations (3.1), and (3.23), governs the connectivity graph of the proposed strategies. An example of the connectivity graph is shown in figure 3.3 for three PEVs connected to the grid, i.e., $i=\{1,2,3\}$ and grid providing payoff functions for four time slots i.e., $k=\{1,2,3,4\}$ where $(k, i)$ represents the strategy with $k^{t h}$ time slot for $i^{t h} \mathrm{PEV}$.

The figure 3.4 represents the implementation steps. The procedure initiates by determining the total number of PEVs involved in load sharing. Parking lot equipped with such charging functionalities can be one such scenario. Each participating PEV declares its SOC need along with its tendency do aid load sharing in terms of parameter values $\mu$ and $\eta$. The owner can decide these parameters based on various factors such as urgency, total parking period, required SOC, etc. Once all the parameters are finalized, the output function is calculated for each PEV over every time slot. Using this output function value each PEV then evaluates its charging and discharging strategies using the DRA algorithm. This process is repeated until the output consensus is reached. 


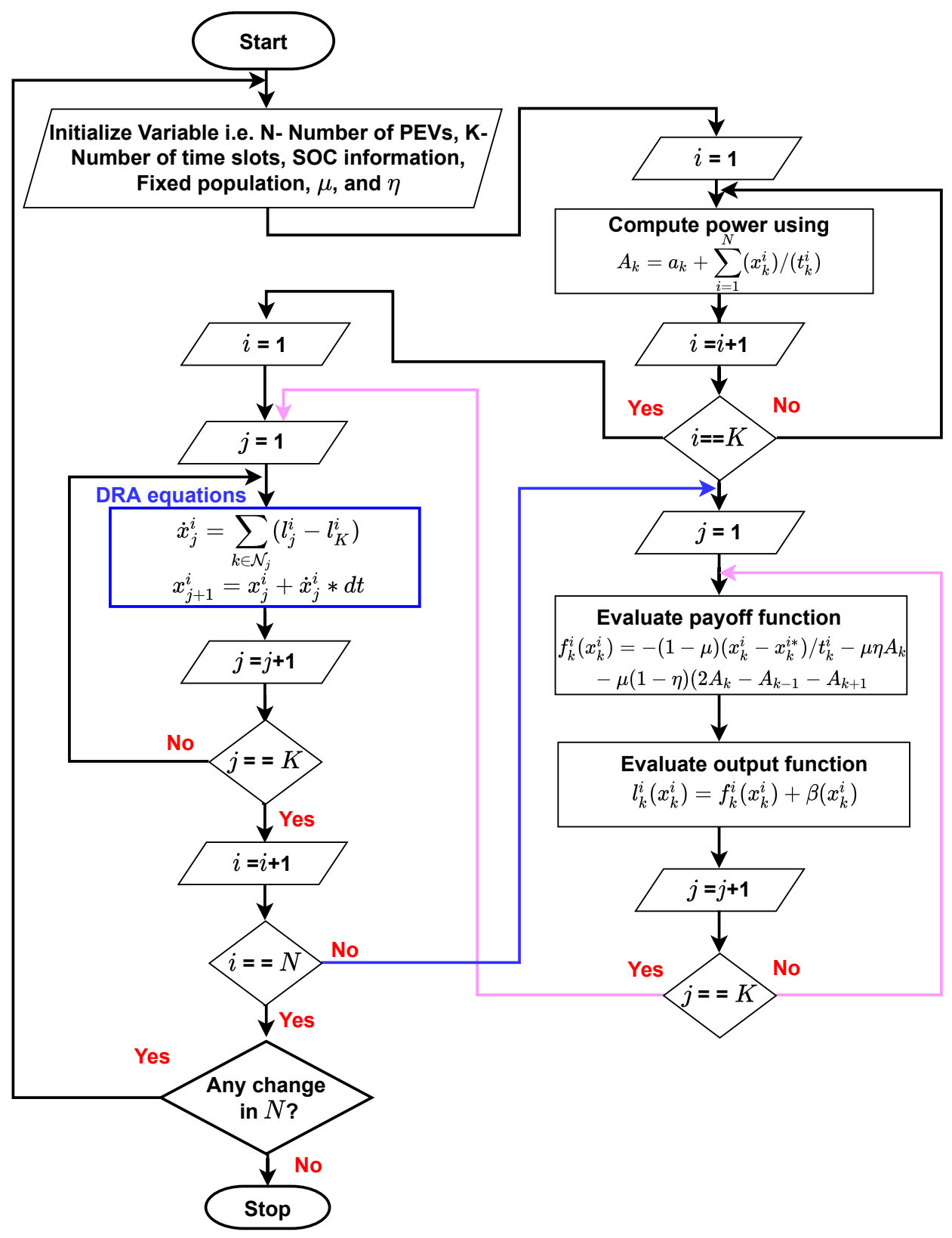

Fig. 3.4 Flowchart for implementation of DRA approach in grid profile enhancement using PEVs

\subsection{Representative Case study and Results for grid enhancement using PEVs}

The representative case study comprises of virtual simulation for a fleet of 40 PEVs over the span of 24 hours. Every PEV is considered to arrive and depart from the charging station according to its own preference. Figure 3.5 represents the cumulative load profile and the respective feature is represented using the black horizontal segments in the start and at the end of every trajectory in figure 3.6 which denotes the absence of the respective PEV due to late arrival and early departure, respectively. Moreover, each PEV is assumed to have different charging requirements. 


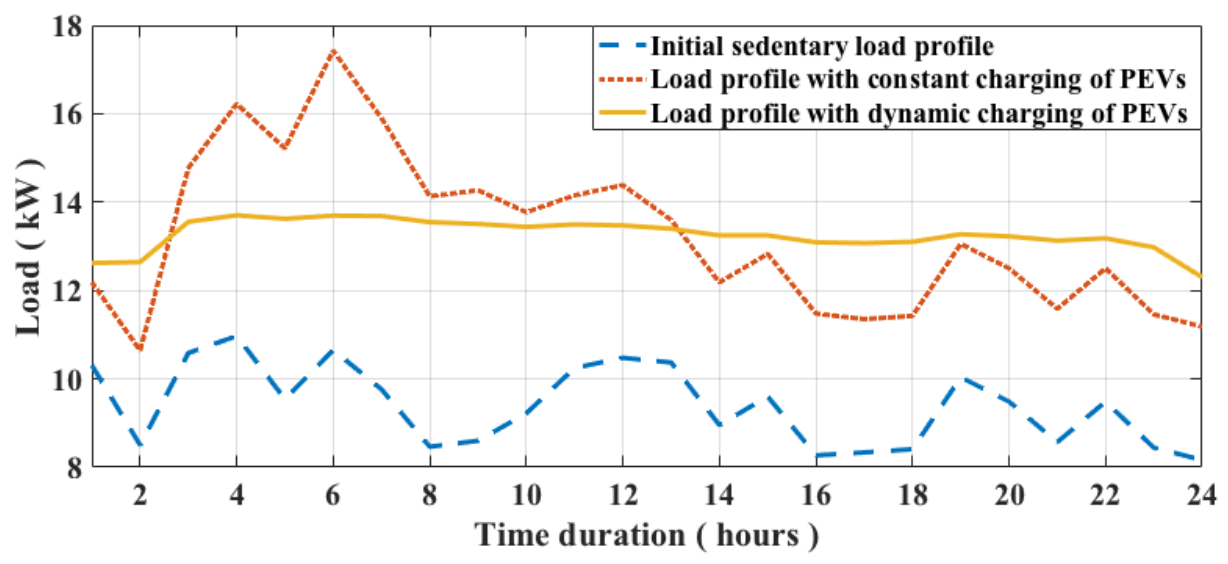

Fig. 3.5 Cumulative load profiles

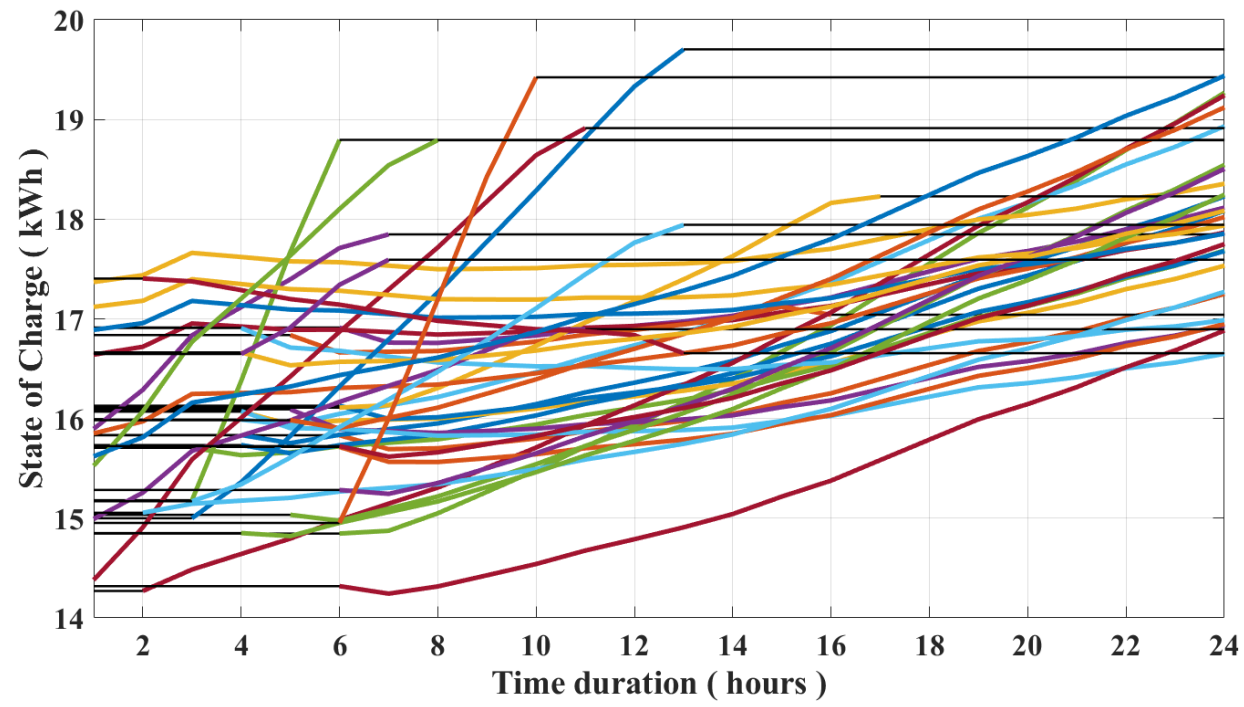

Fig. 3.6 PEV charging and discharging profiles

The minimum allowable state of charge $S O C_{\text {downlimit }}$ for every vehicle is bounded within the band $14 \mathrm{kWh}$ to $16 \mathrm{kWh}$ while the maximum limit of the batteries $S O C_{\text {uplimit }}$ is restricted between $18 \mathrm{kWh}$ and $20 \mathrm{kWh}$. The values of the initial SOC $S O C_{0}$ and desired SOC $S O C_{K}$ for each PEV is bounded by its respective $S O C_{\text {downlimit }}$ and $S O C_{\text {uplimit }}$. Wherein, the value of $S O C_{K}$ is greater than the respective PEV's $S O C_{0}$. Moreover, the charger power limit at any given instance is considered to be $3 \mathrm{~kW}$. In figure 3.5, a comparison is provided between the DRA approach and constant rate approach wherein the PEVs are continuously charged at a constant rate. The load profile in yellow specifies the outcome of the DRA implementation whereas the load profile in red represents the outcome of the application of a constant rate approach. 


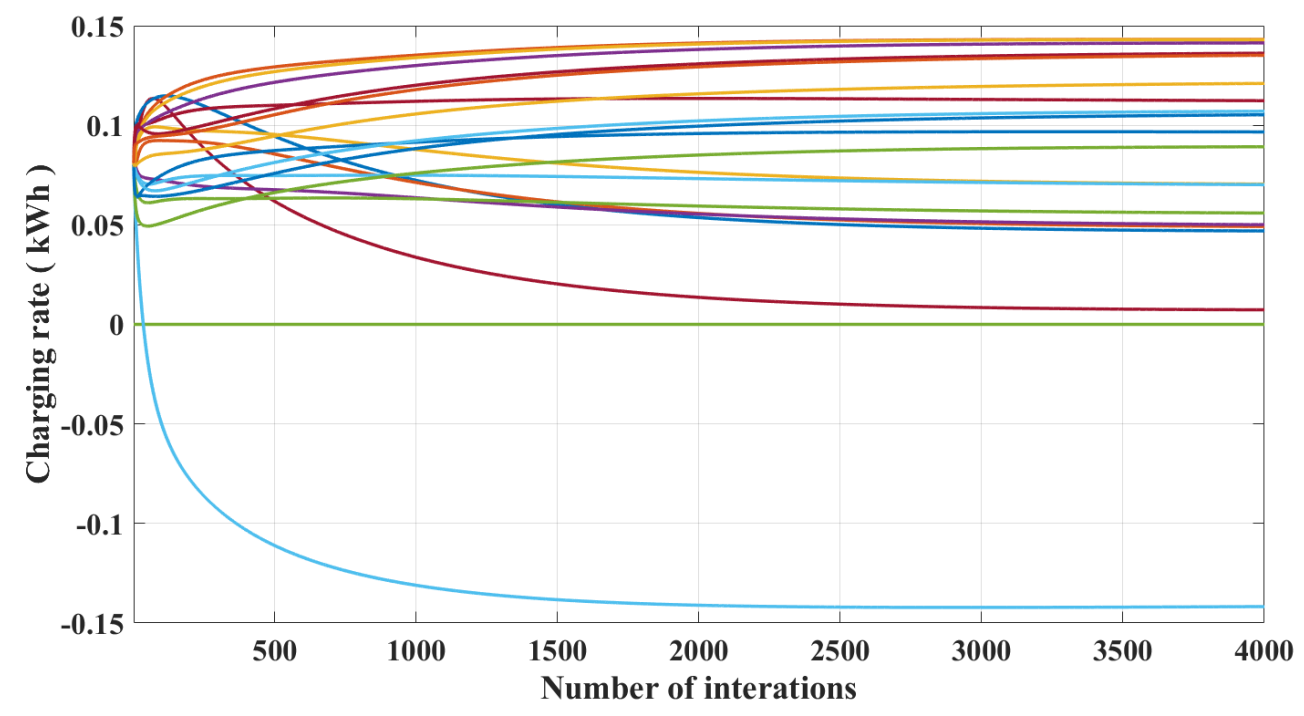

Fig. 3.7 Charging strategies for different PEVs

The overall sedentary population in the absence of PEVs over the duration of 24 hours is represented by the blue color graph represents. In the approach with the constant charging rate, the discharging capability of batteries is not utilized. Compared to the respective implementation result yellow the graph depicting the result of the DRA approach is smooth and flat. This approach not only charges but also discharges the PEV batteries to provide ancillary support to the grid. In figure 3.6, it can be seen that not every trajectory is monotonous, the random troughs in the individual trajectory represent the process of battery discharging to share the high demand of the sedentary population, similarly, crest region represents the charging process of PEVs when the cumulative load on the grid is below average. The convergence of this approach is represented in figure 3.7. In this, each line corresponds to the particular time slot, i.e. the total number of trajectories in figure 3.7 is equal to 24 .

\subsubsection{Importance of design parameters $\mu$ and $\eta$}

The traditional way of charging with approximately constant charging rate results into the additive load at each time step as shown in figure 3.5 red graph. This approach is referred to as customercentric implementation because in this no adjustments in the charging process are introduced to have smooth load variations over time. On the other hand, with the DRA methodology, it is possible to adjust charging behavior to have trade-off among the customer-centric implementation and grid assisting implementation. The careful selection of design parameter $\mu$ and $\eta$ within the constraints (3.22) can provide different grid assessing implementation approaches while satisfying end-user requirements. Figure 3.8 shows one such implementation scenario. 


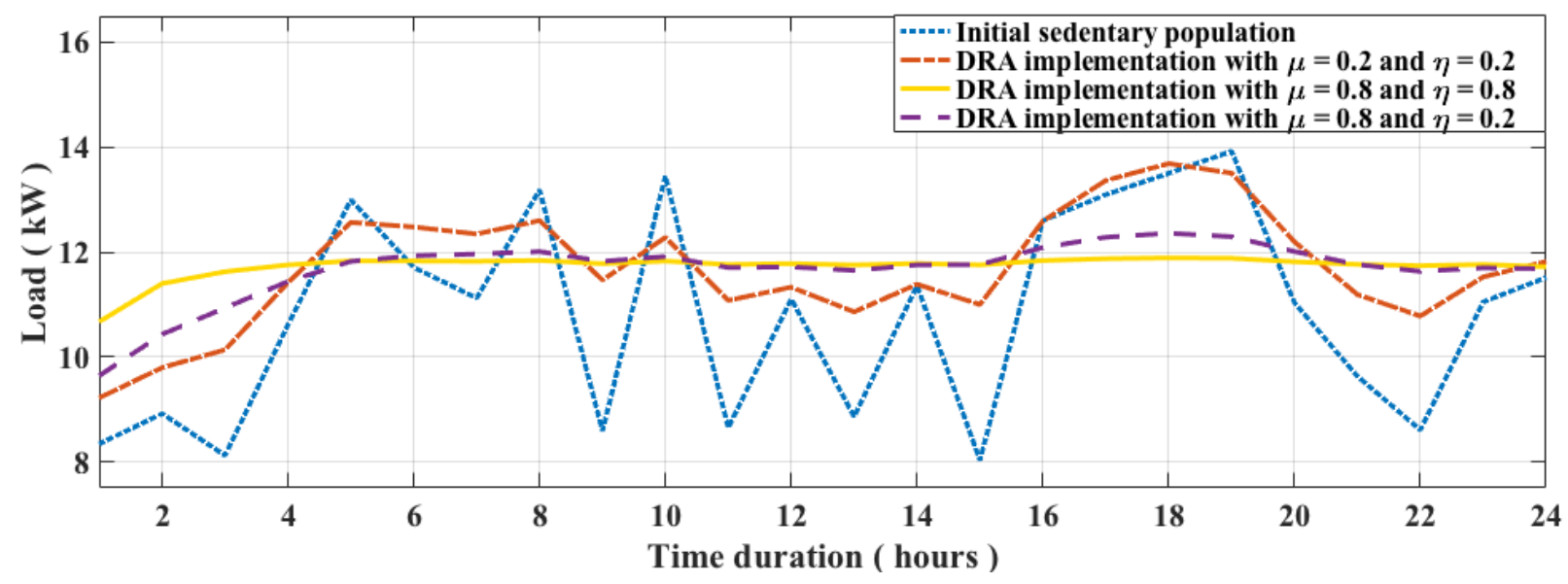

Fig. 3.8 Variation in design parameters

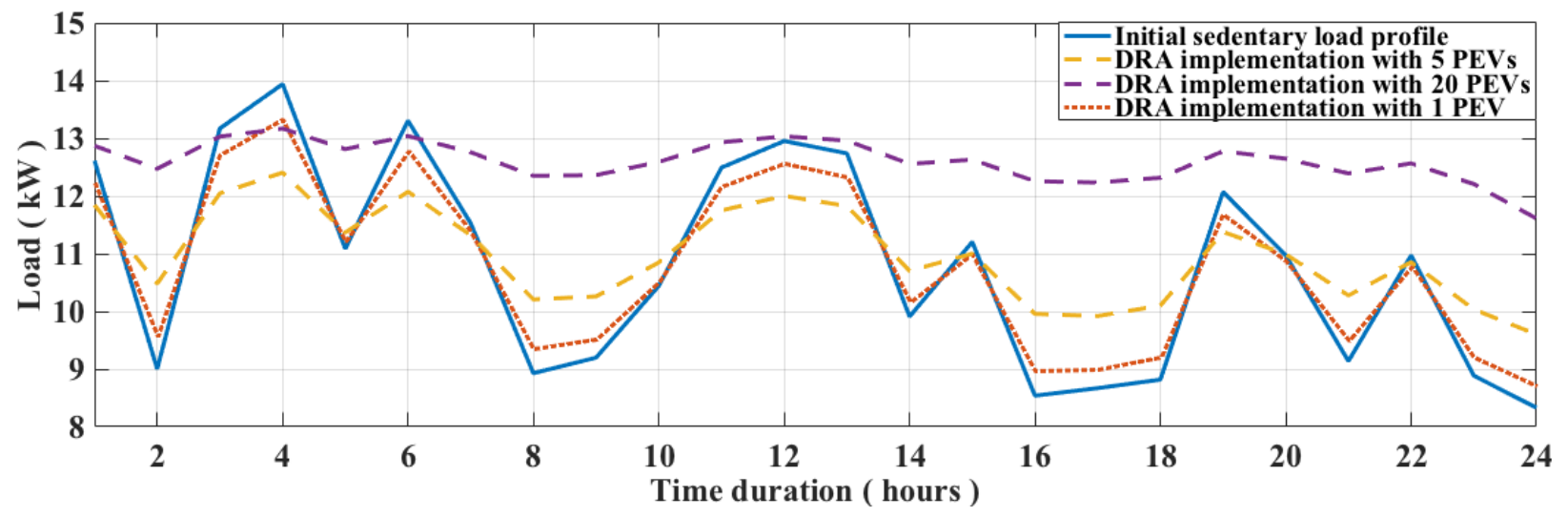

Fig. 3.9 Effect of number of PEVs

Here, the yellow line represents the load profile after implementation of the DRA approach in which the flattening objective is maximized. On the other hand, the violet line represents DRA implementation in which the smoothening objective is preferred.

\subsubsection{Effect of number of interacting PEVs}

The DRA exploits the availability of the total time period along with the lower and upper constraints of individual PEVs which allows PEV to not only be a power sink but also a power source. However, in comparison to the overall power grid load profile, the individual PEV contribution is minuscule due to the comparatively low operational band of $S O C_{\text {downlimit }}$ and $S O C_{\text {uplimit }}$. However, the number of participating PEVs increased their combined contribution can be quantified. In figure 3.9 each plot corresponds to a different number of interacting PEVs aiding the power grid by constructing a smooth and flat load profile. In this case the value of design parameters $\mu$ and 
$\eta$ is assumed to be 0.5 each. It is evident from (3.1) that as the number of PEVs increases, one obtained a comparatively more smooth and flat grid load profile.

\subsection{Conclusions}

In this chapter, the incorporation of PEVs into the grid for overcoming the problem of demandsupply mismatch is addressed. We proposed an output consensus which is an application of DRA for managing distributed integral load of PEVs connected to the power grid. The concept of DRA and passivity approach is feasible for providing active power load and load shifting minimization through a fair scheme of PEV charging. The representative case study results highlight the effectiveness of the proposed approach in grid supply smoothening as well as confirms the desired performance in load management of PEV. The future aim is to conduct a case study considering variation in performance parameters of PEVs participating in grid enhancement activities. 


\section{CHAPTER 4}

\section{Power Flow Analysis using Deep Learning Techniques in a Three Phase Unbalanced Distribution Network}

\subsection{Introduction}

Load flow (LF) analysis is the most important study performed by utility, required in all stages of power system, especially in operation and planning. Some of the load flow techniques used in Transmission systems like Newton Raphson, Gauss-Seidel can not be applied to Distribution systems. Such load flow analysis can be performed using different methods, including iterative power flow analysis. Some of the main iterative power flow methods are backward forward sweep, ladder network, ratio flow methods and few others. To identify the problematic area in the distribution grid and predicting flows based on limited network measurements several traditional algorithms are developed and well documented in the literature. These traditional methods are model based and requires network detailed information. Backbone of these methods are the traditional power flow algorithms. Also, most distribution network states are not available through measurements which gives limited observability of the network and because of that network issues are not identifiable. But advancement in database and deep learning methods made possible predicting unmeasured state utilizing time series data even with limited measurements. In this chapter, we are utilizing deep neural network and its several architecture as a first step to perform three phase unbalanced power flow utilizing offline planning data. For problems containing huge data, deep learning is best suited than traditional neural networks. We have formulated this problem as multi output regression model where two or more output values are predicted based on input. The training and testing data is generated through OpenDSS MATLAB COM interface. The training data is given to neural network $(\mathrm{NN})$ model to train the model. After generating the training dataset, neural network model predict the result for test input data. This proposed method is tested on IEEE 4 node, IEEE 123 node test feeders, and American Electric Power (AEP) feeder model. The results for Radial Basis Function Network (RBFnet), Multi Layer Perceptron (MLP), and Convolutionary Neural Network $(\mathrm{CNN})$ models are discussed in this chapter. The results demonstrate that all the three neural networks predict the load flow quantities with higher accuracy. We also extend 


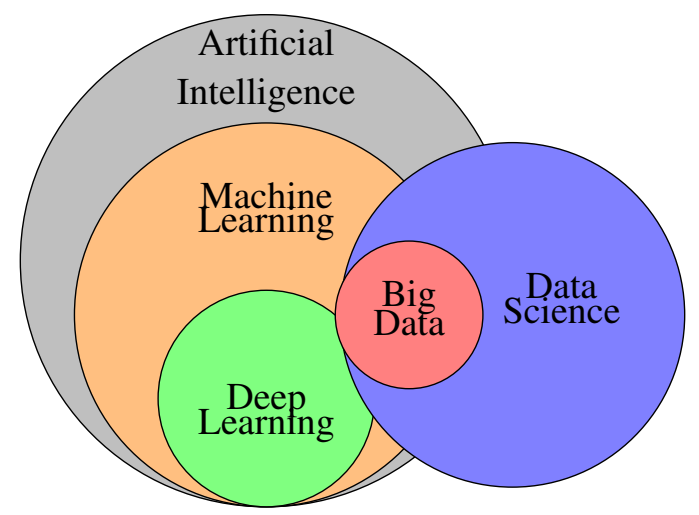

Fig. 4.1 Relation Between Machine Learning and Data Science

this work to predict the power flow for some "unseen" events by the grid operator such as Topology change which is a phenomenon caused by extreme weather events, faults. Also, we integrate renewable energy resources into the grid and predict the power flow values using CNN model.

\subsection{Literature Survey}

Load flow analysis is performed to compute the steady-state operating values of node voltages, line currents, angles, and power losses at a given load. A power utility needs to analyze these variables at a regular interval in order to plan for future in case of some hypothetical critical conditions like system failure or fault analysis. Other important application of load flow analysis is to plan and help in expanding the existing power system. The distribution system is facing extraordinary changes due to addition of distributed energy resources (DERs) like electric vehicles (EVs), wind, solar, distributed generators (DGs), and storage. DERs add to the system challenges like variability, uncertainty in control, infrastructure, and operation of the grid. For such new power system, performing power flow is very important to know the effects of induction of different entities into the grid.

Figure 4.1 depict the interconnection between the different approaches of artificial intelligence (AI) and data science. Machine learning (ML) is one of the subsets of AI where machine learns the objective by accessing the historical data. In ML, programmers do not need to explicitly write the instructions on what action to perform using the data, instead machine acts on the new data using learned pattern from historical data. Deep learning (DL) on the other hand is a subset of machine learning with higher accuracy than other ML methods, uses a larger dataset, and a more complex neural network.

Machine learning (ML) has numerous application in power system data analytics, load forecasting, renewable like wind, solar forecasting, state estimation, fault detection, grid security analysis, and power flow study. Some recent developments in machine learning (especially deep learning) 
plays major role in tackling the increasing burden on the smart power grid due to the uncertainty of distributed energy resources (DERs). One of the most important application is the power flow analysis as utilities need to perform this to analyze the steady-state condition of the network. Different methodologies of machine learning have been used to perform the load flow. [89] investigates the inverse power flow using machine learning models. They compare the results of support vector regression (SVR) and neural network (NN) models. Artificial neural network (ANN) has been used to perform load flow in the Western Systems Coordinating Council (WECC) 9 bus system in [90]. ANN is a machine learning technique which is a standard neural network with one input and output layer and just one hidden layer. In this paper, the author uses a small number of input and output neurons. ANN has also been used to short term load forecasting in [91-93], where previous load history has not been used in the NN model to predict the load. [94] proposed a support vector machine (SVM) is another approach of ML for load forecasting, where the computation time becomes the problem when solving a large scale problem. Also, using SVM for such applications, selection of kernel function is difficult.

$[95,96]$ discuss the short term load forecasting using deep neural network where the author showed that the load forecasting accuracy is enhanced by using DNN. Deep neural network (DNN) or deep learning (DL) is another approach of machine learning. DL is applied in several industrial applications especially image recognition, prediction, audio recognition and many more. DL is an ANN with many hidden layers where consecutive layers are connected with each other but the neurons in the same layer are not connected. With the advancement of smart grid, smart buildings are also emerging to be one of the important factors in green ecosystem. The interconnection of smart grid and green building can cause the power to flow in both directions. In such case, it is very important to know the energy consumption prediction for a large time period. Based on this building level prediction, DERs can be used optimally. [97] proposed supervised and unsupervised energy consumption prediction of industrial and commercial buildings. This is helpful for building owners to plan out the energy consumption over the time.

With the advancement in deep learning, it is proven to be important in solving data-driven problems in power system. In data-driven power system, the main challenge is to protect the network from the data attacks. In ML approaches, a hacker can attack the input data and add some malicious measurements which can compromise the performance of the model, even if it does not have access to the ML model. [98] presents a review on the vulnerability and security of the power system because of machine learning approaches, where they showcased the attack on classifiers and getting false voltage signals. Power system restoration is another important area where deep learning has been used quite extensively. Fault diagnosis using deep learning for power fault diagnosis has been discussed in $[99,100]$. The input data is collected from SCADA measurements. Results were compared for back propagation and deep learning neural network. [101] proposed 
a convolutional neural network classifier for real-time fault localization in electric grid. They use the 4 kernels of 5 by 1 with stride 1 and vector data received from phasor measurement unit (PMU). However, they do not identify the exact location of the fault point. Results were improved compared to [99]. Fault localization is performed for different types of fault and different level of unobservable data. For such unobservable distribution system, [102] proposed state estimation using deep learning. Unobservability arises when sensor data is missing or sensor is at fault or not placed correctly. Bad data were detected and removed before the state estimation. Because of unobservability, some of the prominent network problems are not identifiable. In this work, we are using deep neural network architecture like CNN to predict the power flow by utilizing the available data.

The load flow for distribution system is very complex and traditional iteration based methods may not provide accurate results. In this chapter, we aim to present a trained neural network which predicts the branch currents, node voltages, and voltage and current angles, with high accuracy. Many traditional iterative based power flow methods for distribution system take a lot of time to perform load flow for a large grid and do not converge well for unbalanced system. Also, with two way power flow due to renewable energy sources, demand response, AMIs in smart grid, these traditional method will need a lot of modifications. We propose to replace these traditional iterative based load flow techniques with our trained neural networks. In this work, we present a trained neural network which predicts the branch currents, node voltages, angles, power losses with high accuracy. Sharing the actual model may put the network in danger from malicious attackers. Due to security issues, utilities do not share exact model of distribution system. Our trained deep neural networks can also be used to replace the conventional test cases provided by utilities for research purposes.

We used Radial basis function (RBF) model, multi layer perceptron (MLP), and CNN. RBF network consists of only three layers: the input, hidden, and output layers, popular network in artificial neural network. RBF uses the feedforward propagation. The hidden layer received the input data using a non linear function. The output from RBF takes less time in computation as there is only one hidden layer and activation function in hidden layer calculates the Euclidean distance between the centroid and the input value. MLP network uses the back propagation. MLP is modeled as an interconnected sets of input, hidden, and output layers. MLP model is developed in TensorFlow for our problem. TensorFlow is an open source software library developed by Google. Deep learning, with more number of hidden layers than an ordinary neural network, learns the patterns in available data, used to predict the output. 


\subsection{Distribution Power Flow}

Power flow for distribution system is different than transmission system power flow due to the factors such as:

- High R/X ratio of feeders

- Radial network

- Unbalanced load

Load flow techniques for transmission system like Gauss-Siedel, Newton-Raphson are not efficient and do not converge to the solution for radial distribution system [103]. The forward-backward sweep (FBS) method is one of the most commonly used distribution system power flow (DSLF) methods [104].

$$
\left.\begin{array}{rlrl}
V_{i+1} & =A_{k} V_{i}^{\text {new }}-B_{k} I_{i+1} & & \text { Backward Sweep } \\
V_{i} & =a_{k} V_{i+1}+b_{k} I_{i+1} \\
I_{i} & =d_{k} I_{i+1}
\end{array}\right\} \quad \text { Forward Sweep }
$$

Equations 4.1 and 4.2 show the backward and forward sweep for a component in the distribution systems, where $a_{k}, b_{k}$, and $d_{k}$ represent the $a, b$, and $d$ matrix for $k^{\text {th }}$ component of the system (line, transformer, voltage regulators). The detailed model for Forward backward sweep is mentioned in Table 4.1 for various components. Fast decoupled power flow method is also used for radial distribution power flow [105]. [106] uses bus-injection to branch-current (BIBC) matrix and branch-current to bus-voltage (BCBV) matrix to solve bus voltages iteratively. In this work, we have used an electric power distribution system simulator known as OpenDSS to perform load flow and generate training and testing data (explained in later section). OpenDSS uses the solution of nonlinear admittance equation to analyze the load flow as mentioned [107]: $I(V)=Y_{\text {system }} V$ where $I$ is the injection currents from power conversion elements such as load, PV, storage, and Generators in the circuit and $Y_{\text {system }}$ is the admittance matrix of the system. The solution of this equation found to work well for the distribution network if the initial voltage is close to the final solution.

\subsection{Data Analysis}

In this section, we discuss the characterization of data. The comparison between big data and large data is also analyzed here. 
Table 4.1 FBS summary [108]

\begin{tabular}{|c|c|c|c|}
\hline Component & & Backward Sweep & Forward Sweep \\
\hline $\begin{array}{l}\text { Overhead Lines/ } \\
\text { Underground cables }\end{array}$ & \multicolumn{2}{|c|}{$V_{i}^{\text {new }}=V_{\text {spec }}-Z I_{i}$ for first calculation } & $\begin{array}{l}V_{i}=V_{i+1}+Z I_{i+1} \\
I_{i}=d_{t} I_{i+1}\end{array}$ \\
\hline $\begin{array}{l}\text { Three Phase } \\
\text { Transformer }\end{array}$ & \multicolumn{2}{|c|}{$V_{i+1}=A_{t} V_{i}^{\text {new }}-B_{t} I_{i+1}$} & $\begin{array}{l}V_{i}=a_{t} V_{i+1}+b_{t} I_{i+1} \\
I_{i}=d_{t} I_{i+1}\end{array}$ \\
\hline Switch & \multicolumn{2}{|c|}{$\begin{array}{l}V_{i}^{\text {new }}=K V_{\text {spec }} \\
V_{i+1}=K V_{i}^{\text {new }}\end{array}$} & $\begin{array}{l}V_{i}=K V_{i+1} \\
I_{i}=K I_{i+1}\end{array}$ \\
\hline Capacitor & \multicolumn{3}{|c|}{$\begin{array}{l}Z_{i}^{p h-n}=\frac{\left|V_{i}^{p h-n}\right|^{2}}{S_{i}^{p h-n *}}, I C_{i}^{p h-n}=\frac{V_{i}^{p h-n}}{Z_{i}^{p h-n}} \text { Wye Connected } \\
Z_{i}^{p h-p h}=\frac{\left|V_{i}^{p h-p h}\right|^{2}}{S_{i}^{p h-p h *}}, I C_{i}^{p h-p h}=\frac{V_{i}^{p h-p h}}{Z_{i}^{p h-p h}} \text { Delta Connected }\end{array}$} \\
\hline \multirow{4}{*}{ Load } & Load Type & Wye & Delta \\
\hline & Const PQ & $I L_{i}^{p h}=\left(\frac{S_{i}^{p h-n}}{V_{i}^{p h-n}}\right)^{*}$ & $I L_{i}^{p h}=\left(\frac{S_{i}^{p h-p h}}{V_{i}^{p h-p h}}\right)^{*}$ \\
\hline & Const Z & $\begin{array}{l}Z_{i}^{p h-n}=\frac{\left|V_{i}^{p h-n}\right|^{2}}{S_{i}^{p h-n *}} \\
I L_{i}^{p h-n}=\frac{V_{i}^{p h-n}}{Z_{i}^{p h-n}}\end{array}$ & $\begin{array}{l}Z_{i}^{p h-p h}=\frac{\left|V_{i}^{p h-p h}\right|^{2}}{S_{i}^{p h-p h *}} \\
I L_{i}^{p h-p h}=\frac{V_{i}^{p h-p h}}{Z_{i}^{p h-p h}}\end{array}$ \\
\hline & Const I & $\begin{array}{l}I L_{i}^{p h-n}=\left|I L_{i}^{p h-n}\right|^{*} \\
\angle \delta_{i}^{p h-n}-\theta_{i}^{p h-n}\end{array}$ & $\begin{array}{l}Z_{i}^{p h-p h}=\frac{\left|V_{i}^{p h-p h}\right|^{2}}{S_{i}^{p h-p h *}} \\
I C_{i}^{p h-p h}=\frac{V_{i}^{p h-p h}}{Z_{i}^{p h-p h}}\end{array}$ \\
\hline Distributed Load & \multicolumn{3}{|c|}{$\begin{array}{l}\text { 1. Two-thirds load is lumped at one-fourth length of line } \\
\text { from sending end } \\
\text { 2. One-third load is lumped at receiving node. }\end{array}$} \\
\hline
\end{tabular}

\subsubsection{Big Data in Power System}

Figure 4.2 depicts the different types of big data in the context of the power system. Big data can not be referred just as a collection of huge data, also it must possess four V's [109]; Velocity, Variety, Volume, and Value.

- Volume:- refers to the large scale data, may start from gigabytes and more. Weather data, Advanced metering infrastructure (AMI) data can be of such size. Utility measurements like phasor measurement units (PMU), customer response for pricing signals, demand response using smart meters are sources of data flowing in gigabytes. PMU is used to measure the magnitude and angle of voltage and currents. System restoration after faults, severe events can be faster with such data analysis.

- Velocity:- Data coming in continuous form from Phase measurement unit (PMUs), Supervisory control and data acquisition (SCADA). Velocity also refers to the frequency or time intervals of data collected. Market data can also be an example of possessing velocity in data. 


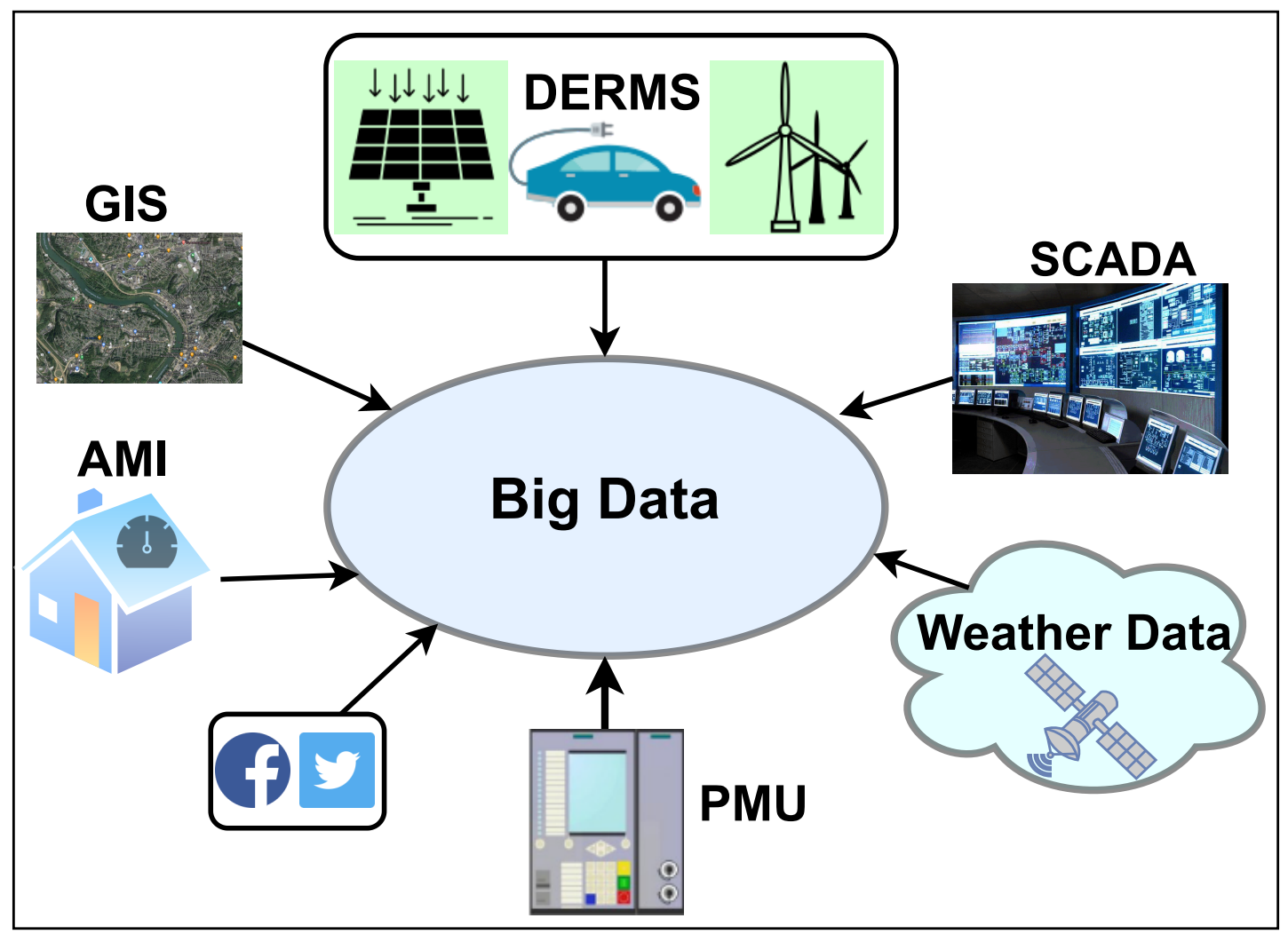

Fig. 4.2 Big Data in Power System

- Variety:- Structured and unstructured data are different varieties of big data. In a power system, data coming from sensors and meters are termed as structured data, while customer data, weather forecast data, meter IDs are examples of unstructured data. As shown in figure 4.2, distributed energy resources along with energy management system and distribution management system use a variety of customer data, weather data, market pricing data for the complete monitoring and control of power grid.

- Value:- The value of collected data which is helpful for creating knowledge like stochastic, events. Using big data analytics and machine learning techniques, power system data can be processed to gain the valuable information and use that to form the knowledge.

\subsubsection{Data Preprocessing}

Before we feed the raw data to our model, to achieve better results, data has to be formatted and convert it as structured data. Some of the steps for data preprocessing in machine learning includes [110] 
- Data Cleaning: Due to some human or machine error, model may miss some of the learning data. Before we proceed with such faulty, incomplete data, it is important to take care of missing data to avoid the inaccurate learning of model. Cleaning of the data is also important if there is a large amount of duplicate or unwanted data.

- Quality Assessment: Data type might be different in some cases. Some dataset may have inconsistent values if two or more datasets are concatenated.

- Data Transformation: Aggregation of data is useful when data is scattered as machine learning produce better results with a unified data. Normalization plays an important role in machine learning. The aim of the normalization is to rescale data and bring it in the a common range for all the variables.

- Data Reduction: Some datasets that are used in speech recognition, computer vision applications may have a large number of features. Some of these features can be removed without loosing the general information about the datasets, could result in speeding the process.

To understand the normalization, consider the example of power flow data. The voltages, currents magnitude and angle are not in same range so our neural network may not be accurate if fed such unprocessed data. For this purpose, we use min-max normalization. The formula for this type of normalization is given below-

$$
X_{n o r}=\frac{\left(X-X_{\min }\right)}{\left(X_{\max }-X_{\min }\right)}(B-A)+A
$$

where $X_{n o r}$ is the normalized value. $X_{\min }$ and $X_{\max }$ represent the minimum and maximum of the particular variable. $[\mathrm{A}, \mathrm{B}]$ is the range for which dataset is to be normalized. In our problem, we are normalizing in the range of $[0,1]$.

\subsection{Proposed Methodology}

Methodology to use deep learning for load flow prediction is proposed in this section. The schematic diagram of our proposed technique is shown in figure 4.3. Any machine learning prediction approach includes two steps 1) Training 2) Prediction. The distribution grid is modeled in OpenDSS software. OpenDSS is an an open source distribution system simulation tool developed by the Electric Power Research Institute (EPRI) [111]. OpenDSS just need a text file with the distribution network topology to simulate the system. Using COM interface, OpenDSS can interchange data with other tools like MATLAB and provides better flexibility to users to modify the source code and perform the objective. OpenDSS is equipped to simulate the DER grid integration thus helpful in grid modernization. 


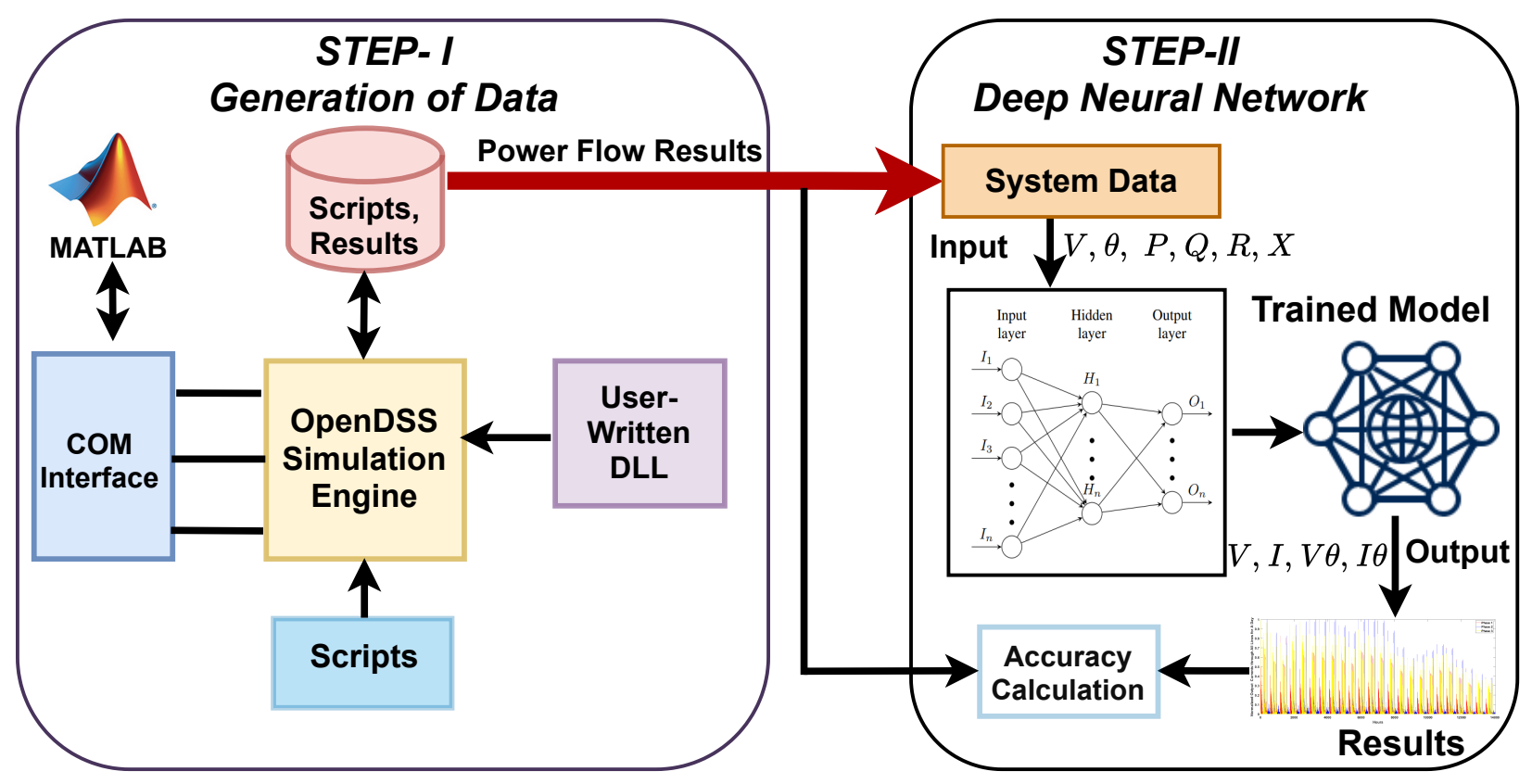

Fig. 4.3 Outline of Proposed Methodology

In this work, training data is generated by load flow analysis using OpenDSS - MATLAB COM interface for our test cases. The input variables which are used to model the grid are source voltage amplitude and angle, line parameters, load active and reactive powers, and loadshapes to perform quasi static time-series simulations. The problem architecture on a broader level is shown is figure 4.4. The generated data set from OpenDSS MATLAB COM interface is the power flow quantities like node voltages, branch currents, voltage and current angles. These values along with the source voltage, line parameters, and the loadshape are fed to neural network model for training the model using learning algorithm. The training data is normalized before it is sent to train the model as all the bus voltages and currents differ in range as described in section 4.4.2. After training, some part of the training data set is used as a test data and again passed on to NN model to get the prediction results. Prediction accuracy is calculated then, which is ultimately minimized.

There are many metrics to evaluate accuracy of the DL algorigthm like classification metrics (accuracy, precision), statistical metrics, ranking metrics, logarithmic loss, R2 score, regression metrics. In this work, we have evaluated the accuracy using two regression metrics mentioned below:

- Mean Absolute Error (MAE)- measures the average of absolute errors in the predicted variables.

$$
M A E=\frac{1}{n} \sum_{i=1}^{n}\left|y_{i}-\hat{y}_{i}\right|
$$

From equation 4.4, it is clear that MAE is the mean error between predicted and actual values 


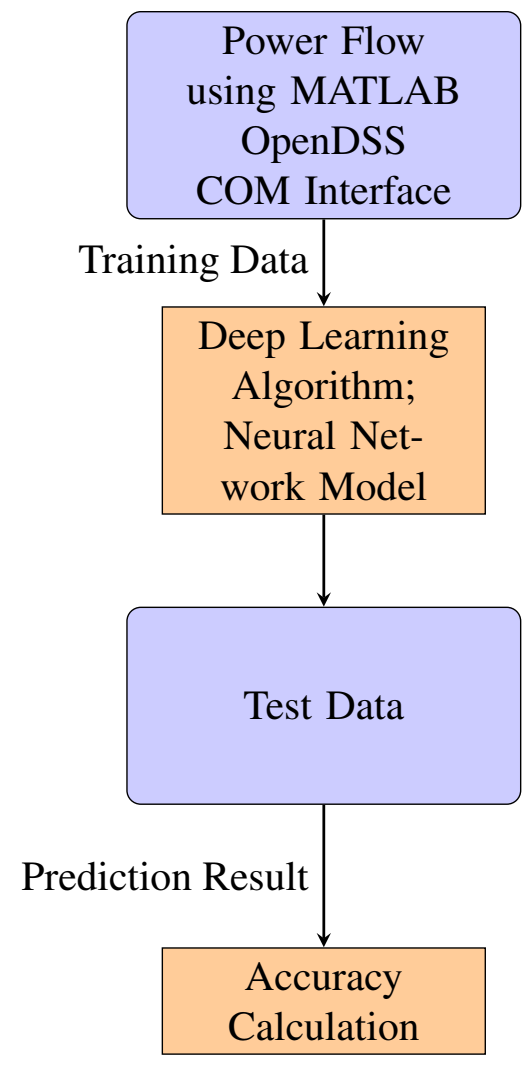

Fig. 4.4 Problem Architecture

in the dataset considering $n$ predictions were generated.

- Mean Squared Error (MSE)- As the name suggest of this accuracy metric, it is a mean of the square of the errors.

$$
M A E=\frac{1}{n} \sum_{i=1}^{n}\left(y_{i}-\hat{y}_{i}\right)^{2}
$$

\subsection{Neural Network Model}

We have utilized 3 different neural network model for our proposed method: (1) multi-layer perceptron (MLP); (2) convolutional neural network (CNN); (3) radial-basis function (RBF) network. In this section, we discuss the general details for all three neural network models and the specific implementation details for our proposed method have been provided in result section.

\subsubsection{Multi-layer Perceptron}

A perceptron or a neuron is a linear classifier i.e., it is an algorithm that classifies input by separating two categories with a straight line. Input is typically a feature vector $x$, which is multiplied 


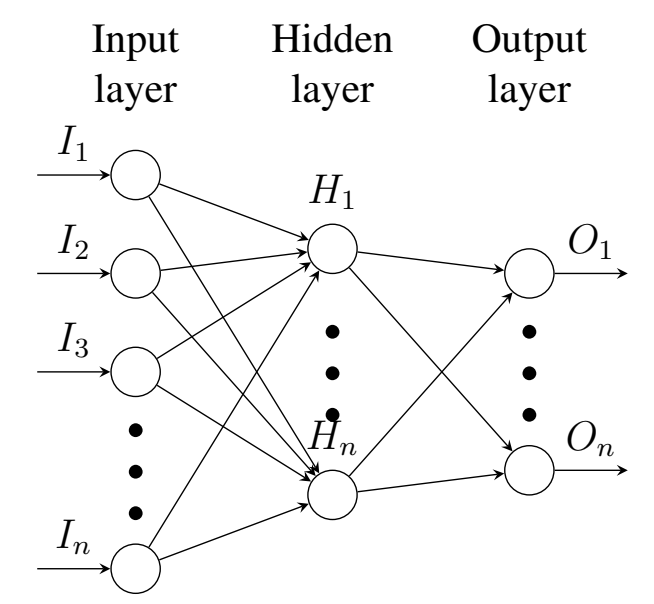

Fig. 4.5 Multi Layer Perceptron

by weights $w$ and added to a bias $b: y=w x+b$. A perceptron produces a single output based on several real-valued inputs by forming a linear combination using its input weights (and sometimes passing the output through a nonlinear activation function). It is given as:

$$
y=\phi\left(\sum_{i=1}^{n} w_{i} x_{i}+b\right)=\phi\left(\mathbf{w}^{T} \mathbf{x}+b\right),
$$

where $\mathbf{w}$ denotes the vector of weights, $\mathbf{x}$ is the vector of inputs, $\mathrm{b}$ is the bias and $\phi$ is the non-linear activation function.

A multi-layer perceptron (MLP) is a deep, artificial neural network, which is composed of more than one neuron. It is composed of an input layer that receives the input, an output layer that makes a decision or prediction about the input, and in between those two, an arbitrary number of hidden layers that are the true computational engine of the MLP. Each hidden layer consists of a set of neurons and each neuron is fully connected to all neurons in the previous layer, and where neurons in a single layer function completely independently and do not share any connections. A general architecture for MLP is shown in figure 4.5. MLPs are often applied to supervised learning problems: they train on a set of input-output pairs and learn to model the correlation (or dependencies) between those inputs and outputs. Training involves adjusting the weights and biases, of the model in order to minimize error. Backpropagation is used to make those weight and bias adjustments relative to the error, and the error itself can be measured in a variety of ways, including by root mean squared error (RMSE).

MLP is a feed-forward network that consists of two motions: forward pass and a backward pass. In the forward pass, the input moves from the input layer through the hidden layer to the output layer, where a decision is made by the output layer. An error is calculated by comparing the decision at the output layer against the ground truth labels. 
In the backward pass, partial derivatives of the error function concerning weights and the biases are calculated and back-propagated through the MLP. Differentiation gives us the gradient or the landscape of error, to adjust the parameters of the MLP so as to move it one step closer to the minimum error. This can be done using any kind of gradient-based optimization such as stochastic gradient descent (SGD). This forward and backward pass are repeated till the error can gono lower, which is known as convergence.

\subsubsection{Convolutional Neural Network}

Convolutional neural networks (CNN) [112] are biologically inspired models that are analogous to the connectivity pattern of neurons in the human brain. Convolutional Neural Networks are very similar to MLPs: they are made up of perceptrons (neurons) that have learnable weights and biases. Each neuron receives an input, performs a dot product and optionally follows it with a non-linearity. The whole network still expresses a single differentiable score function: from the raw image pixels on one end to class scores at the other. And they still have a loss function (e.g. SVM/Softmax) on the last (fully-connected) layer. However, the major difference between the CNN and MLP is that the CNNs are majorly used for inputs of image type, due to which the forward function is much easier to implement with less number of parameters in the network. The role of the $\mathrm{CNN}$ is to reduce the images into a form which is easier to process, without losing features which are critical for getting a good prediction.

CNN consists of three basic components: convolution, pooling and fully connected layers. In the convolution layer one tries to learn a filter bank given input feature maps. The input of a convolution layer is in the form of a $n_{1} \times n_{2} \times d$, where $d$ denotes the number of two-dimensional feature maps of size $n_{1} \times n_{2}$ in the input. Let $x_{i}(j, k)$ denote the component at row $j$ and column $k$ in the $i$ th feature map, and we use $x_{i}^{l}$ to denote the complete $i$ th feature map at layer $l$. If one want to learn $f$ set of filters of size $k_{1} \times k_{2}$, the output $x_{(l+1)}$ for the next layer will still be a three-dimensional array with $f$ number of two-dimensional feature maps of size $\left(n_{1}-k_{1}+1\right) \times$ $\left(n_{2}-k_{2}+1\right)$. More formally, the convolution layer computes the following:

$$
x_{j}^{(l+1)}=s\left(\sum_{i} K_{i j} * x_{i}^{(l)}+b_{j}\right),
$$

where $K_{i j}$ denotes the filter that connects feature map $x_{i}^{l}$ to output map $x_{j}, b_{j}$ is the bias for the $j$ th output feature map, $s($.$) is some elementwise non-linearity function and *$ denotes the discrete two-dimensional convolution. We denote $j$ th convolutional layer with $f$ filters of size $k_{1} \times k_{2}$ by $C_{\left(f, k_{1} \times k_{2}\right)}^{(j)}$.

It is very common to insert a pooling layer in between the convolutional layers in a CNN. The pooling layer is responsible for reducing the spatial size of the output of the convolutional layer 
to reduce the amount of parameters and computation in the network, and hence to also control over-fitting. Furthermore, pooling layer is useful for extracting dominant features that are position and rotation invariant, thus maintaining the process of effectively training of the model.

Pooling layer operates independently on every feature map (output of convolutional layer) and resizes it spatially, using the max or average operation. For example, with a pooling filter size of $m_{1} \times m_{2}$ an input with $f$ number of two-dimensional feature maps of size $n_{1} \times n_{2}$ will result an output of $f$ number of feature maps of size $n_{1} / m_{1} \times n_{2} / m_{2}$. For average (mean) pooling, the output will be the average of all the values from the portion of the image covered by the filter, and for max pooling the output will be the maximum value from the portion of the image covered by the filter. We denote the $j$ th pooling layer with pooling filter size of $m_{1} \times m_{2}$ as $P_{m_{1} \times m_{2}}^{(j)}$.

The convolutional layer and the pooling layer, together are considered to be the i-th layer of a CNN. Depending on the application requirements and complexities in the images, the number of such layers can be adjusted for acquiring the required low-levels details. The output of the last pooling layer is flattened and fed to the fully connected layer for further processing. A fullyconnected layer is usually added to a $\mathrm{CNN}$ to learn non-linear combinations of the high-level features generated at the output of the convolutional or pooling layer.

Neurons in a fully connected layer have full connections to all activations in the previous layer as seen in MLPs. Their activations can hence be computed with a matrix multiplication followed by a bias offset as given in (4.6). We denote the $j$ th fully connected with $h$ neurons or hidden units as $F_{h}^{(j)}$

Generally, a CNN consists several stacks of convolution and pooling layers followed by a few fully-connected layers. The last fully-connected layer is usually associated with some loss function to provide training signals and the backpropagation applied to every iteration of training by using gradient descent. For example, in classification the last layer is normally a softmax layer, and over a series of epochs, the model is able to distinguish between dominating and certain low-level features in images and classify them using the softmax classification technique with cross-entropy loss.

\subsubsection{Activation Function}

In the above sections on CNN and MLP, we have discussed the basic architecture of a CNN and MLP. However, in order to understand how the neural networks (CNN and MLP) are able to solve the non-linear complex problems, it is very important to understand the activation functions and their importance. Activation functions are mathematical functions that determine the output of a neural network. The activation function is attached to each neuron in the network, and determines whether the neuron should be activated or not, based on the neuron's input.

Basically, in a neural network, input data points (numeric) are fed into the neurons in the input 


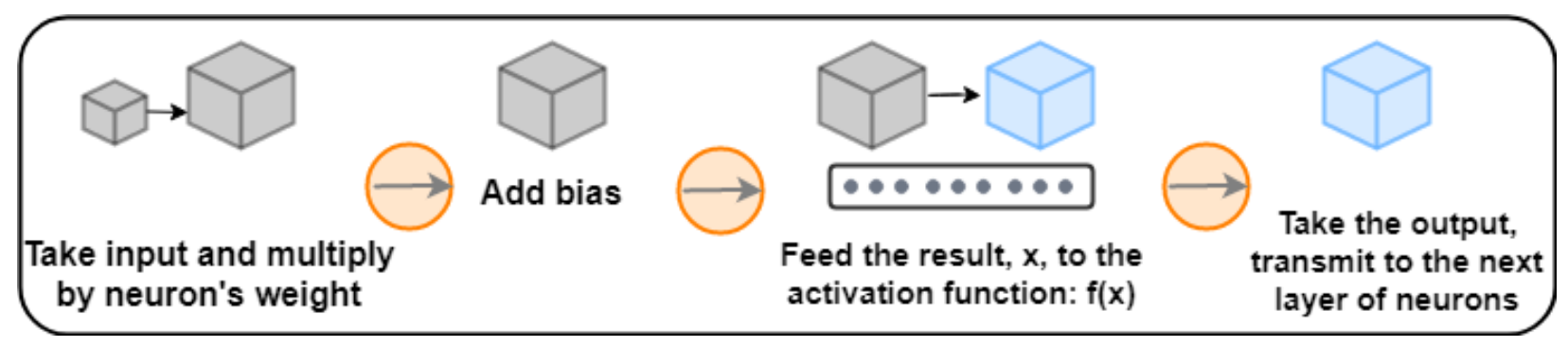

Fig. 4.6 Basic process of a neuron

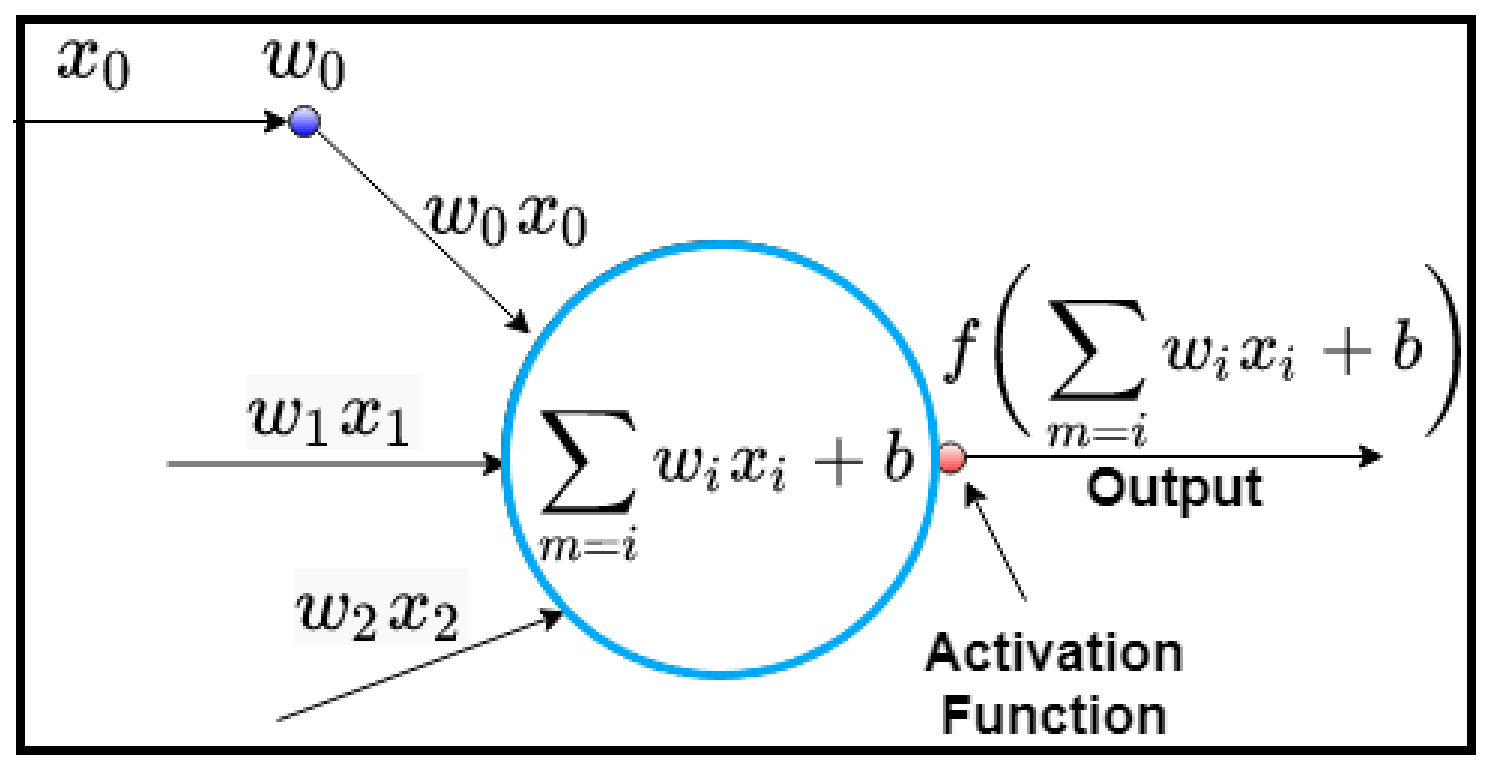

Fig. 4.7 Mathematical model of a biological neuron

layer. Each neuron has a weight, and multiplying the input data point with the weight gives the output of the neuron, which is transferred to the next layer shown in figure 4.6. The activation function can be considered to be a mathematical "gate" in between the output of the neuron and the input being fed to the next layer as shown in figure 4.7. Imagine a neural network without the activation functions. In that case, every neuron will only be performing a linear transformation on the inputs using the weights and biases. Although linear transformations make the neural network simpler, but this network would be less powerful and will not be able to learn the complex patterns from the data [113]. Activation functions also help normalize the output of each neuron to a range between 1 and 0 or between -1 and 1 . Another important aspect of activation functions is that they must be computationally efficient as they are calculated across a large number of neurons for each data sample

An activation function can be as simple as a step function that turns the neuron output on and off, depending on a rule or threshold. An activation function can also be a linear function, 
which takes the inputs, multiplied by the weights for each neuron, and creates an output signal proportional to the input. However, we would like discuss more about the non-linear activations:

1. Sigmoid: It is one of the most widely used non-linear activation function. Sigmoid transforms the values between the range 0 and 1 . The mathematical expression for sigmoid is

$$
f(x)=\frac{1}{\left(1+e^{-x}\right)}
$$

2. Tanh: The tanh function is very similar to the sigmoid function with the only difference being that Tanh is symmetric around the origin. The range of values in this case is from - 1 to 1. Thus the inputs to the next layers will not always be of the same sign. The tanh function is defined as:

$$
f(x)=\frac{2}{\left(1+e^{-2 x}\right)-1}
$$

3. ReLU: ReLU stands for Rectified Linear Unit. The ReLU activation function is a widely used activation function, especially with CNNs and has gained a lot of popularity in the deep learning domain. The ReLu activation is defined as:

$$
f(x)=\max (0, x)
$$

As seen from the definition of ReLU, it does not activate all the neurons at the same time. For the negative input values, the result is zero, that means the neuron does not get activated. This implies that ReLU activation is easy to compute and does not saturate and does not cause the vanishing gradient problem. Since only a certain number of neurons are activated, the ReLU function is far more computationally efficient when compared to the sigmoid and tanh function. However, ReLu suffers from “dying ReLU” problem. As seen from the ReLu definition, the output is zero for all negative inputs, which means that some nodes may completely die and not learn anything.

4. Leaky ReLu and Parametric ReLU: To overcome this "dying ReLU" problem, Leaky ReLU and Parametric ReLU have been defined in the literature. Instead of defining the Relu function as 0 for negative values of $x$, we define it as an extremely small linear component of $x$. The Leaky ReLU is mathematically defined as

$$
f(x)=\max (0, \alpha x)
$$

Here $\alpha$ is a hyperparameter generally set to 0.01 . As is clear from the defintion, Leaky ReLU solves the "dying ReLU" problem to some extent. However, if we set $\alpha=1$, then Leaky 
ReLU will become a linear function $f(x)=x$ and therefore, will be of no use. Hence, the value of $\alpha$ should never be set close to 1 . If we set $\alpha$ as a hyperparameter for each neuron separately, we get parametric ReLU or PReLU.

5. Swish: Swish is a new, self-gated activation function discovered by researchers at Google. According to their paper [114], it performs better than ReLU with a similar level of computational efficiency. the mathematical definition of Swish is given as :

$$
\begin{gathered}
f(x)=x \times \operatorname{sigmoid}(x) \\
f(x)=\frac{x}{\left(1+e^{-x}\right)}
\end{gathered}
$$

For our experiments, we have used ReLU activation as the network converges faster and is computationally efficient when compared to other activation functions.

Epochs vs Iteration: In neural network, it is difficult to pass entire training datasets through the model at once, so we need to divide the dataset into some batches. Once all these batches are passed forward and backward through the network for one cycle, it is called epoch. Neural network needs to adjust the weights and recalculate again and again to effectively learn the pattern of input training data. Which is why we need to use many epochs. For example, if we have 1000 training data points that we need to pass for model to learn. It is too big to feed so we divide into 200 number of batches. Since batch size is 200 , to complete an epoch, we will need 5 iterations.

\subsubsection{Radial Basis Function Network}

Radial basis function networks (RBFN) are used for exactly this scenario: regression or function approximation. RBFN can be used to model an underlying trend or a function that is represented by given data. RBFN can learn to approximate the underlying trend using many Gaussians (bell) curves.

An RBFN resembles a 3-layer MLP network and consists of an input layer, hidden layer made of radial basis function (RBF) neurons and an output layer with one node per category or class of data. General RBFN architecture is shown in figure 4.8. The input vector, which is an ndimensional vector to be classified forms the input layer. The entire input vector is passed through each RBF neuron. A "prototype" vector, which is one of the vectors from the training set is stored in each RBF neuron. The input vector is compared against the prototype in each RBF neuron resulting in an output value between 0 and 1 which is a measure of similarity. If the input is equal to the prototype, then the output of that RBF neuron will be 1 . As the distance between the input and prototype grows, the response falls off exponentially towards 0 . There are different function 


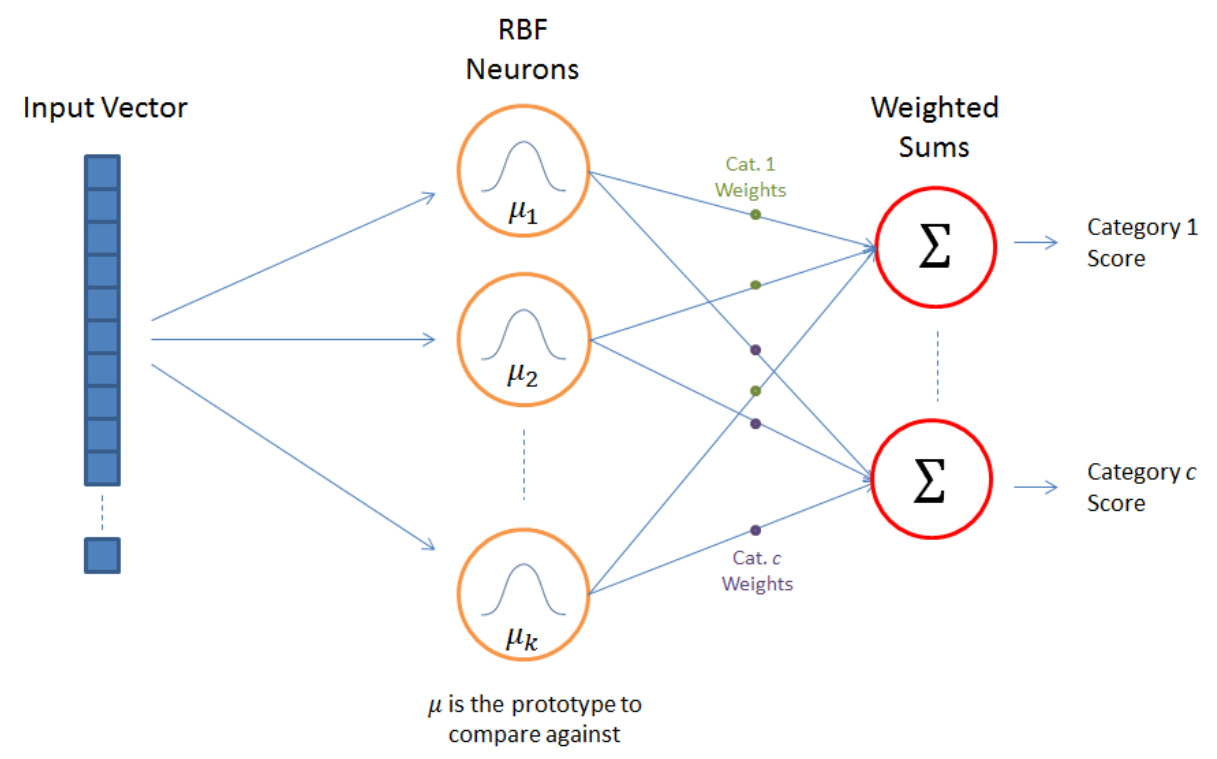

Fig. 4.8 Radial Basis Function Network

that could be used to measure the similarity. However, for RBF neuron activation, we use the RBF function given as:

$$
f(x)=e^{\frac{-(x-\mu)^{2}}{2 \sigma^{2}}} .
$$

In an RBFN, the most commonly used similarity or activation function is RBF, which is based on Gaussian distribution. The Gaussian distribution is given by:

$$
f(x)=\frac{1}{\sigma \sqrt{2 \pi}} e^{\frac{-(x-\mu)^{2}}{2 \sigma^{2}}},
$$

where $\mathrm{x}$ is the input, $\mu$ is the mean and $\sigma$ is the standard deviation. The output layer consists of a set of nodes, one per category that we are trying to classify. Each output node computes a sort of score for the associated category. A weighted sum of the activation values from every RBF neuron is taken to compute the category score. By weighted sum, we mean that each RBF neuron is associated with a weight value by the output node and this weight value is multiplied with the neuron's activation before adding it to the total response. Every output node has its own set of weights and the output node will typically give a positive weight to the RBF neurons that belong to its category, and a negative weight to the others.

To summarize, given an input $x$, an RBF network produces a weighted sum output:

$$
F(x)=\sum_{j=1}^{k} w_{j} \varphi_{j}\left(x, c_{j}\right)+b,
$$




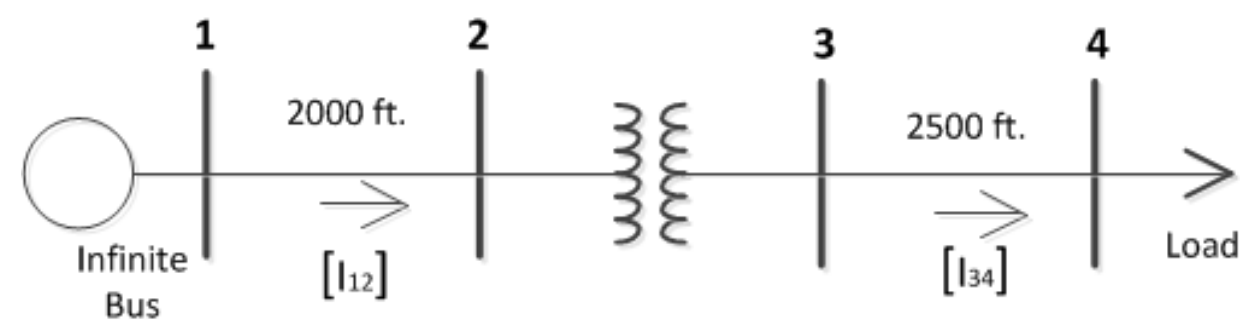

Fig. 4.9 IEEE 4 Node Test Model

where $w_{j}$ are the weights, $\mathbf{b}$ is the bias, $\mathbf{k}$ is the number of bases/clusters/centers, and $\varphi_{j}(\cdot)$ is the Gaussian RBF:

$$
\varphi_{j}\left(x, c_{j}\right)=\exp \left(\frac{-\left\|x-c_{j}\right\|^{2}}{2 \sigma_{j}^{2}}\right) .
$$

The centers of the RBF neurons can be obtained in different ways as long as the entire data space is well represented. The different ways are:

- They can be chosen randomly from the training dataset.

- The centers could be selected using unsupervised learning technique such as K-means clustering [115].

- The centers could also be obtained through supervised learning.

\subsection{Simulation Results}

In this section, we discuss the experimental results of 3 phase unbalanced distribution power flow prediction using our proposed methodology.

\subsubsection{Case 1: IEEE 4 Node Test Case}

Figure 4.9 depicts the IEEE 4 node distribution network, used for implementing the proposed deep learning methodology. Figure 4.10 refers to the loadshape of the load at node 4 . For the IEEE 4 node distribution network, we have generated the data for 3 years using the OpenDSS platform. Therefore the total data points was equal 26280. We have randomly selected 21000 points for training of the neural network (NN) and 5280 points for testing and analysis.

Considering the single load IEEE 4 node distribution network, the input vector dimension for the $\mathrm{NN}$ is equal to 43 and the number of classes or the outputs is equal to 55. The input data consist of voltage source amplitude and angles, line parameters like resistance and reactance, load data, and loadshapes, while the voltage amplitudes, angles, and current amplitudes and angles form the output classes. 


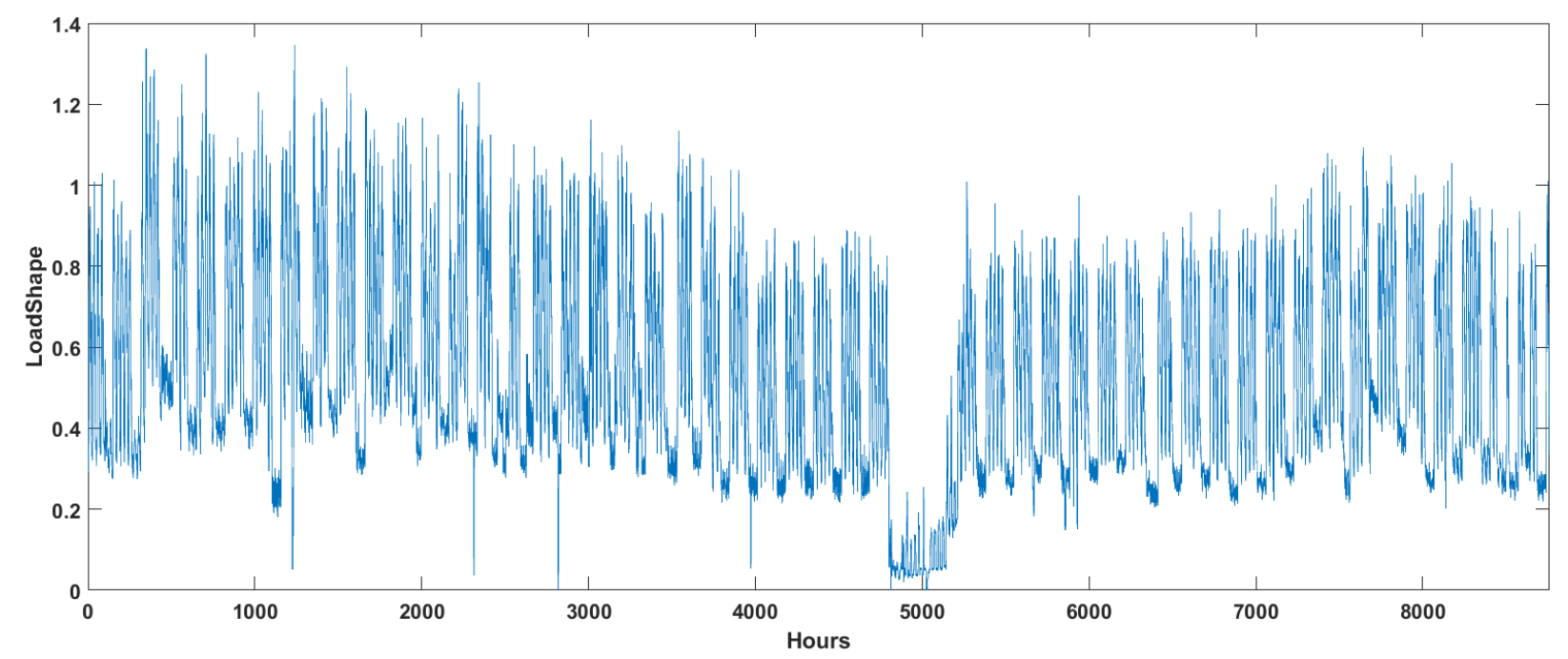

Fig. 4.10 Loadshape

We have used three different neural network models. The implementation and the network parameters for each network model are given below:

1. Convolutional neural network: To use data as input for the CNN model, the input vector size is reduce to 42 by discarding sparse values from the input data. The reduced input vector of size 42 is reshaped into $7 \times 6$ to make it possible to be used as input for CNN. We just use a single layer of input vector $7 \times 6$ Instead of the traditional three-layer input for $\mathrm{CNN}$. The training is carried out by optimizing the mean square error (MSE) objective using mini-batch gradient descent with momentum.

The architecture that we used for $\mathrm{CNN}$ is as follows: two convolutional layers of 32 filters of size $2 \times 2$ and 64 filters of size $2 \times 2$. We have used max pooling layer of size $2 \times 2$ only for the first convolutional layer. The convolutional and pooling layers are followed by four fully connected layers of size 512,256,128, and 64 in that order and finally the output layer of size 54. We use rectified linear unit (ReLU) activation function for all layers except the last layer. We have not used any activation for the last layer. We have minimized the mean square error (MSE) loss which is typically used for regression analysis. The batch size was set to 256 with the learning rate equal to 0.0001 . We have trained the CNN for 1000 epochs.

2. Multi-layer perceptron : For MLP, the size of the input vector is equal to 43 with no value being discarded. For the MLP model, we have used four hidden fully connected layers of size $512,256,128$, and 64 in that order. The output layer is of size 54 . The rest of the training details are consistent with the CNN model except the number of training epochs. The MLP model has been trained for 100 epochs. 
3. Radial-basis function network: Similar to MLP, even for the RBFN model, the size of the input vector is equal to 43 . We have used only one hidden RBF layer. The number of RBF neurons in the hidden layer is equal to 50 . We have used $\mathrm{K}$-means clustering for calculating the centers of the RBF neurons. We have minimized the mean square error loss for the output layer. The batch size was set to 1 with the learning rate equal to 0.0001 . We have trained the RBFN for 150 epochs.

\subsubsection{Case 2: AEP Distribution System}

The AEP feeder 1 shown in figure 4.11, represents a large distribution system provided by american electric power utility for study purposes. Such big feeder is very important in generating a huge data set for deep learning approaches. For the AEP distribution network, we have generated the data for 1 year using the OpenDSS platform. We have total 8760 data points refer to total hours in a year. We have randomly selected 7200 points for training of the neural network (NN) and 1560 points for testing and analysis. Considering the AEP distribution network, the input vector dimension for the NN is equal to 2809 and the number of classes or the outputs is equal to 5880 .

The implementation and the network parameters for each NN model are given below:

1. Convolutional neural network: For the $\mathrm{CNN}$ model training and testing, the input vector of size 2809 is reshaped into $53 \times 53$ to make it possible to be used as input for CNN. The training is carried out by optimizing the mean square error (MSE) objective using mini-batch gradient descent with momentum.

We used two convolutional layers of 64 filters of size $2 \times 2$, and 128 filters of size $2 \times 2$, followed by two more convolutional layers with 256 filters of size $2 \times 2$. We have used max pooling layer of size $2 \times 2$ for all the convolutional layers. The convolutional and pooling layers are followed by four fully connected layers of size 4096, 2048, 2048, and 1024 in that order and finally the output layer of size 5880. Like previous case, we used rectified linear unit (ReLU) activation function for all layers except the output layer. We minimized the MSE error, while Adam optimizer [116] with momentum as 0.5 is selected. The batch size was set to 48 with the learning rate equal to 0.0008 . We have trained the CNN for 1200 epochs. The CNN sequence for the AEP distribution is shown in figure 4.12. For clarity reasons, all the connections of the fully connected layers have not been shown. The black dashed line indicates that there are more convolutional layers, but are not shown due to space issues.

2. Multi-layer perceptron : For MLP, the size of the input vector is equal to 2809 with no value being discarded. For the MLP model, we have used five hidden fully connected layers of size $4096,2048,2048,1024$, and 1024 in that order. The output layer is of size 5880 . We have 


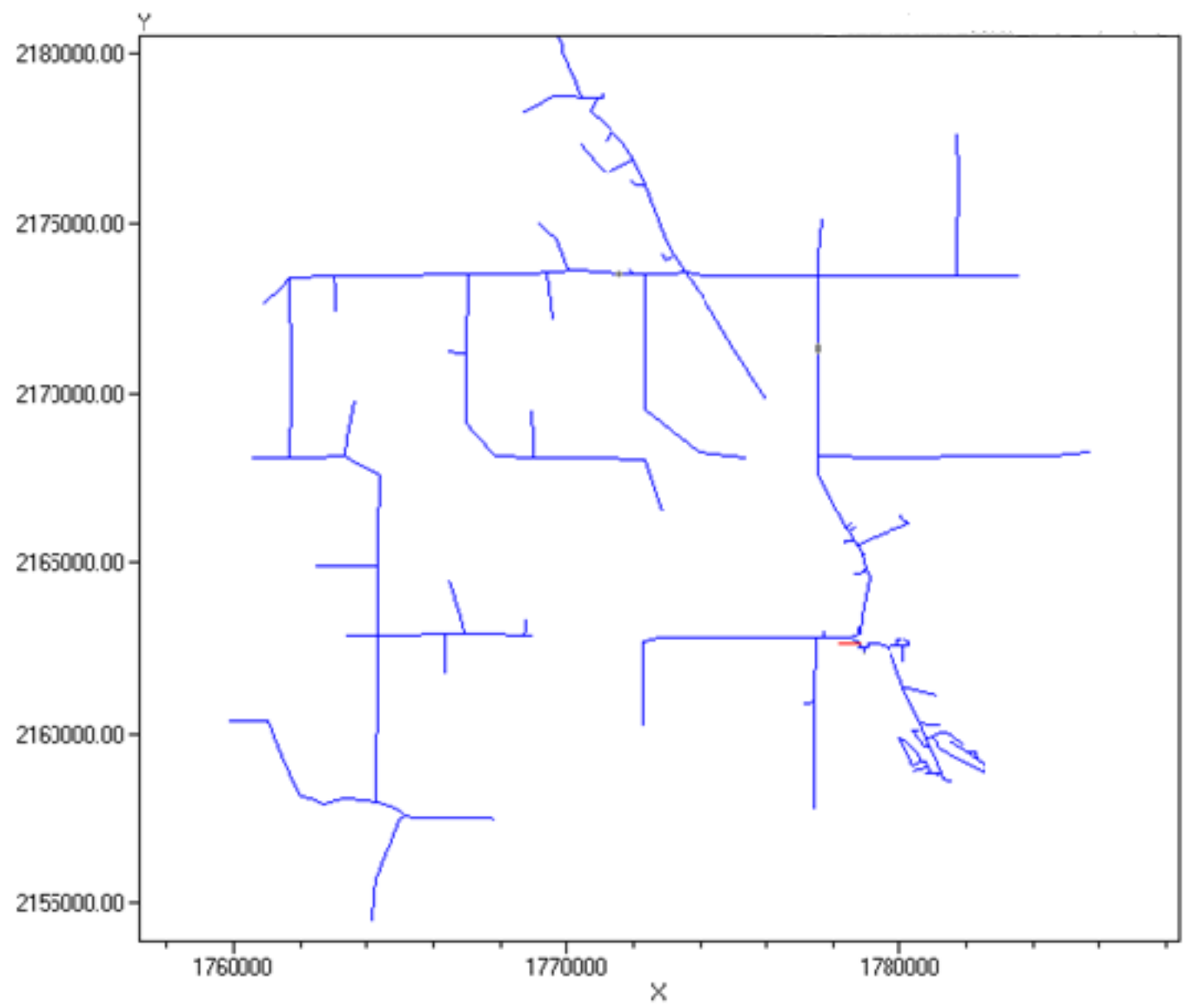

Fig. 4.11 AEP Feeder 1

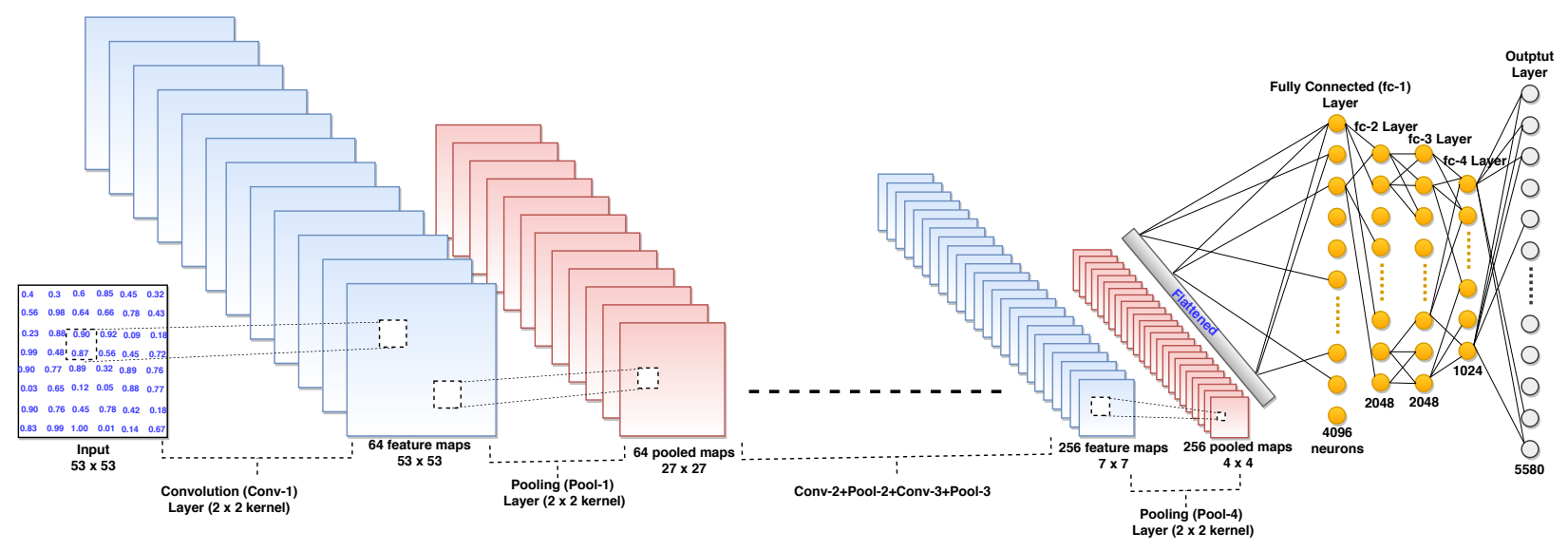

Fig. 4.12 CNN sequence for AEP feeder

used Adam optimizer [116] with momentum as 0.5. The batch size was set to 96 with the learning rate equal to 0.0008 . We have trained the MLP for 1200 epochs. TensorFlow is used 


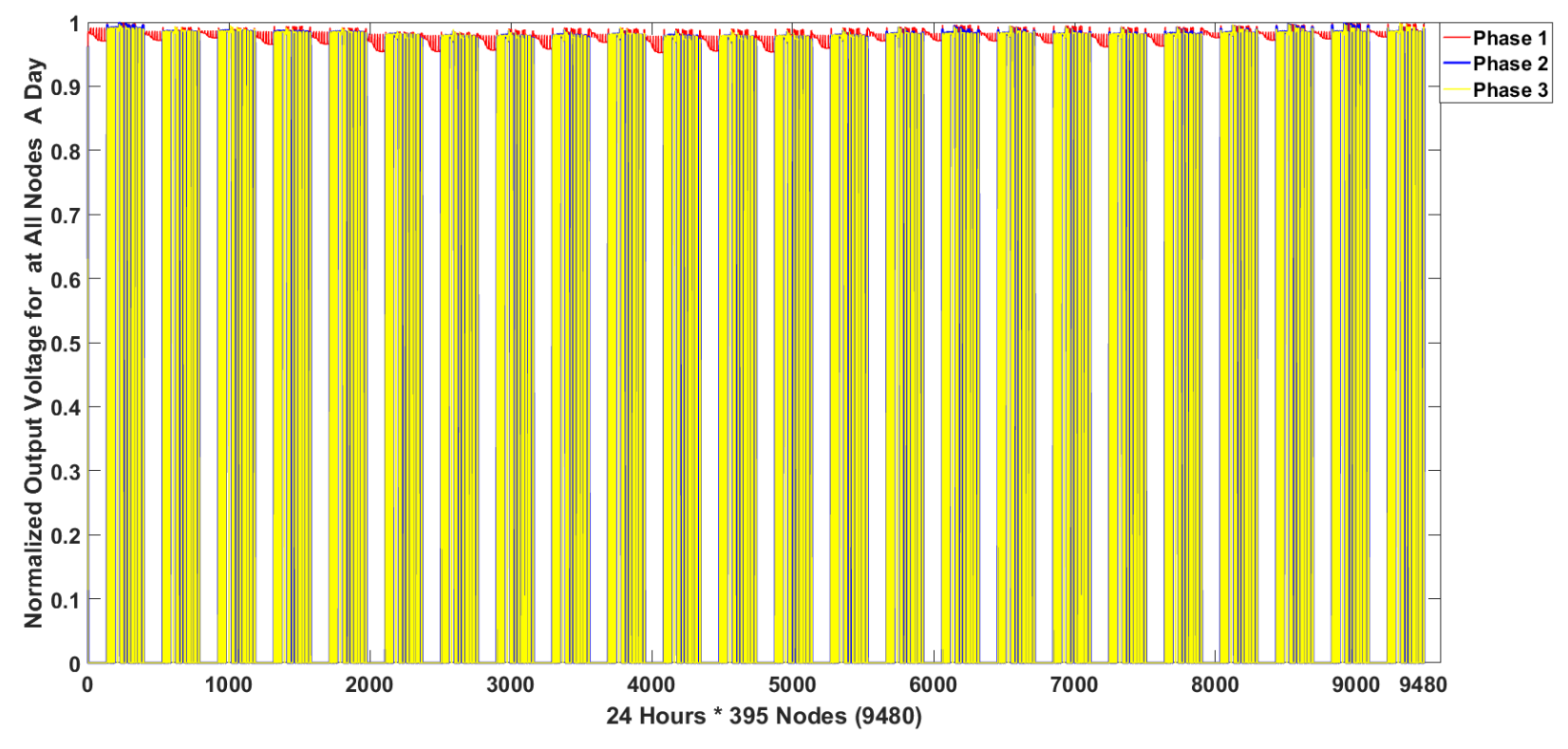

Fig. 4.13 Normalized Output Voltage

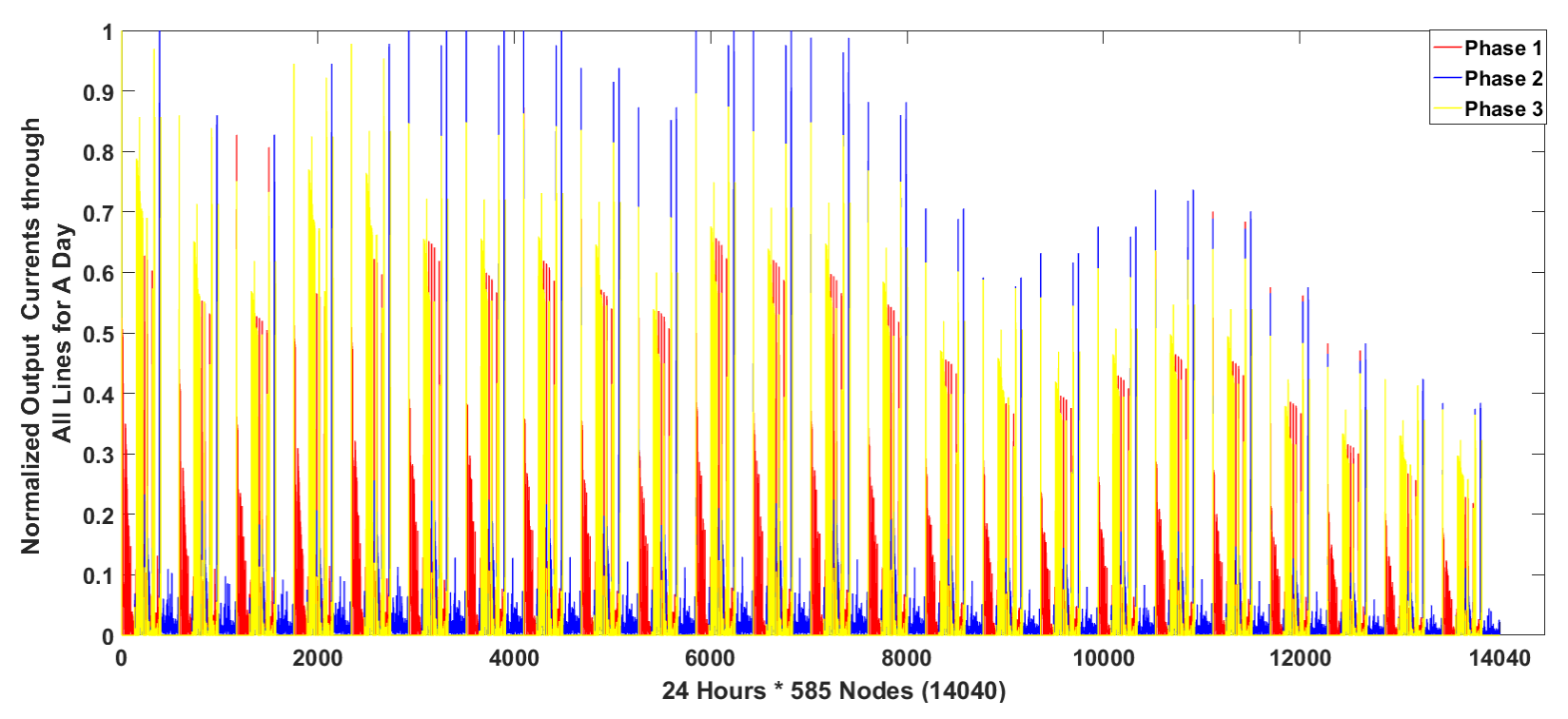

Fig. 4.14 Normalized Output Current

to create MLP model. TensorFlow is a user friendly opens source sequential application programming interface (API). It is a library which is used to create and perform many machine learning models and tasks. TensorFlow offers excellent functionality and high performance when working with large datasets [117].

3. Radial-basis function network: Similar to MLP, even for the RBFN model, size of the input vector is equal to 2809 . We have used only one hidden RBF layer. The number of RBF 


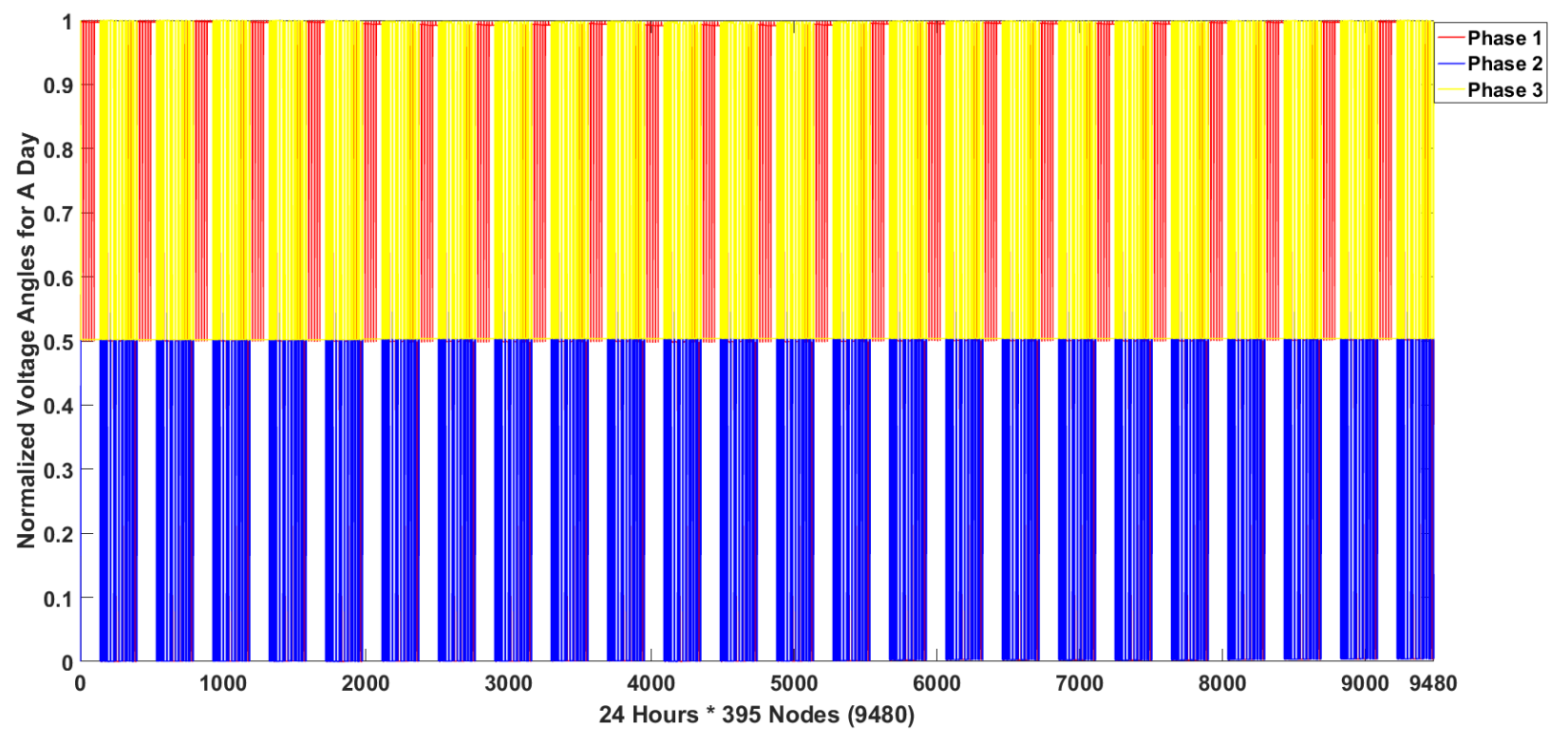

Fig. 4.15 Normalized Output Voltage Angle

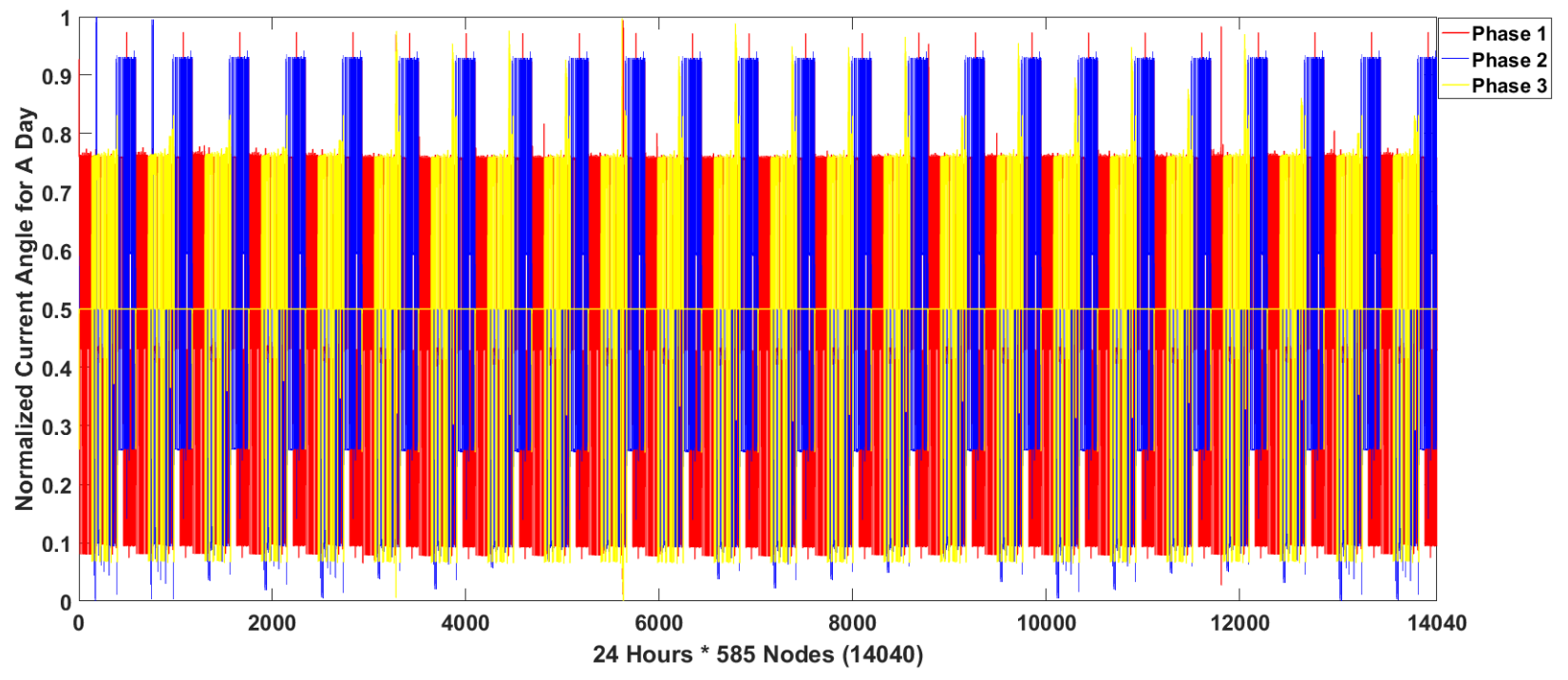

Fig. 4.16 Normalized Output Current Angle

neurons in the hidden layer is equal to 3500 . We have used K-means clustering for calculating the centers of the RBF neurons. We have minimized the mean square error loss for the output layer. The batch size was set to 1 with the learning rate equal to 0.0001 . We have trained the RBFN for 150 epochs.

Figures 4.13-4.16 represent the normalized output voltages, output currents, voltage angles, and current angles for a day for all three phases, respectively. 


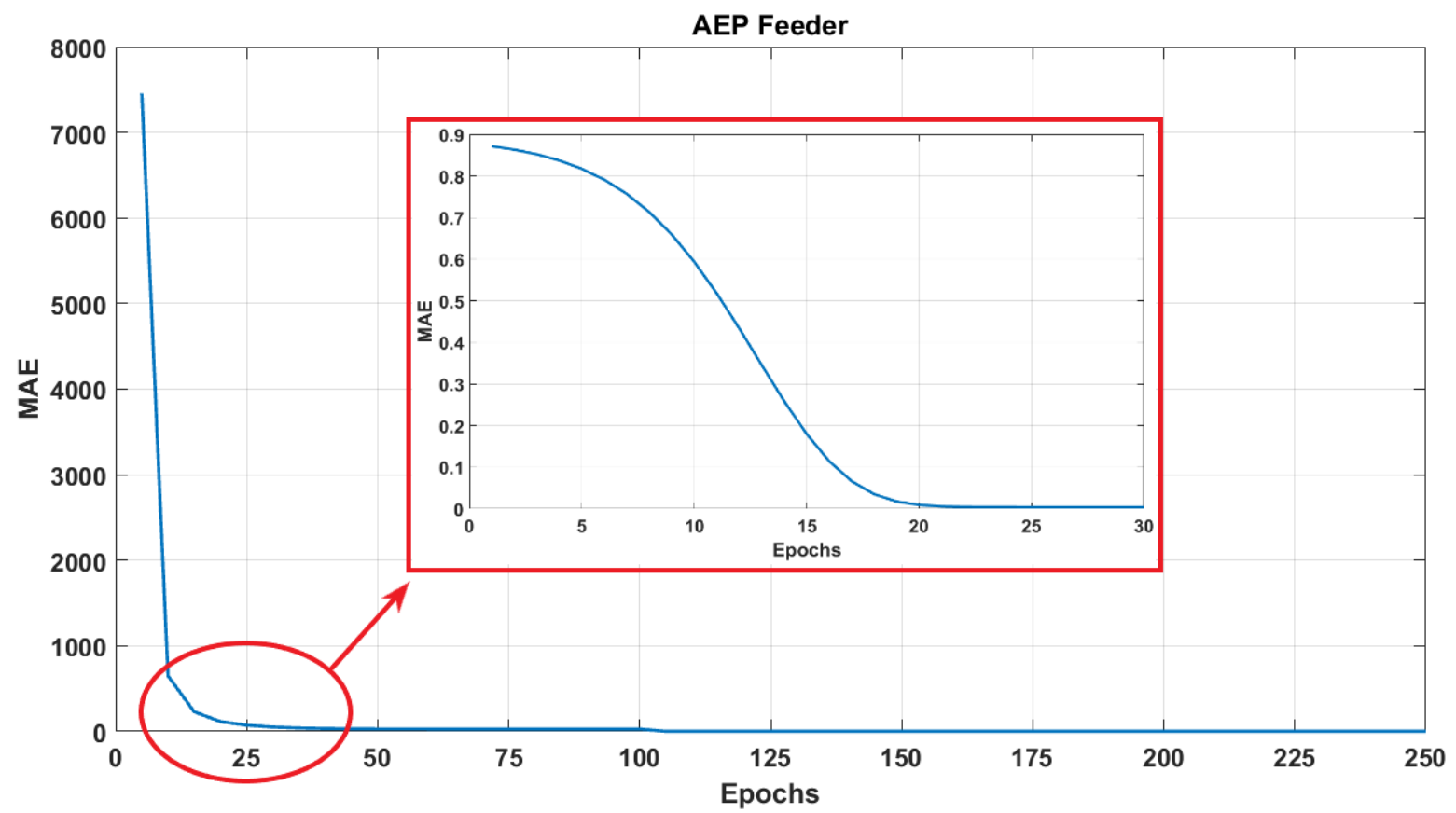

Fig. 4.17 Performance Evaluation- MAE

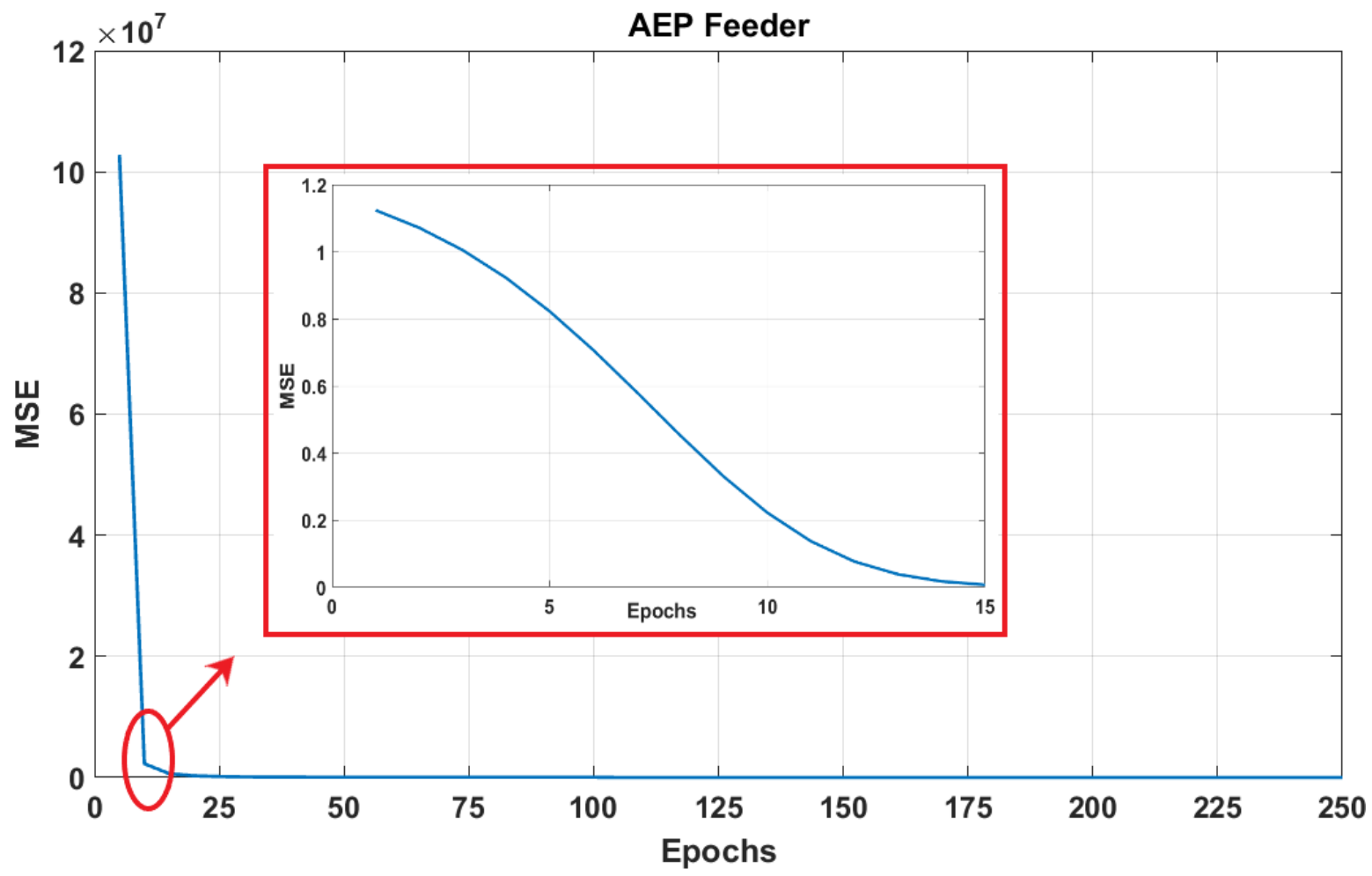

Fig. 4.18 Performance Evaluation- MSE 


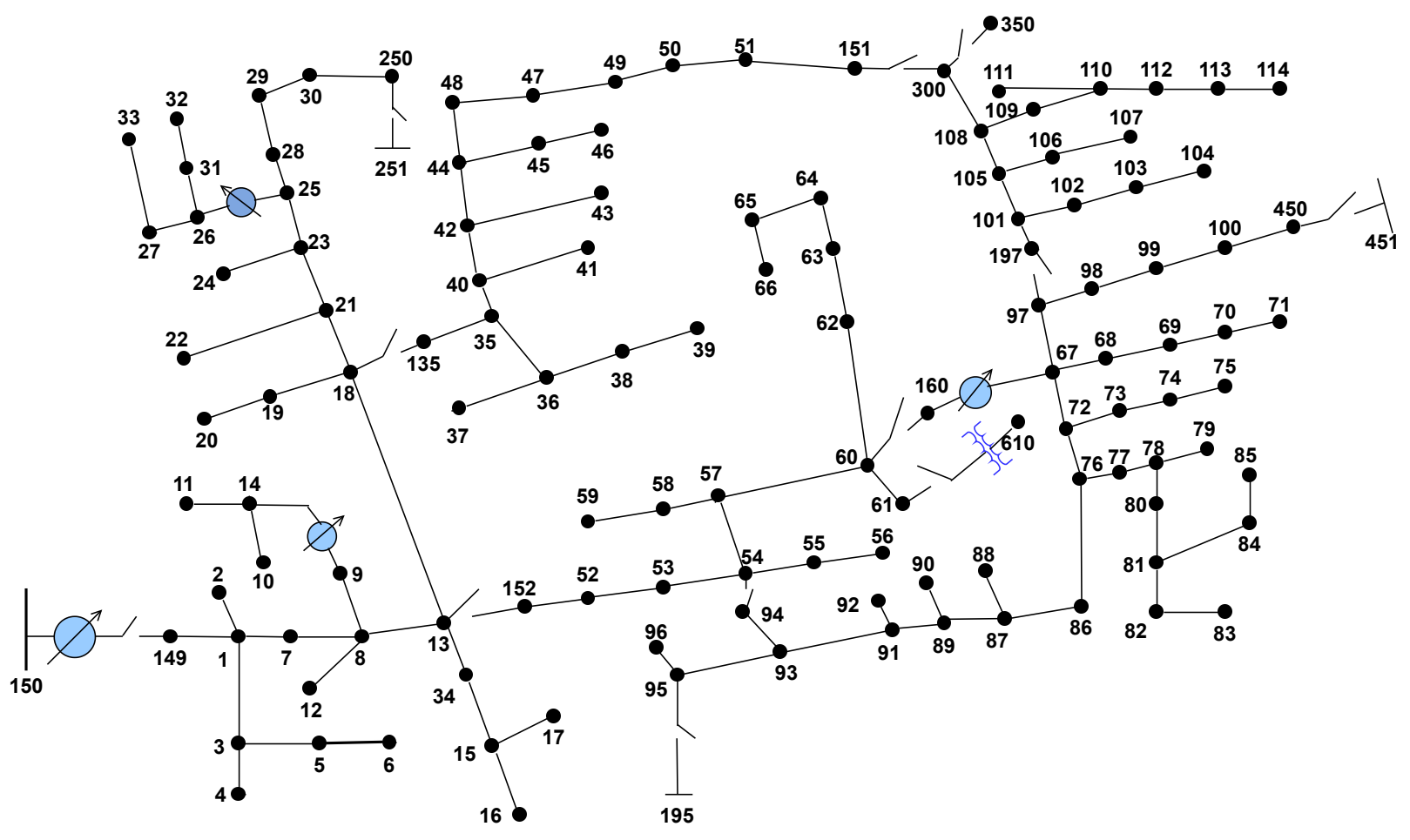

Fig. 4.19 IEEE 123 Bus Test Case Feeder

As discussed in section 4.4.2, we have used the min-max normalization to process this data and feed it to the neural networks. The accuracy of our proposed methodology is shown in figures 4.17 and 4.18 using the popular regression metrics like MAE and MSE. As discussed earlier, MAE and MSE show how well the NN has been able to predict the output or how closely it can predict for testing data. It is observed in figures 4.17 and 4.18 that both the metrics are going down to nearly zero after approximately 200 epochs. This implies that the AI model is able to predict the load flow quantities with very low errors.

\subsubsection{Case 3: IEEE 123 Bus Test Case}

To further test the feasibility of our proposed methodology, we have used IEEE 123 bus feeder [118], shown in figure 4.19. In this standard IEEE test feeder, we have 92 load buses, consist of single and three phase balanced and unbalanced loads. The load-shapes are generated from actual load data of New York state [119]. We have generated the power flow inputs for 1 year using the OpenDSS MATLAB COM platform.

So for the total 8760 data points generated, we have selected 6000 points randomly as the training dataset while remaining 2760 data points used for testing the AI model. Considering the AEP distribution network, the input vector dimension for the $\mathrm{NN}$ is equal to 2809 and the number of classes or the outputs is equal to 5880 . 


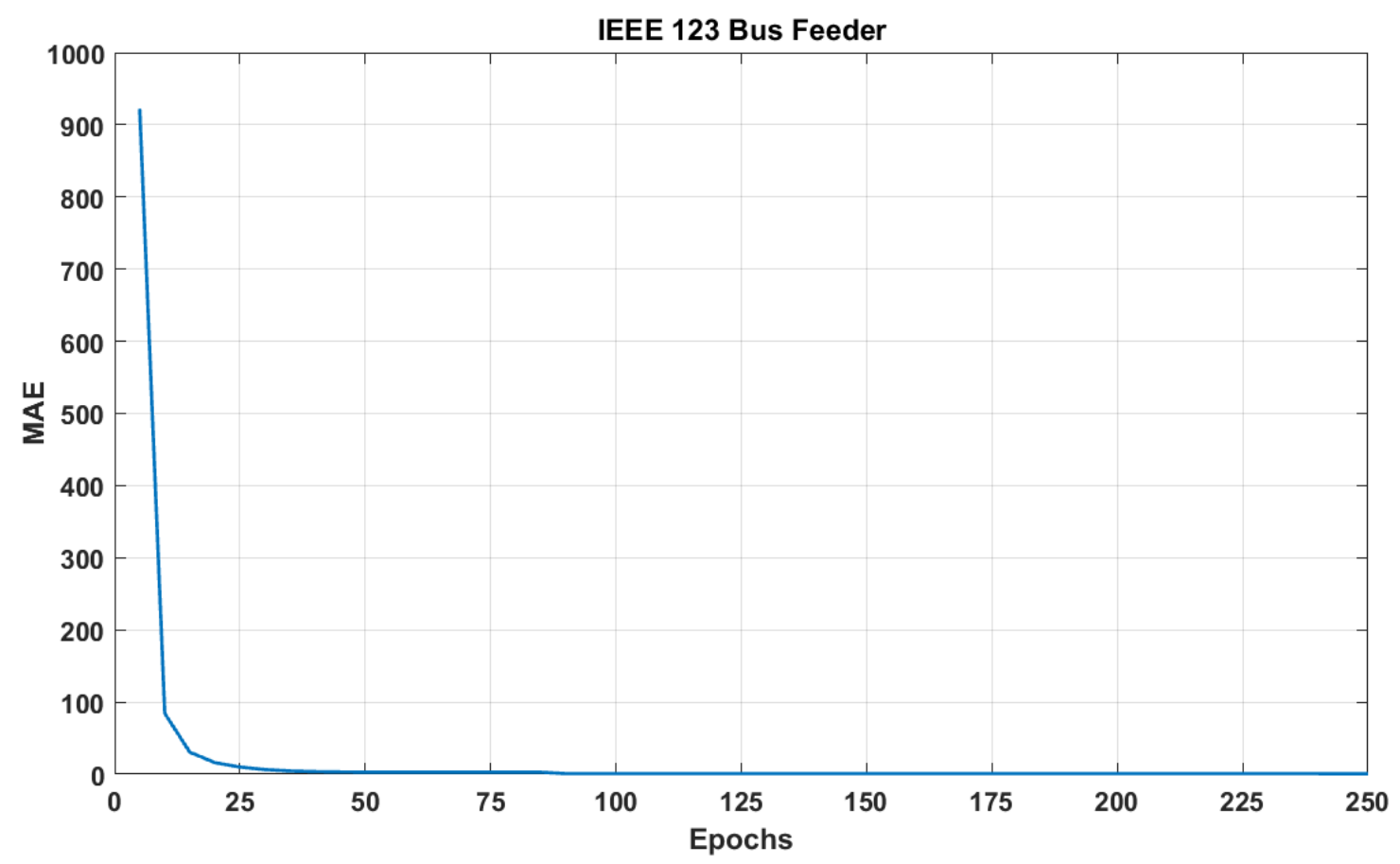

Fig. 4.20 Performance Evaluation- MAE

Similar to the AEP feeder test case, we have used three NN models in this case as well:

1. Convolutional neural network: The input vector has For the CNN model training and testing, the input vector has 1521 columns constructed from the input information of the feeder like voltage, voltage angles, loads, and line parameters, while there are 8760 rows where each row represents an hour from the quasi-static time series simulation of one year. This 1521 is reshaped into $39 \times 39$ to make it a square which is the acceptable format for input to $\mathrm{CNN}$.

2. Multi-layer perceptron : for this network, the input vector size remains 1521 while the output vector has 2172 data points for 8760 hours. For the MLP model, we have used five hidden fully connected layers of size 2048, 1536, 1024, and 512 in that order. Adam optimizer with momentum as 0.2. To check the system on different learning rate, it is fixed at 0.009 .

3. Radial-basis function network: The size of input and output vectors remain same in three cases. Unlike AEP system, the number of RBF neurons in the hidden layer is reduced to 2500. The batch size was set to 1 with the learning rate equal to 0.0001 .

Figures 4.20 and 4.21 are the plots for MAE and MSE vs number of Epochs for this test case. As it is evident from the figures that for the initial epochs, MAE and MSE start off with a huge error but after a few epochs, error is close to zero. This validates our claim that AI models are 


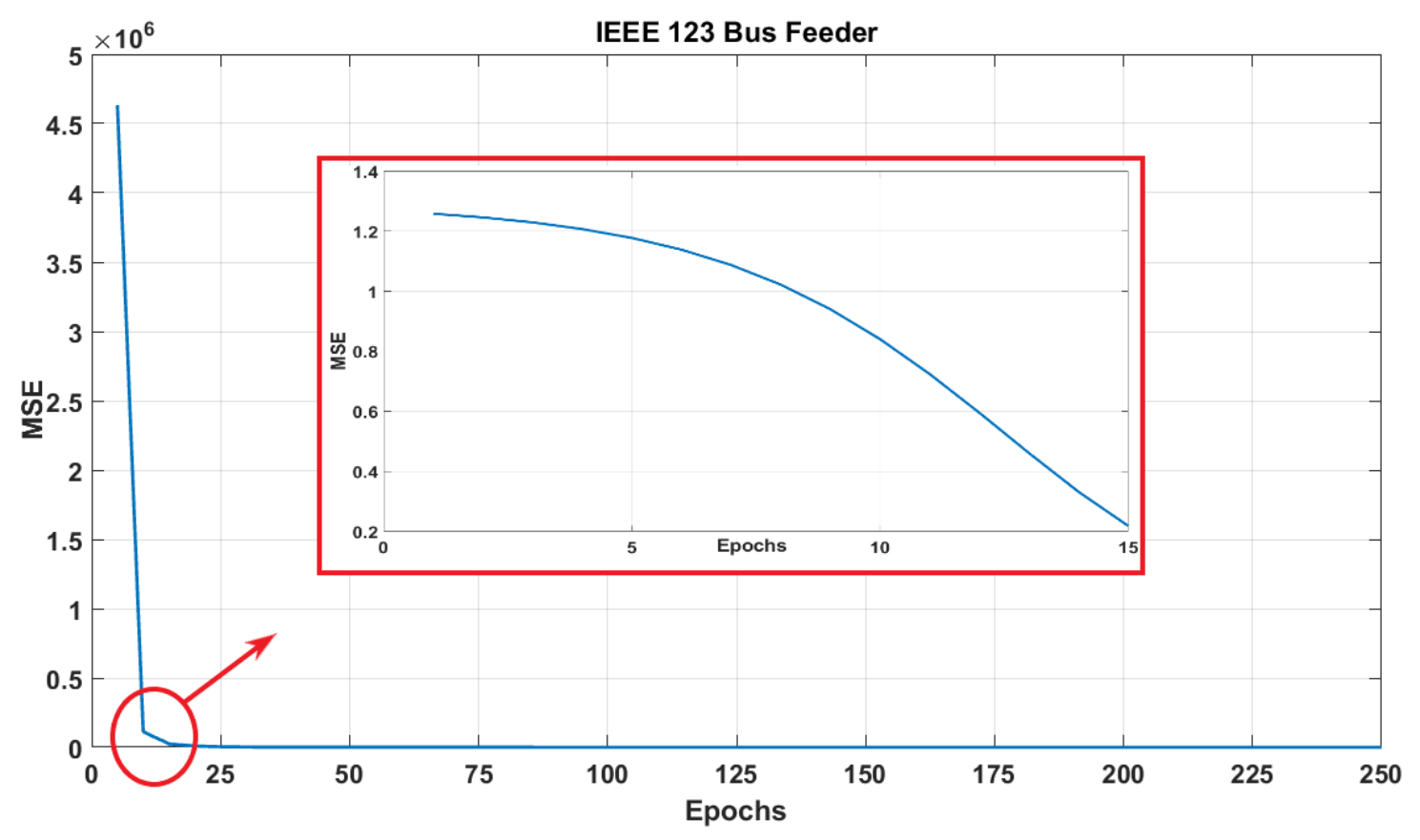

Fig. 4.21 Performance Evaluation- MSE

Table 4.2 Performance Comparison Results

\begin{tabular}{|c|c|c|c|c|c|c|c|}
\hline Test Cases & Scenarios & \multicolumn{2}{|c|}{ RBFnet } & \multicolumn{2}{c|}{ MLP } & \multicolumn{2}{c|}{ CNN } \\
\hline & & MSE & MAE & MSE & MAE & MSE & MAE \\
\hline \multirow{3}{*}{ IEEE 4 Bus } & Constant PQ Load & $0.12 \%$ & $0.33 \%$ & $0.08 \%$ & $0.36 \%$ & $0.07 \%$ & $0.35 \%$ \\
\cline { 2 - 8 } & Constant Impedance Load & $0.11 \%$ & $0.33 \%$ & $0.08 \%$ & $0.36 \%$ & $0.06 \%$ & $0.35 \%$ \\
\cline { 2 - 8 } & ZIP Load & $0.12 \%$ & $0.32 \%$ & $0.19 \%$ & $0.95 \%$ & $0.06 \%$ & $0.36 \%$ \\
\hline AEP Feeder & Constant PQ Load & $0.11 \%$ & $0.34 \%$ & $0.042 \%$ & $0.43 \%$ & $0.04 \%$ & $0.42 \%$ \\
\hline IEEE 123 Bus & Constant PQ Load & $0.70 \%$ & $0.50 \%$ & $0.85 \%$ & $0.47 \%$ & $0.60 \%$ & $0.10 \%$ \\
\hline
\end{tabular}

predicting the load flow quantities with nearly zero error. Table 4.2 is the comparison of results for all the test cases. For IEEE 4 node, loads were modeled for three scenarios like constant PQ load, constant impedance and ZIP load. The prediction accuracy metric MAE and MSE are found to be very low for all the networks. In almost all the test cases, $\mathrm{CNN}$ has shown to be very accurate for predicting the output variables. This table represents the performance result for three feeders for base cases with excluding any severe events or stochastic behaviour in the grid. However, in the next section 4.8 , we present some scenarios which result in bringing uncertainty to the line flows. 


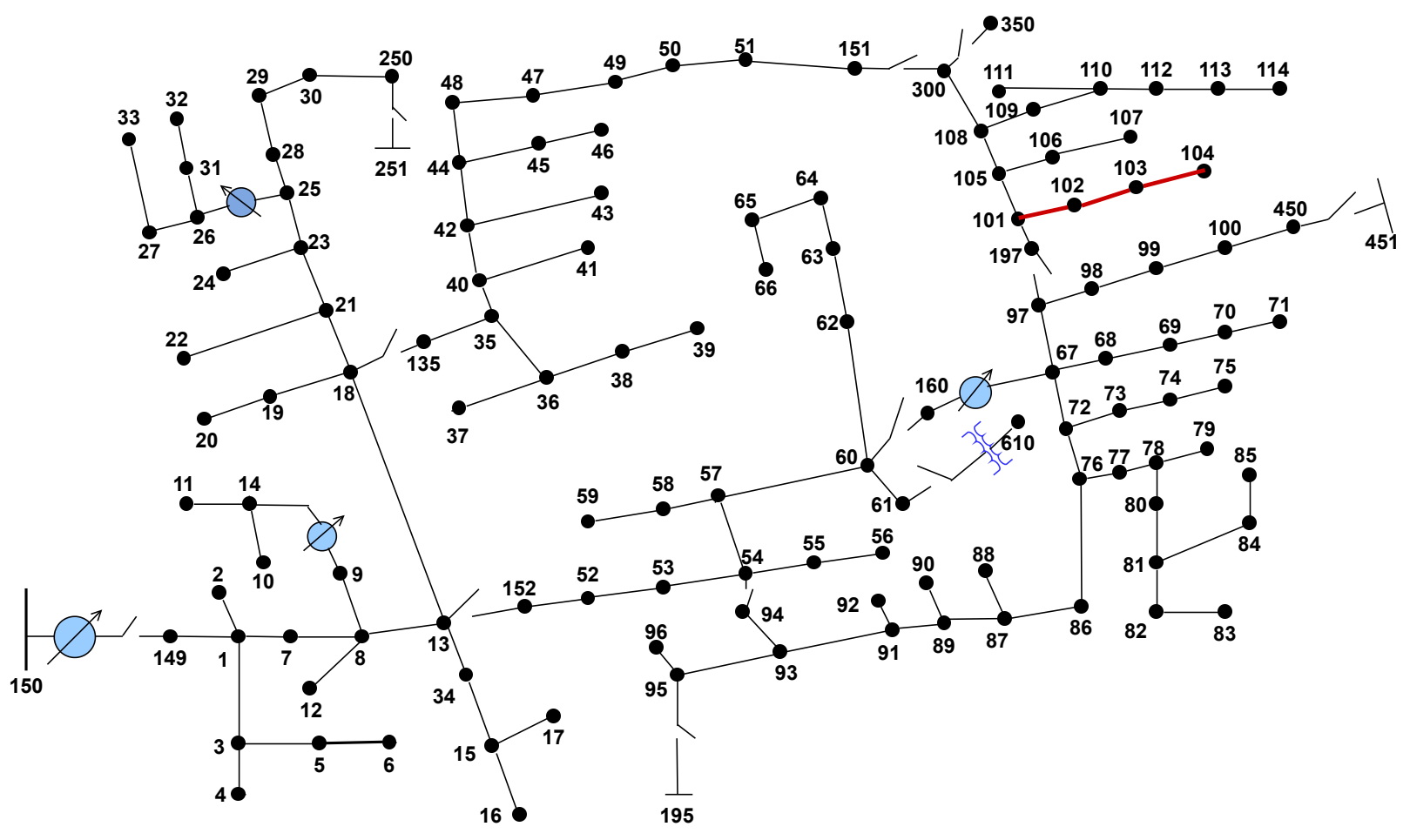

Fig. 4.22 Circuit plot when topology changes

\subsection{Case Studies}

In this section, we are modifying the IEEE 123 bus feeder to accommodate different scenarios and predict power flow efficiently.

\subsubsection{Topology Change}

Power system is a complex network of various components like generators, substations, transmission lines, distribution transformers, and loads. Topology of a power system is defined by connectivity of these components. To better estimate the power flow during events like hurricanes, tornadoes, which affects the power lines and substations, and can result in blackouts, we perform the $\mathrm{N}-1$ contingency analysis. Contingency analysis is one of the most important tasks encountered by the planning and operation engineers of bulk power system. Its purpose is to analyze the power system in order to identify the overloads and problems that can occur due to a "contingency". If a system is $\mathrm{N}-1$ contingent it means that the system can continue to operate within nominal limits if 1 element fails. We modified the topology of the feeder by removing 3 lines and generated the data for our AI model which is used to predict the power flow in the three phase unbalanced distribution power system. To train and test the data, AI model needs some modification as well. 


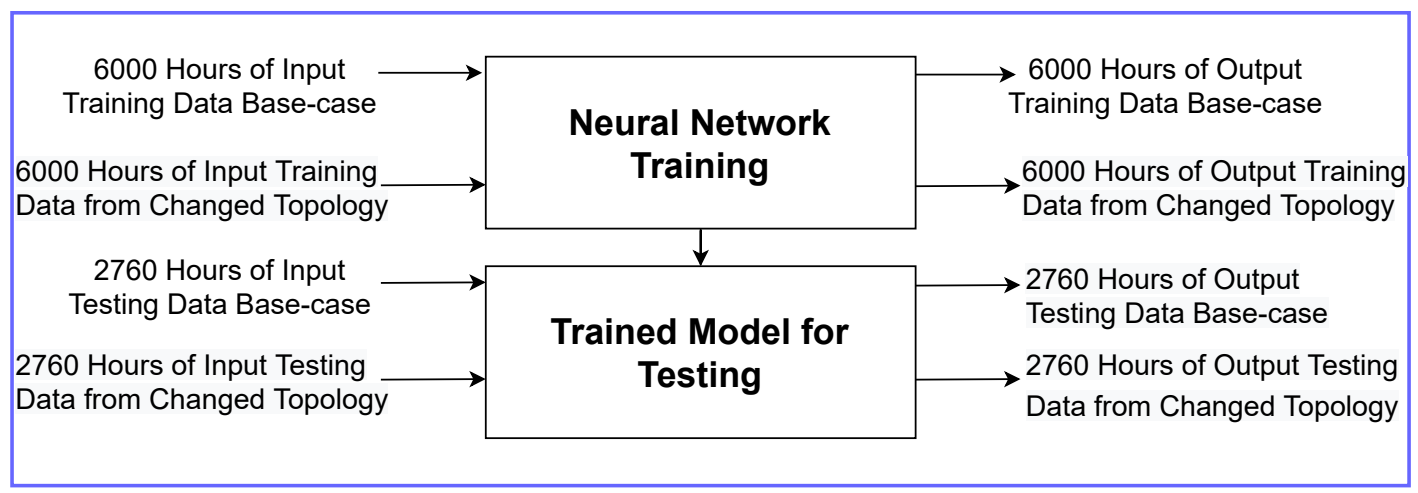

Fig. 4.23 Mixing Training and Testing Data

\section{Example:}

To show the impacts of topology change, we have modeled the feeder in OpenDSS software. In figures 4.19 and 4.22, we have shown the comparison of circuit plots when the topology of the feeder changes. The circled line is removed as part of N-3 topology change which results in two other line segments (pink line) removed from the network. The input data has voltages, line parameters, and currents as zero for the removed lines, that brings the variability in the test data. Our objective is to change the topology of the system and predict the power flow using the deep neural networks. To demonstrate the feasibility of our proposed method, we have used IEEE 123 bus test feeder. To train the AI network, we used the dataset generated from section 4.7.3, while testing is performed using the newly generated data from the feeder after creating the topology change. For topology change scenario, Load flow prediction is performed using $\mathrm{CNN}$ which is found to be more robust and efficient.

\section{Results for Topology Change:}

We used two methodologies to show the results for topology change and measure the effectiveness of our proposed models.

- Mixing the data: In this method, we trained and tested generated before and after topology change.

As shown in figure 4.23, to train the model we used the 6000 hours of input and data from base case and changed topology scenarios, while testing is performed using 2760 hours of data. With this method, the model gets to see the topology change data before testing. Figure 4.24 shows the performance plot for this method. For initial epochs, the MAE error is very high but as the epochs increases, MAE continues to go close to zero as shown in the subplot.

- Generalization using Dropout: Deep neural networks are at risk to overfit a training dataset. Overfitting refers to the phenomenon where models learn the data too well or sort of memo- 


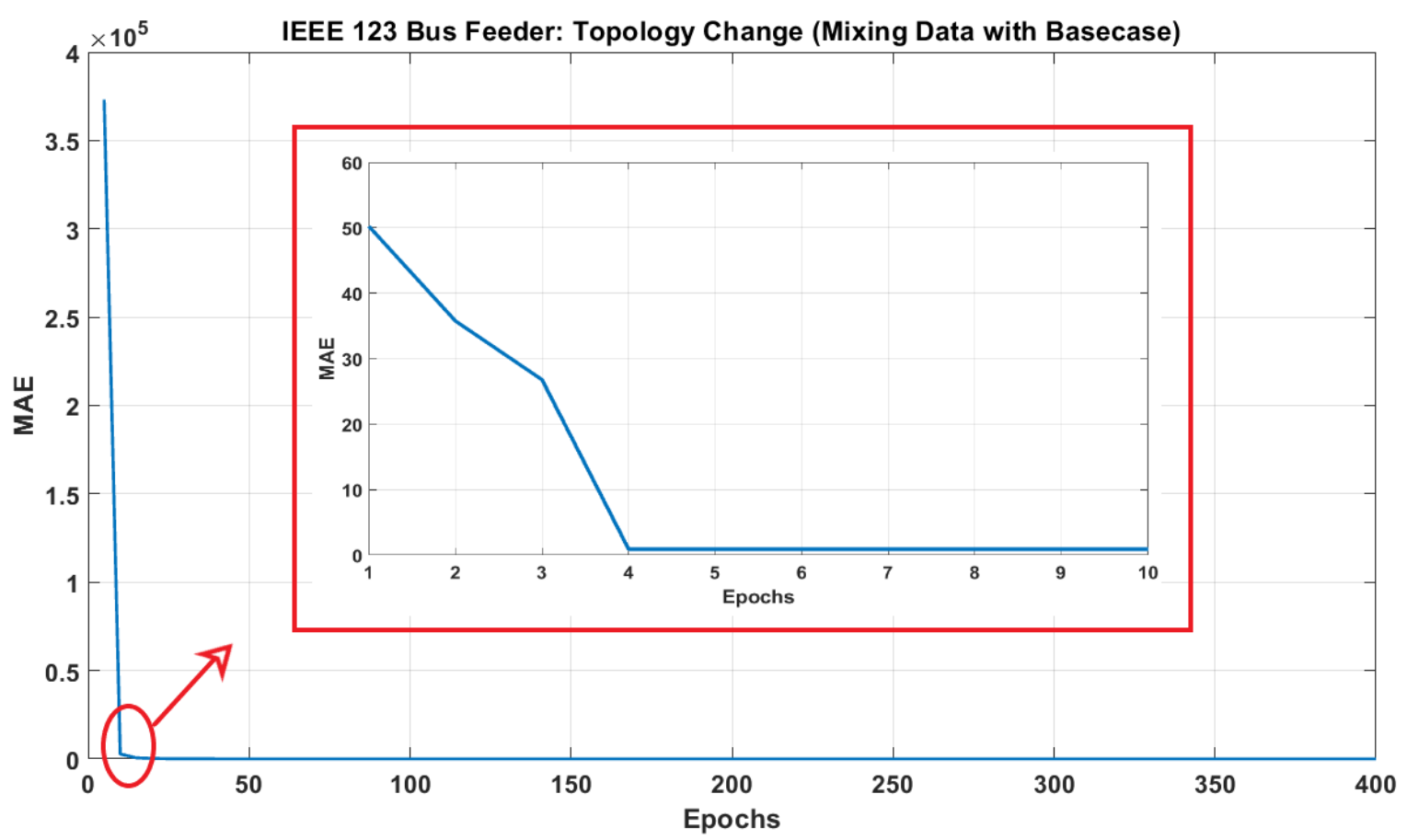

Fig. 4.24 MAE- Mixing the Training Data

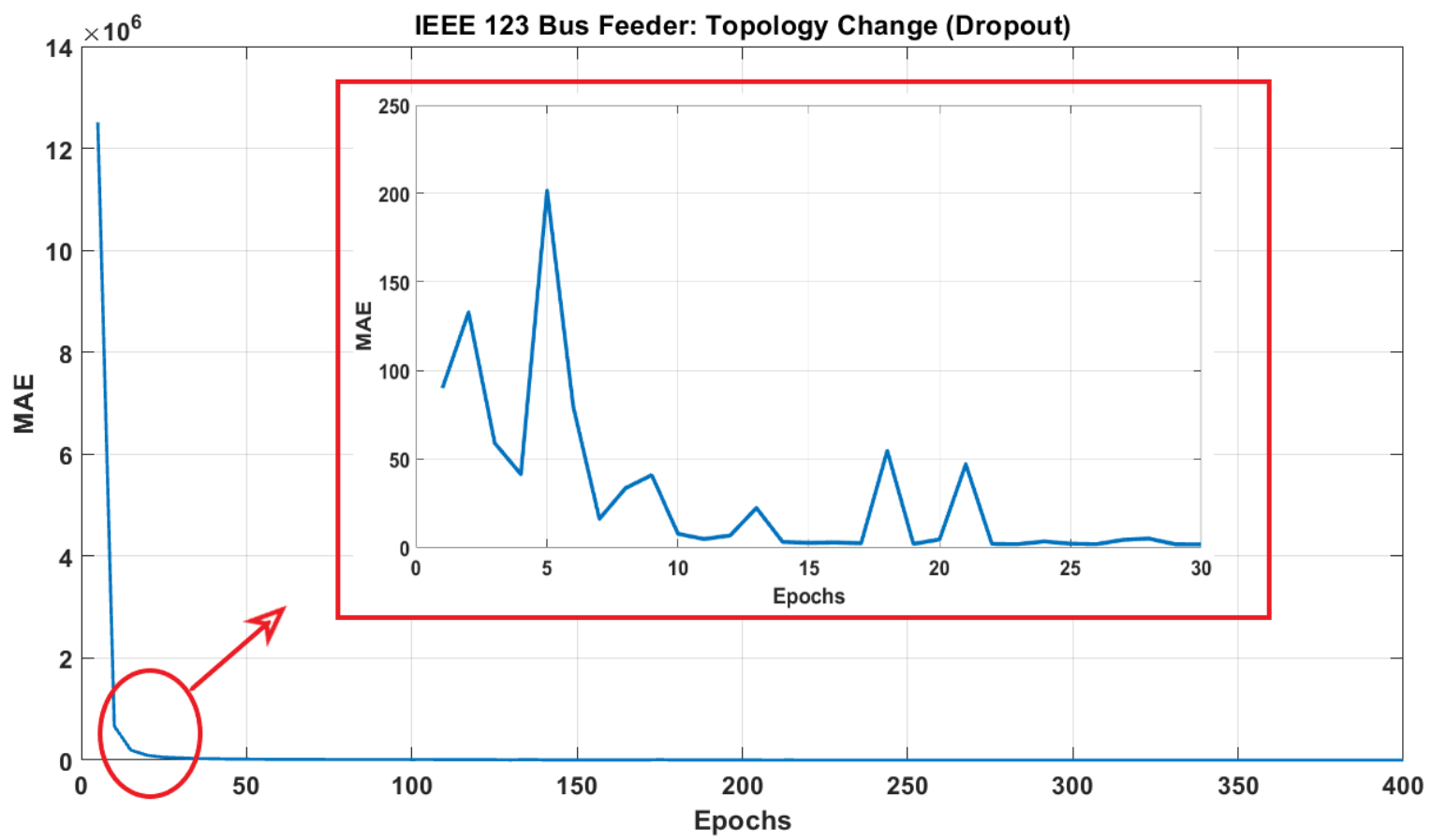

Fig. 4.25 MAE- Dropout

rizes the training data which results in poor performance when model is evaluated on testing data. 


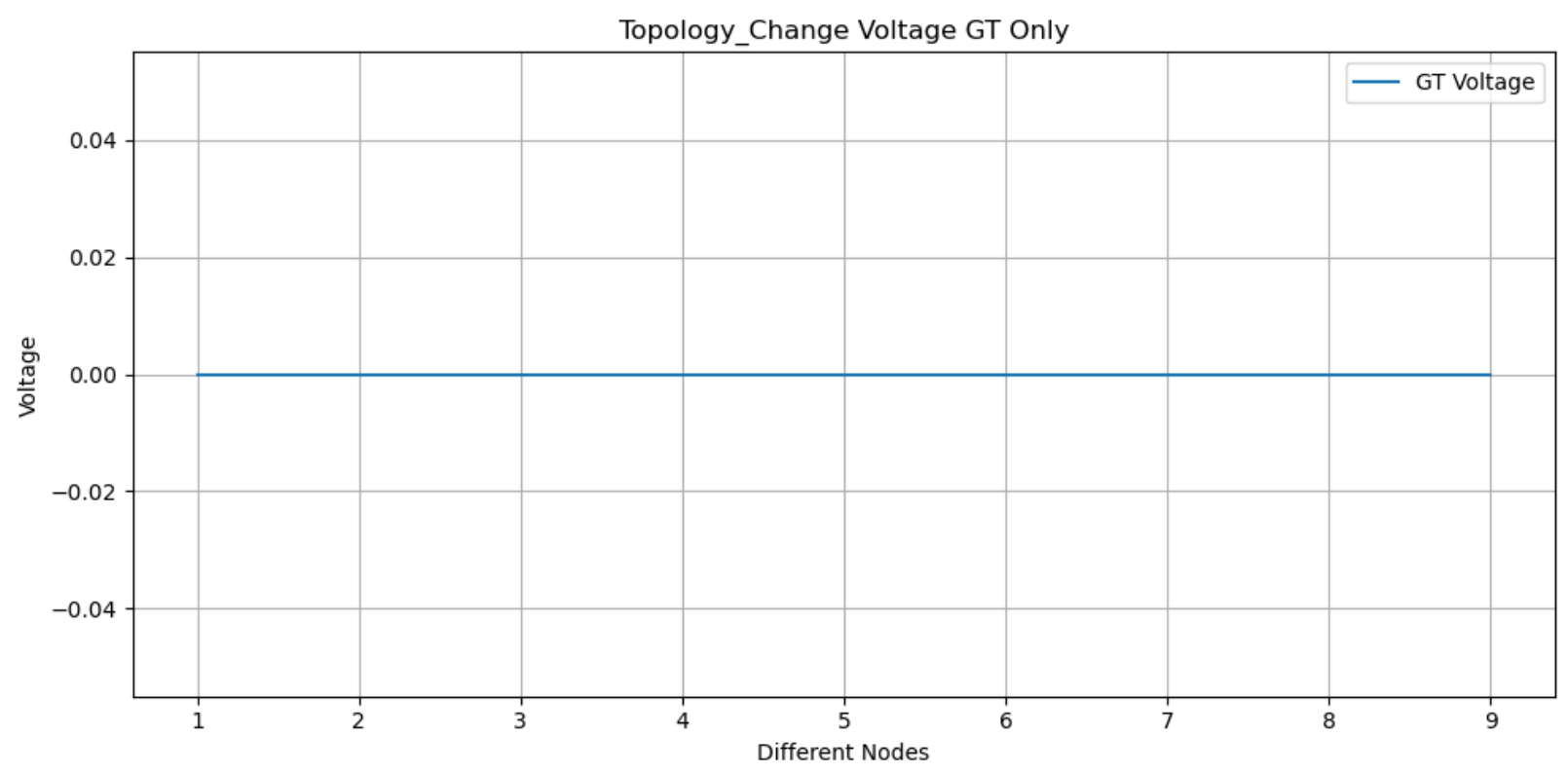

Fig. 4.26 Voltage for OpenDSS(GT)

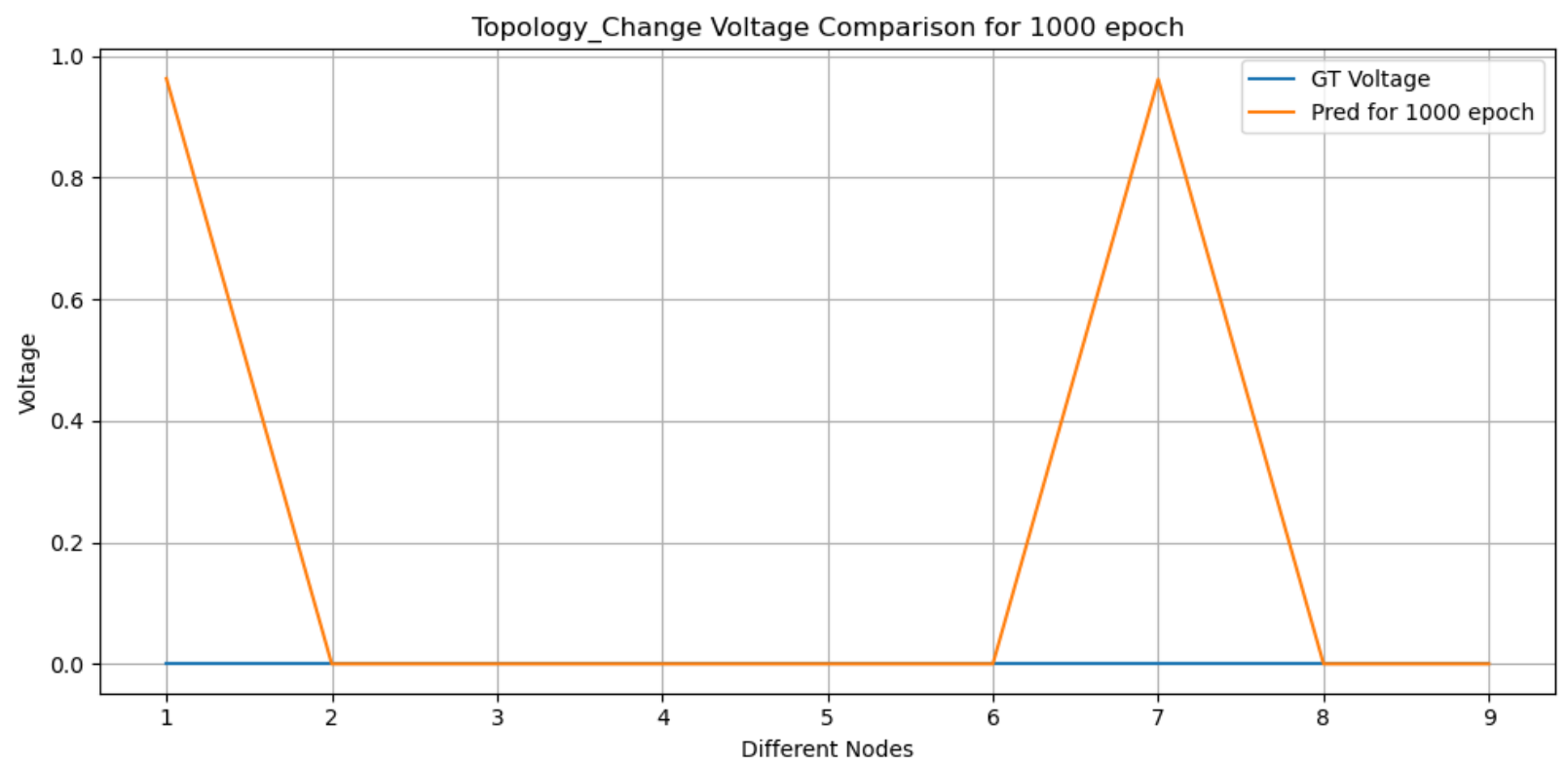

Fig. 4.27 Voltage for OpenDSS (GT) vs AI

To overcome overfitting, generalization is used. Here, we have used the dropouts for generalization where some layer outputs are randomly disconnected or dropped from the training process.

Figure 4.25 shows the accuracy result when dropout is considered. Figures 4.26 and 4.27 show the comparison between the AI prediction values and ground truth (OpenDSS output). As we have 


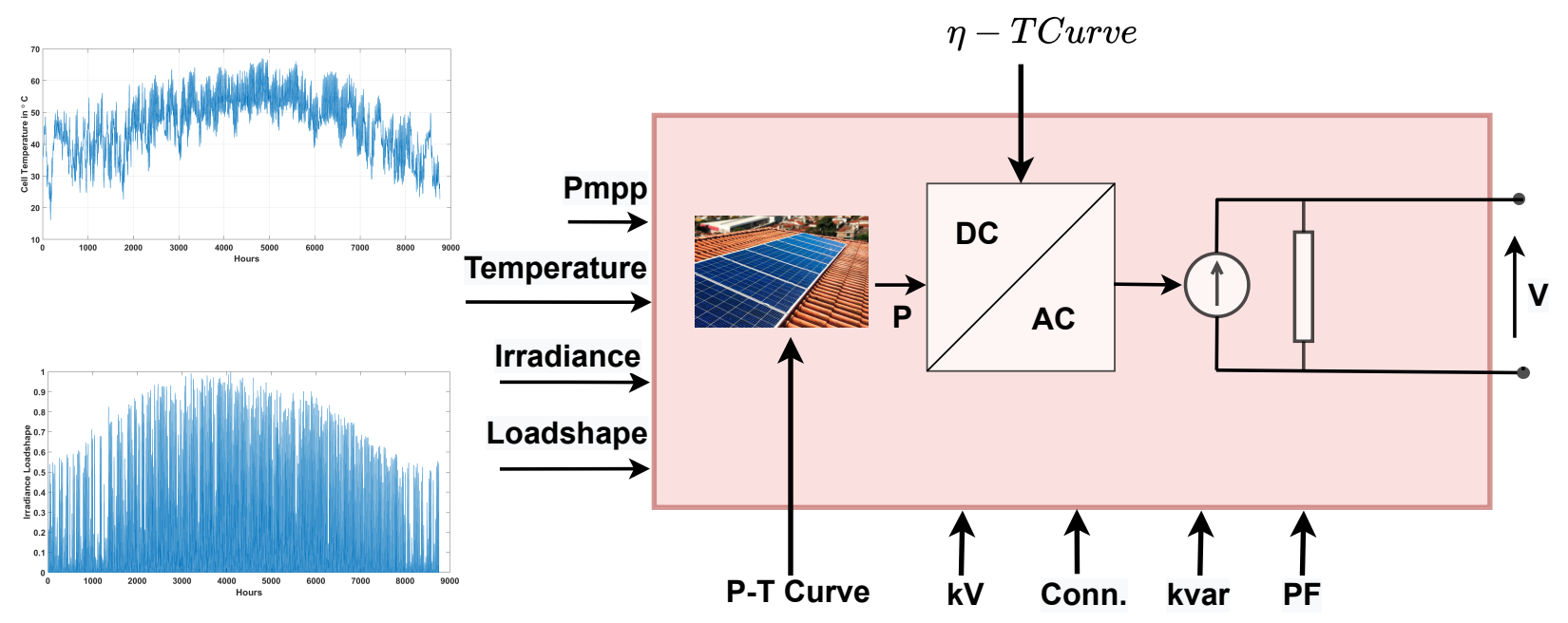

Fig. 4.28 Block Diagram of the PV System Model

removed 3 lines to show the topology change, the voltage values are zeroes for those lines in each phase. From figure 4.27, it is evident that our AI model is able to predict seven such zero points while two points are not predicted accurately. However, the model did not face such non linearity in data while training. For unseen data which is generated from topology change, our AI model is still very accurate and robust as the overall error is very low.

\subsubsection{PVs Integration Impact on Power Flow}

It is estimated that by 2050, the renewable energy generation could contribute to around $80-90 \%$ of annual electricity, including 49-55\% from wind and solar photovoltaic generation [120]. Such increased penetration of Distributed generation (DG) may affect the distribution feeder voltage regulation. As more renewables are integrated into the grid, their intermittent nature can pose problems for grid operators in terms of forecasting and meeting load. We proposed to predict the power flow in a feeder which has multiple rooftop PVs $(5-500 \mathrm{~kW}$ residential solar panels) using the deep neural network.

\section{Example:}

Figure 4.33 shows the IEEE 123 bus feeder which has many EV charging stations and rooftop solar in the grid. In our work, we have considered many locations throughout the feeder with these distributed energy resources.

PV Modeling: Figure 4.28 provides the detailed PV model information. The parameters needed to model the PV in OpenDSS are: Temperature and temperature factor, efficiency of inverter, Power, Irradiation curve (Loadshape), Temperature curve, Temperature coefficient (Pmpp).

Get/set the rated max power of the PV array for $1.0 \mathrm{~kW} / \mathrm{sq}-\mathrm{m}$ irradiance and a user-selected 


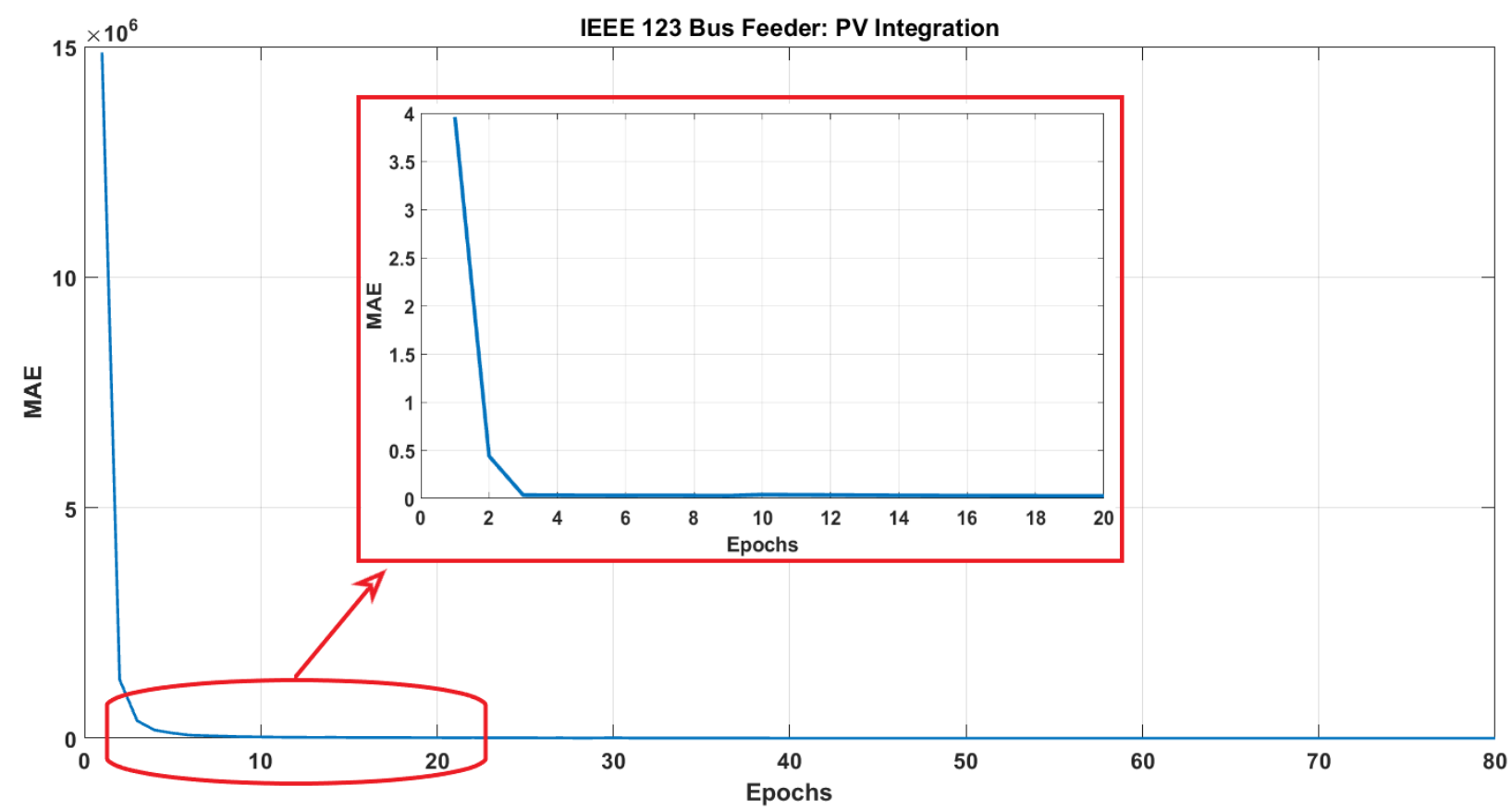

Fig. 4.29 MAE- PV Integration

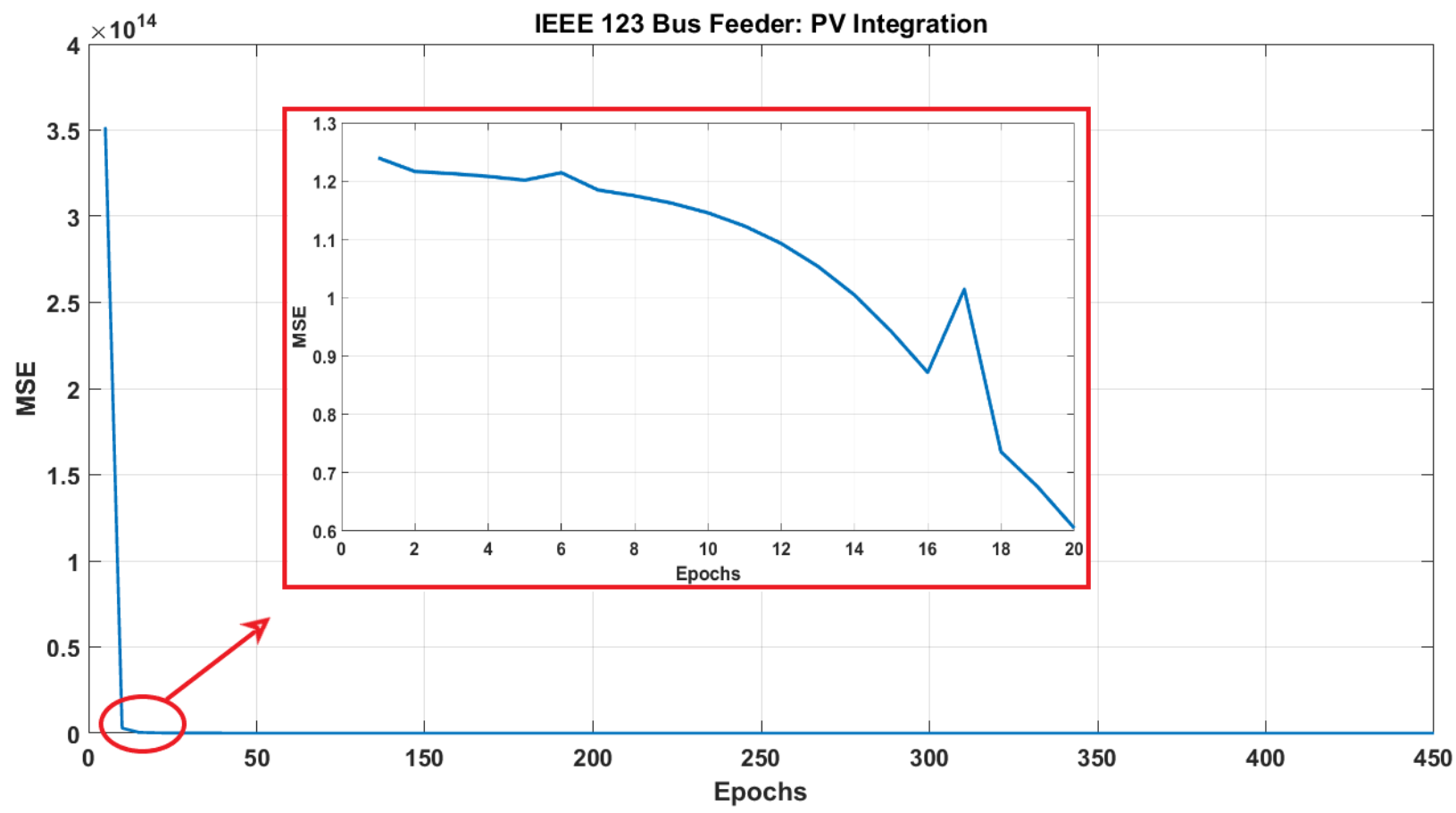

Fig. 4.30 MSE- PV Integration

array temperature for the calculation of PMPP. The P-T Curve should be defined relative to the selected array temperature. The irradiance and air temperature data are used from [121].

We have used the convolutionary neural network to predict the power flow in case of penetration 


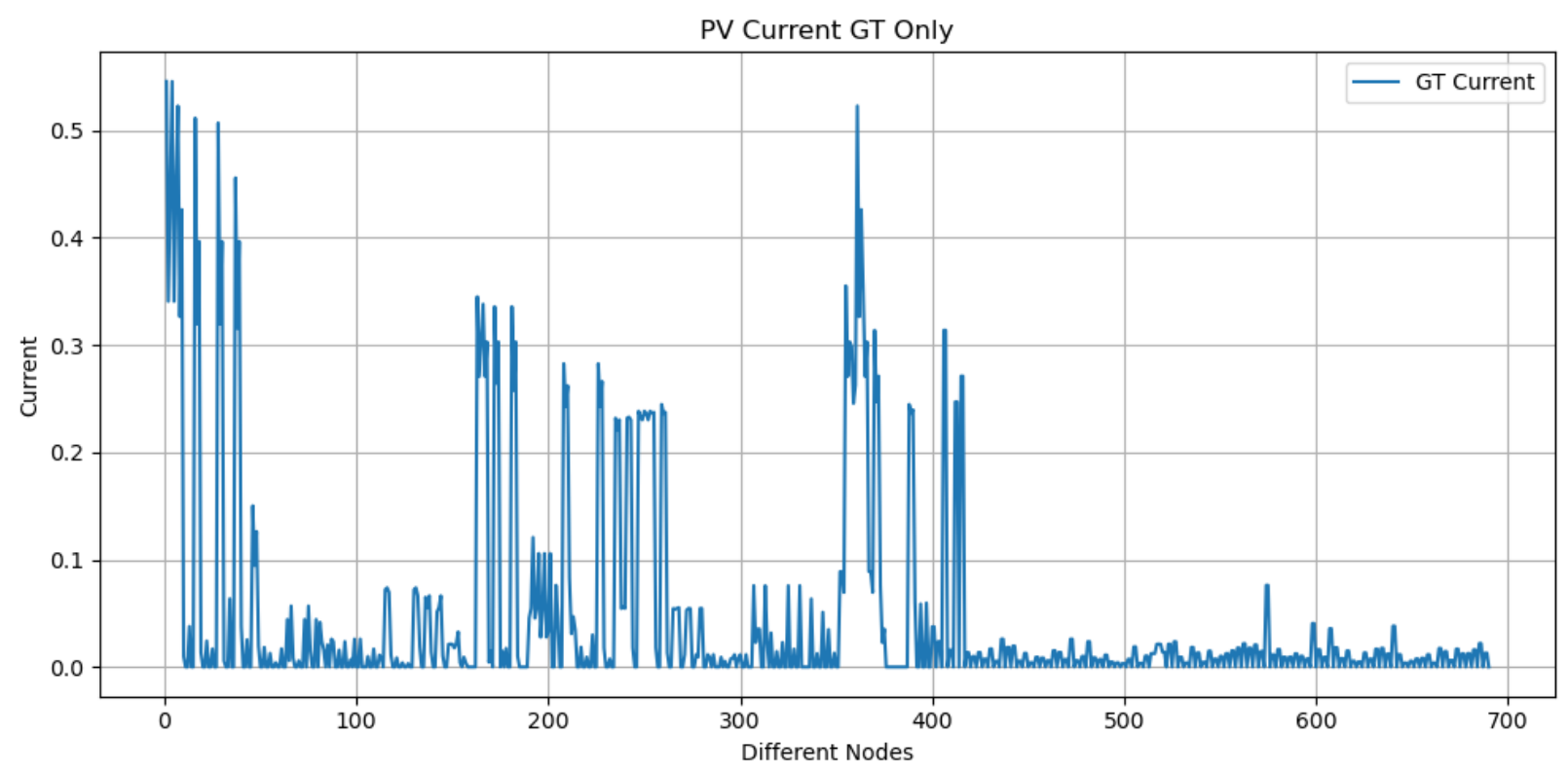

Fig. 4.31 OpenDSS(GT) Currents

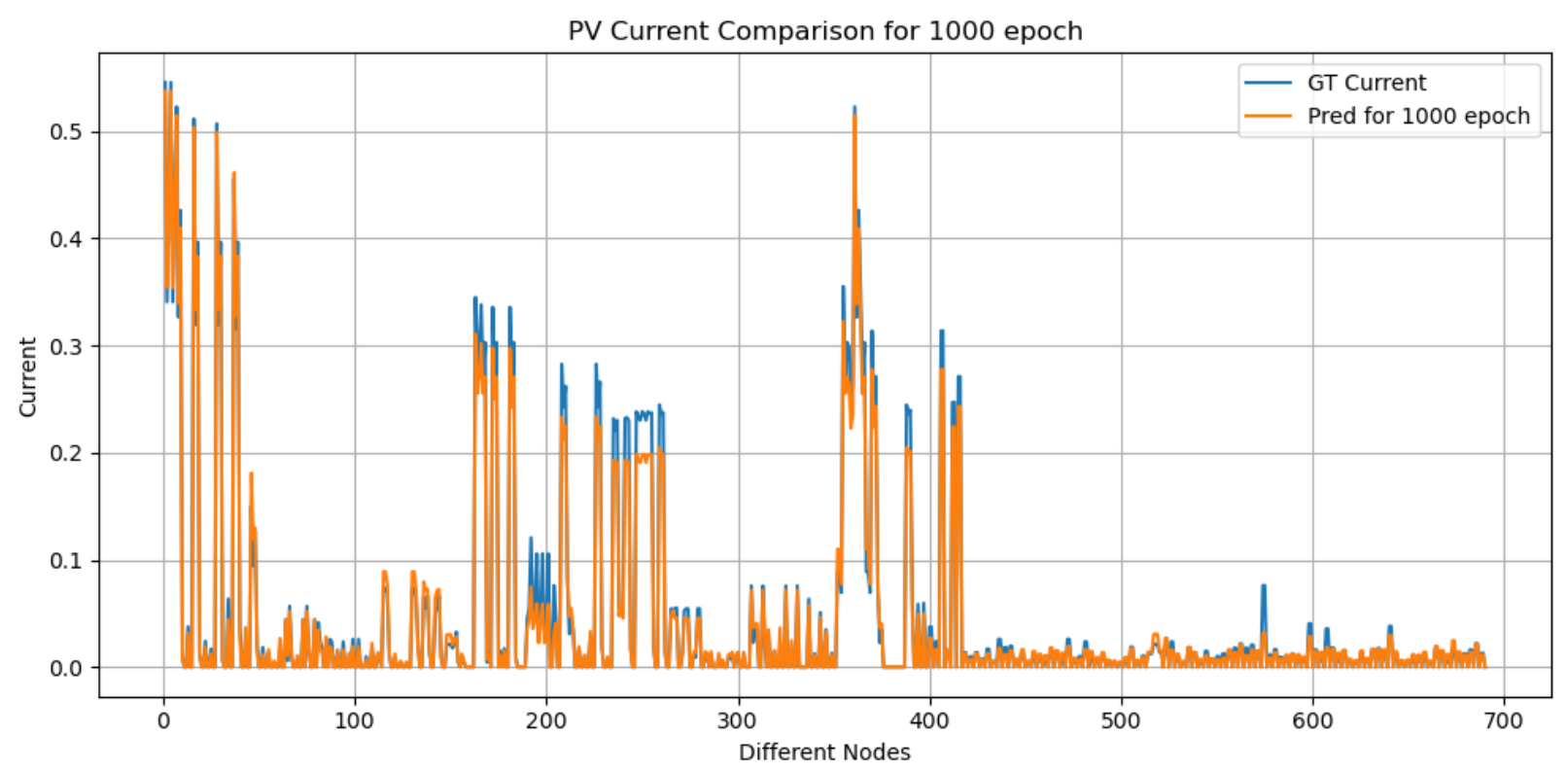

Fig. 4.32 Currents for OpenDSS(GT) vs AI

of PVs into the grid. Figures 4.29 and 4.30 show the performance accuracy when PVs are added to the grid. The testing and training data are generated considering multiple rooftop PVs throughout the feeder. Figure 4.31 plots the currents in p.u. which is mainly the ground truth values taken from OpenDSS output. As it is evident from figure 4.32, the non linearity due to PV integration is predicted. However, the in some instances, our model is not able to predict very accurately. 


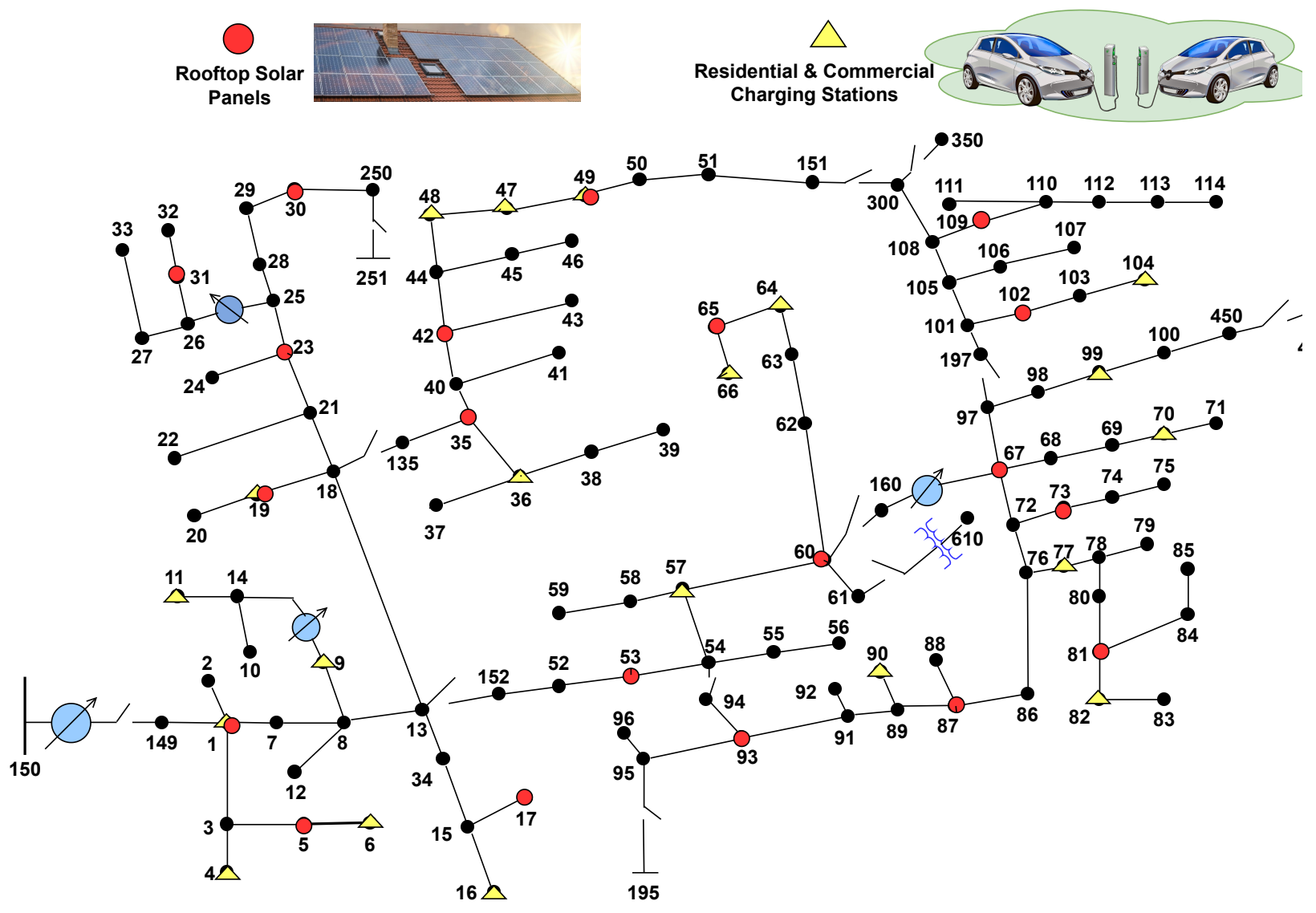

Fig. 4.33 IEEE 123 Bus Feeder with Renewable Energy Sources

\subsubsection{EVs Integration Impact on Power Flow}

As shown in figure 4.33, we have considered residential EVs charging station at many different locations throughout the feeder. Due to the random driving and simultaneous charging behaviour of EV owners can overload the grid during the peak hours. So it is vital to analyze the adverse impacts of such EV penetration into the grid. Electric vehicles can be modeled in OpenDSS as a load for grid to vehicle mode while for vehicle to grid mode in which vehicle can supply energy back to grid, EV can be modeled as battery or negative loads. The optimized charging profiles from [122] is used to model the EVs. These profiles are used for quasi static time series simulation. We used OpenDSS- MATLAB COM interface to generate the load flow data for one year in a AEP utility feeder in the presence of EVs.

Table 4.3 shows the error in percentage for all the three scenarios for the CNN model for modified IEEE 123 bus feeder. For each case, the model is trained using the basecase IEEE 123 bus feeder while testing data is generated by each scenario so the model is fed through a variation in data. 
Table 4.3 Prediction Error

\begin{tabular}{|c|c|c|c|}
\hline Test Cases & Scenarios & \multicolumn{2}{|c|}{ CNN } \\
\hline & & MAE & MSE \\
\hline \multirow{3}{*}{ IEEE 123 Bus } & Topology Change & $2.40 \%$ & $1.30 \%$ \\
\cline { 2 - 4 } & PV Integration & $1.60 \%$ & $1.40 \%$ \\
\cline { 2 - 4 } & EV Integration & $1.50 \%$ & $0.90 \%$ \\
\hline
\end{tabular}

\subsection{Conclusion}

In this work, we are performing the load flow prediction using deep learning approaches for three phase unbalanced distribution network. As utilities do not share the exact network due to security reason, we aim to replace their feeder model using our trained neural networks which predicts the power flow with very low error for different feeders. With our proposed method, we are also aiming to get rid of conventional iterative based power flow methods which are time consuming. Our trained neural network models are able to predict the load flow quantities with very low errors. The training data was collected from OpenDSS MATLAB COM interface for all test cases. We proposed our methodology using RBF network, MLP, and CNN. We also considered different scenarios like topology changes in the feeder, renewable energy sources penetration into the grid and found the prediction error to be very low in all scenarios. 


\section{CHAPTER 5}

\section{Conclusion and Proposed Future Work}

In this research work, we have presented artificial intelligence application to predict power flow in the three phase distribution network. Main contributions of the dissertation is mentioned as follows:

\subsection{Conclusion}

- In chapter 2, we discuss some of the problems related to the uncontrolled charging and its adverse effects on the grid. First, we minimize the load variance in distribution system by leveraging the charging coordination of aggregated EVs. Minimizing the load variance ultimately maximizes the load factor of the distribution grid and minimize the power losses. This can also be called as peak load shaving or load levelling techniques. In the next part, a social welfare problem is formulated as a primal dual convex optimization problem for optimized EV charging which maximizes the profits of vehicle owners as well as the grid. To check the feasibility of the proposed methods, we performed the load flow on the IEEE 13 bus and 34 bus test feeders to observe any constraints violations. In the last problem in this chapter, we find the optimal scheduling for Distributed energy resources including battery energy storage system and for EV charging and discharging schedule. A linear programming is used to solve this cost minimization problem.

- Chapter 3 introduces the concept of the Distributed Resource Allocation(DRA) approach for incorporating a large number of Plug-in EV (PEVs) with the power grid utilizing the concept of achieving output consensus. The charging/discharging time of all the participating PEVs are separated with respect to time slots and are considered as strategies. In this chapter, we aim to obtain a favorable charging strategy for each grid-connected PEVs in such a way that it satisfies both grid objectives in terms of load profile smoothening and minimizing of load shifting as well as economic and social interests of vehicle owners i.e. a fair share of the rate of charging for all connected PEVs. 
- Chapter 4 develops an AI based power flow prediction methodology for the distribution grid. In this chapter, we used the optimized EV charging and discharging profiles which helped smoothening of load profiles and peak shaving. For this, MLP, RBF network, and CNN were used. For all three neural network, we showed that power flow is predicted with very low error for IEEE 4 bus, IEEE 123 bus, and AEP feeder models. Also, We used some scenarios like topology changes, renewable energy sources integration to predict the power flow for IEEE 123 bus feeder. The results clearly show that we are able to efficiently predict the power flow for such unseen and very new cases using our robust trained CNN model.

\subsection{Proposed Future Work}

- AI for Real-Time Prediction and Smart Grid Automation: To manage and control the new era dynamic grid which is attracting a large number of renewable energy sources, researchers and utilities are looking to implement AI in the power system. Due to the ever increasing penetration of DERs, it is difficult for the utilities to provide a reliable, green, and cost effective energy. This dissertation can be leveraged to further the application of AI to predict the demand, pricing, and automate the smart grid using the weather data, loads, and DERs generation capacities in real time. The AI can also be applied to stabilizing the grid and provide ancillary services.

- AI for Electrification of Transportation: EVs are another key player in the smart grid. EV load forecasting is used to predict the demand. With the random driving pattern of many EV owners and the lack of awareness about the demand response programs may put the grid in danger with the uncoordinated charging. Each of these EVs have their own personal needs and incentives in mind. AI techniques can be developed to solve such EV challenges.

- Feature Extraction: Feature extraction is one of the important concepts of machine learning. According to [123], feature extraction is a type of dimensionality reduction technique which reduces the features from a dataset without loosing the valuable information. Due to the two way communication in a smart grid, a huge amount of data flows and need to be analyzed by grid operators. Feature extraction and AI can be further used to visualize such data coming from Advanced metering infrastructure like smart sensors, phasor measurement units for the smooth operation of the grid. 


\section{LIST OF PUBLICATIONS}

1. D. Tiwari, M. A. Anwar Sheikh, J. Moyalan, M. Sawant, S. K. Solanki, and J. Solanki, "Vehicle-to-Grid Integration for Enhancement of Grid: A Distributed Resource Allocation Approach," in IEEE Access, vol. 8, pp. 175948-175957, 2020, doi: 10.1109/ACCESS.2020.3025170.

2. D. Tiwari, V. Talreja, V. Verma, M. valenti, S. K. Solanki, and J. Solanki, ’Power Flow Analysis using Deep Learning Techniques in a Three Phase Unbalanced Distribution Network", Ready for submission.

3. D. Tiwari, V. Verma, S. K. Solanki and J. Solanki, ”A Primal Dual based Approach to Social Welfare Problem for Electric Vehicle Charging,” 2021, Texas Power and Energy Conference, Accepted.

4. D. Tiwari, S. Chouhan, S. K. Solanki, and J. Solanki, ”Charging coordination of aggregated electrical vehicles for load variance minimization in distribution system," 2017 North American Power Symposium (NAPS), Morgantown, WV, 2017, pp. 1-5, doi: 10.1109/NAPS.2017.8107339.

5. S. Chouhan, D. Tiwari, H. Inan, S. Khushalani-Solanki, and A. Feliachi, ”DER optimization to determine optimum BESS charge/discharge schedule using Linear Programming," 2016 IEEE Power and Energy Society General Meeting (PESGM), Boston, MA, USA, 2016, pp. 1-5, doi: 10.1109/PESGM.2016.7741576.

6. S. Kasani, D. Tiwari, J. Solanki, and S. K. Solanki, ”Coordinated Charging of Plug-in Hybrid Electric Vehicles with Demand Response in a Microgrid”, submitted in Sustainable Cities and Society, Elsevier. 


\section{Bibliography}

[1] Office of Electricity. Grid Modernization and the Smart Grid. " https : / / www . energy . gov/oe/activities/technology-development/grid-modernizationand-smart-grid".

[2] Dan T Ton and Merrill A Smith. "The US department of energy's microgrid initiative”. In: The Electricity Journal 25.8 (2012), pp. 84-94.

[3] K Schneider et al. "Impact assessment of plug-in hybrid vehicles on pacific northwest distribution systems". In: 2008 IEEE Power and Energy Society General Meeting-Conversion and Delivery of Electrical Energy in the 21st Century. IEEE. 2008, pp. 1-6.

[4] Murat Yilmaz, Philip T Krein Murat Yilmaz Philip, and T Krein. "Review of Charging Power Levels and Infrastructure for Plug-In Electric and Hybrid Vehicles and Commentary on Unidirectional Charging”. In: ().

[5] Eric Sortomme et al. "Coordinated charging of plug-in hybrid electric vehicles to minimize distribution system losses". In: IEEE transactions on smart grid 2.1 (2011), pp. 198-205.

[6] Kristien Clement-Nyns, Edwin Haesen, and Johan Driesen. "The impact of charging plugin hybrid electric vehicles on a residential distribution grid". In: IEEE Transactions on power systems 25.1 (2010), pp. 371-380.

[7] International Energy Agency. "https://www. iea.org/reports/global-evout look-2020".

[8] Muhammad Ansari et al. "Coordinated bidding of ancillary services for vehicle-to-grid using fuzzy optimization”. In: IEEE Transactions on Smart Grid 6.1 (2015), pp. 261-270.

[9] Sara Deilami et al. "Real-time coordination of plug-in electric vehicle charging in smart grids to minimize power losses and improve voltage profile". In: IEEE Transactions on Smart Grid 2.3 (2011), pp. 456-467.

[10] Sonia Labatt and Rodney R White. Carbon finance: the financial implications of climate change. Vol. 362. John Wiley and Sons, 2011. 
[11] Michael Angelo A Pedrasa, Ted D Spooner, and Iain F MacGill. "Coordinated scheduling of residential distributed energy resources to optimize smart home energy services". In: IEEE Transactions on Smart Grid 1.2 (2010), pp. 134-143.

[12] Zahra Pooranian, Jemal H Abawajy, Mauro Conti, et al. "Scheduling Distributed Energy Resource Operation and Daily Power Consumption for a Smart Building to Optimize Economic and Environmental Parameters". In: Energies 11.6 (2018), p. 1348.

[13] D. Tiwari et al. "Charging coordination of aggregated electrical vehicles for load variance minimization in distribution system". In: 2017 North American Power Symposium (NAPS). 2017, pp. 1-5. DOI: 10.1109/NAPS.2017.8107339.

[14] Yifeng He, Bala Venkatesh, and Ling Guan. "Optimal scheduling for charging and discharging of electric vehicles". In: IEEE transactions on smart grid 3.3 (2012), pp. 10951105 .

[15] Farivar Fazelpour et al. "Intelligent optimization to integrate a plug-in hybrid electric vehicle smart parking lot with renewable energy resources and enhance grid characteristics". In: Energy Conversion and Management 77 (2014), pp. 250-261.

[16] Mohammad Javad Mirzaei, Ahad Kazemi, and Omid Homaee. "A probabilistic approach to determine optimal capacity and location of electric vehicles parking lots in distribution networks". In: IEEE Transactions on Industrial Informatics 12.5 (2016), pp. 1963-1972.

[17] Pinak J Tulpule et al. "Economic and environmental impacts of a PV powered workplace parking garage charging station”. In: Applied Energy 108 (2013), pp. 323-332.

[18] Shengnan Shao, Manisa Pipattanasomporn, and Saifur Rahman. "Demand response as a load shaping tool in an intelligent grid with electric vehicles". In: IEEE Transactions on Smart Grid 2.4 (2011), pp. 624-631.

[19] Mohammad Reza Norouzi et al. "Mixed integer programming of multi-objective securityconstrained hydro/thermal unit commitment". In: Renewable and Sustainable Energy Reviews 29 (2014), pp. 911-923.

[20] Sridhar Chouhan et al. "Smart mas restoration for distribution system with microgrids". In: 2013 IEEE Power and Energy Society General Meeting. IEEE. 2013, pp. 1-5.

[21] Stanton W Hadley. "Evaluating the impact of plug-in hybrid electric vehicles on regional electricity supplies”. In: 2007 iREP Symposium-Bulk Power System Dynamics and ControlVII. Revitalizing Operational Reliability. IEEE. 2007, pp. 1-12.

[22] Sridhar Chouhan et al. "Hybrid Mas Fault Location, Isolation, and Restoration for Smart Distribution System with Microgrids”. In: 2016 IEEE Power and Energy Society General Meeting. IEEE. 2016, pp. 1-5. 
[23] Sekyung Han, Soohee Han, and Kaoru Sezaki. "Development of an optimal vehicle-togrid aggregator for frequency regulation”. In: IEEE Transactions on smart grid 1.1 (2010), pp. 65-72.

[24] Yuehao Qiu et al. "Intelligent strategy on coordinated charging of PHEV with TOU price". In: 2011 Asia-Pacific Power and Energy Engineering Conference. IEEE. 2011, pp. 1-5.

[25] M Moradijoz et al. "A multi-objective optimization problem for allocating parking lots in a distribution network". In: International Journal of Electrical Power and Energy Systems 46 (2013), pp. 115-122.

[26] Shahab Bahrami and Mostafa Parniani. "Game theoretic based charging strategy for plugin hybrid electric vehicles". In: IEEE Transactions on Smart Grid 5.5 (2014), pp. 23682375.

[27] Gianni Celli et al. "Particle Swarm Optimization for minimizing the burden of electric vehicles in active distribution networks". In: 2012 IEEE Power and Energy Society General Meeting. IEEE. 2012, pp. 1-7.

[28] Sridhar Chouhan et al. "DER optimization to determine optimum BESS charge/discharge schedule using linear programming”. In: 2016 IEEE Power and Energy Society General Meeting (PESGM). IEEE. 2016, pp. 1-5.

[29] M Hadi Amini and Arif Islam. "Allocation of electric vehicles' parking lots in distribution network”. In: ISGT 2014. IEEE. 2014, pp. 1-5.

[30] Hasan Ul Banna et al. "Load variance minimization for coordinated PHEV charging in microgrid”. In: 2016 North American Power Symposium (NAPS). IEEE. 2016, pp. 1-5.

[31] Javad Mohammadi, Gabriela Hug, and Soummya Kar. "A fully distributed cooperative charging approach for plug-in electric vehicles". In: IEEE Transactions on Smart Grid 9.4 (2018), pp. 3507-3518.

[32] Pedram Samadi et al. "Optimal real-time pricing algorithm based on utility maximization for smart grid”. In: 2010 First IEEE International Conference on Smart Grid Communications. IEEE. 2010, pp. 415-420.

[33] Zizhan Zheng and Ness Shroff. "Online welfare maximization for electric vehicle charging with electricity cost". In: Proceedings of the 5th international conference on Future energy systems. ACM. 2014, pp. 253-263.

[34] Yumin Zhang et al. "Unit commitment considering demand response behavior based on utility maximization”. In: 2017 IEEE Conference on Energy Internet and Energy System Integration (EI2). IEEE. 2017, pp. 1-6. 
[35] CC Fung, SCY Ho, and CV Nayar. "Optimisation of a hybrid energy system using simulated annealing technique". In: TENCON'93. Proceedings. Computer, Communication, Control and Power Engineering. 1993 IEEE Region 10 Conference on. Vol. 5. IEEE. 1993, pp. 235-238.

[36] Chin Hwang Lo and Max D Anderson. "Economic dispatch and optimal sizing of battery energy storage systems in utility load-leveling operations". In: IEEE Transactions on Energy Conversion 14.3 (1999), pp. 824-829.

[37] Linni Jian et al. "A scenario of vehicle-to-grid implementation and its double-layer optimal charging strategy for minimizing load variance within regional smart grids". In: Energy conversion and management 78 (2014), pp. 508-517.

[38] Sarika Khushalani, Jignesh M Solanki, and Noel N Schulz. "Optimized Restoration of Unbalancedi? Pub _bookmark Command=" here"? ¿ Distribution Systems". In: IEEE Transactions on Power Systems 22.2 (2007), pp. 624-630.

[39] Ellie Cohen. "https://theclimatecenter.org/opinion-microgrids-could-prevent-need-for-plannedpower-outages/". In: ().

[40] Chao Wei et al. "On optimally reducing power loss in micro-grids with power storage devices". In: IEEE Journal on Selected Areas in Communications 32.7 (2014), pp. 13611370.

[41] M Farrokhifar. "Optimal operation of energy storage devices with RESs to improve efficiency of distribution grids; technical and economical assessment". In: International Journal of Electrical Power and Energy Systems 74 (2016), pp. 153-161.

[42] Talal Alharbi and Kankar Bhattacharya. "Optimal Scheduling of Energy Resources and Management of Loads in Isolated/Islanded Microgrids". In: Canadian Journal of Electrical and Computer Engineering 40.4 (2017), pp. 284-294.

[43] Jin Tan and Yingchen Zhang. "Coordinated control strategy of a battery energy storage system to support a wind power plant providing multi-timescale frequency ancillary services". In: IEEE Transactions on Sustainable Energy 8.3 (2017), pp. 1140-1153.

[44] SY Derakhshandeh et al. "Coordination of generation scheduling with PEVs charging in industrial microgrids”. In: IEEE Trans. Power Syst 28.3 (2013), pp. 3451-3461.

[45] Zhongjing Ma, Ian Hiskens, and Duncan Callaway. "A decentralized MPC strategy for charging large populations of plug-in electric vehicles". In: IFAC Proceedings Volumes 44.1 (2011), pp. 10493-10498.

[46] Krishna Chaitanya Kosaraju et al. "Stability analysis of constrained optimization dynamics via passivity techniques". In: IEEE Control Systems Letters 2.1 (2018), pp. 91-96. 
[47] Kenneth J Arrow, Leonid Hurwicz, and Hirofumi Uzawa. "Studies in linear and non-linear programming”. In: (1958).

[48] Jing Wang and Nicola Elia. "A control perspective for centralized and distributed convex optimization". In: 2011 50th IEEE conference on decision and control and European control conference. IEEE. 2011, pp. 3800-3805.

[49] Kai Ma, Guoqiang Hu, and Costas J Spanos. "Energy management considering load operations and forecast errors with application to HVAC systems". In: IEEE Transactions on Smart Grid 9.2 (2018), pp. 605-614.

[50] OpenEI. OpenEI Database. 2017. URL: http: / / www .en. openei.org/datasets / files/961/pub/ (visited on 2017).

[51] Kevin P Schneider et al. Modern grid initiative distribution taxonomy final report. Tech. rep. Pacific Northwest National Lab.(PNNL), Richland, WA (United States), 2008.

[52] Anderson Hoke et al. "Steady-state analysis of maximum photovoltaic penetration levels on typical distribution feeders". In: IEEE Transactions on Sustainable Energy 4.2 (2013), pp. 350-357.

[53] Roger C Dugan et al. "Roadmap for the IEEE PES test feeders". In: 2009 IEEE/PES Power Systems Conference and Exposition. IEEE. 2009, pp. 1-4.

[54] Techology Roadmap IEA. Electric and Plug-in Hybrid Electric Vehicles. OECD Publishing, 2009.

[55] Daehyun Ban, George Michailidis, and Michael Devetsikiotis. "Demand response control for PHEV charging stations by dynamic price adjustments". In: Innovative Smart Grid Technologies (ISGT), 2012 IEEE PES. IEEE. 2012, pp. 1-8.

[56] Willett Kempton and Jasna Tomić. "Vehicle-to-grid power implementation: From stabilizing the grid to supporting large-scale renewable energy". In: Journal of power sources 144.1 (2005), pp. 280-294.

[57] Derek M Lemoine, Daniel M Kammen, and Alexander E Farrell. "An innovation and policy agenda for commercially competitive plug-in hybrid electric vehicles". In: Environmental Research Letters 3.1 (2008), p. 014003.

[58] Mark Duvall et al. "Environmental assessment of plug-in hybrid electric vehicles". In: EPRI, July 1 (2007).

[59] Michael M Collins and Gerald H Mader. "The timing of EV recharging and its effect on utilities". In: IEEE Transactions on Vehicular Technology 32.1 (1983), pp. 90-97. 
[60] S Rahman and GB Shrestha. "An investigation into the impact of electric vehicle load on the electric utility distribution system". In: IEEE Transactions on Power Delivery 8.2 (1993), pp. 591-597.

[61] Fumiko Koyanagi and Yoshihisa Uriu. "A strategy of load leveling by charging and discharging time control of electric vehicles”. In: IEEE Transactions on Power Systems 13.3 (1998), pp. 1179-1184.

[62] Sitthidet Vachirasricirikul and Issarachai Ngamroo. "Robust controller design of microturbine and electrolyzer for frequency stabilization in a microgrid system with plug-in hybrid electric vehicles". In: International Journal of Electrical Power and Energy Systems 43.1 (2012), pp. 804-811.

[63] Kristien Clement, Edwin Haesen, and Johan Driesen. "Coordinated charging of multiple plug-in hybrid electric vehicles in residential distribution grids". In: Power Systems Conference and Exposition, 2009. PSCE'09. IEEE/PES. IEEE. 2009, pp. 1-7.

[64] Tsai-Hsiang Chen et al. "Evaluation of advantages of an energy storage system using recycled EV batteries". In: International Journal of Electrical Power and Energy Systems 45.1 (2013), pp. 264-270.

[65] Chenye Wu, Hamed Mohsenian-Rad, and Jianwei Huang. "Vehicle-to-aggregator interaction game". In: IEEE Transactions on Smart Grid 3.1 (2012), pp. 434-442.

[66] Saeid Bashash et al. "Plug-in hybrid electric vehicle charge pattern optimization for energy cost and battery longevity". In: Journal of power sources 196.1 (2011), pp. 541-549.

[67] Zhenpo Wang and Shuo Wang. "Grid power peak shaving and valley filling using vehicleto-grid systems". In: IEEE Transactions on power delivery 28.3 (2013), pp. 1822-1829.

[68] Md Jan E Alam, Kashem M Muttaqi, and Danny Sutanto. "A controllable local peakshaving strategy for effective utilization of PEV battery capacity for distribution network support”. In: IEEE Transactions on Industry Applications 51.3 (2014), pp. 2030-2037.

[69] Mithat C Kisacikoglu, Burak Ozpineci, and Leon M Tolbert. "Examination of a PHEV bidirectional charger system for V2G reactive power compensation". In: 2010 Twenty-Fifth Annual IEEE Applied Power Electronics Conference and Exposition (APEC). IEEE. 2010, pp. $458-465$.

[70] Mithat C Kisacikoglu, Metin Kesler, and Leon M Tolbert. "Single-phase on-board bidirectional PEV charger for V2G reactive power operation". In: IEEE Transactions on Smart Grid 6.2 (2014), pp. 767-775.

[71] Kristien Clement-Nyns, Edwin Haesen, and Johan Driesen. "The impact of vehicle-to-grid on the distribution grid”. In: Electric Power Systems Research 81.1 (2011), pp. 185-192. 
[72] Sekyung Han and Soohee Han. "Development of short-term reliability criterion for frequency regulation under high penetration of wind power with vehicle-to-grid support". In: Electric Power Systems Research 107 (2014), pp. 258-267.

[73] Ricardo Jorge Bessa and MA Matos. "Optimization models for an EV aggregator selling secondary reserve in the electricity market”. In: Electric Power Systems Research 106 (2014), pp. 36-50.

[74] Hui Liu et al. "Vehicle-to-grid control for supplementary frequency regulation considering charging demands". In: IEEE Transactions on Power Systems 30.6 (2014), pp. 3110-3119.

[75] NZ Xu and CY Chung. "Reliability evaluation of distribution systems including vehicle-tohome and vehicle-to-grid”. In: IEEE transactions on power systems 31.1 (2015), pp. 759768.

[76] Hao Xing et al. "Decentralized optimal scheduling for charging and discharging of plug-in electric vehicles in smart grids". In: IEEE Transactions on Power Systems 31.5 (2015), pp. $4118-4127$.

[77] Mithat C Kisacikoglu, Fatih Erden, and Nuh Erdogan. "Distributed control of PEV charging based on energy demand forecast". In: IEEE Transactions on Industrial Informatics 14.1 (2017), pp. 332-341.

[78] Farshad Rassaei, Wee-Seng Soh, and Kee-Chaing Chua. "Demand response for residential electric vehicles with random usage patterns in smart grids". In: IEEE Transactions on Sustainable Energy 6.4 (2015), pp. 1367-1376.

[79] Azar Rahimi et al. "A simple and effective approach for peak load shaving using Battery Storage Systems”. In: 2013 North American Power Symposium (NAPS). IEEE. 2013, pp. 15 .

[80] I Komang Adi Aswantara, Kab Seok Ko, and Dan Keun Sung. "A dynamic point of preferred operation (PPO) scheme for charging electric vehicles in a residential area". In: 2013 International Conference on Connected Vehicles and Expo (ICCVE). IEEE. 2013, pp. 201206.

[81] Nuh Erdogan, Fatih Erden, and Mithat Kisacikoglu. "A fast and efficient coordinated vehicle-to-grid discharging control scheme for peak shaving in power distribution system". In: Journal of Modern Power Systems and Clean Energy 6.3 (2018), pp. 555-566.

[82] Sonja Stüdli et al. "Optimal real-time distributed V2G and G2V management of electric vehicles”. In: International Journal of Control 87.6 (2014), pp. 1153-1162.

[83] Pooya Rezaei, Jeff Frolik, and Paul DH Hines. "Packetized plug-in electric vehicle charge management". In: IEEE Transactions on Smart Grid 5.2 (2014), pp. 642-650. 
[84] J Moyalan et al. "Electric Vehicle-Power Grid Incorporation Using Distributed Resource Allocation Approach". In: 2019 18th European Control Conference (ECC). IEEE. 2019, pp. 3034-3039.

[85] M Sawant et al. "Power Grid Load Management using Electric Vehicles: A Distributed Resource Allocation Approach”. In: 2019 27th Mediterranean Conference on Control and Automation (MED). IEEE. 2019, pp. 428-433.

[86] Andres Ovalle et al. "Escort evolutionary game dynamics approach for integral load management of electric vehicle fleets". In: IEEE Transactions on Industrial Electronics 64.2 (2017), pp. 1358-1369.

[87] Hassan K Khalil and Jessy W Grizzle. Nonlinear systems. Vol. 3. Prentice hall Upper Saddle River, NJ, 2002.

[88] Germán Darío Obando Bravo. "Distributed methods for resource allocation: a passivity based approach". PhD thesis. Ecole des Mines de Nantes, 2015.

[89] Jennifer Cardona, Thomas Gill, and Siobhan Powell. "Machine Learning Models for Inverse Power Flow in the Grid". In: (2017).

[90] Nishesh Kumar et al. "Application of artificial neural networks to load flow solutions". In: TENCON'91. Region 10 International Conference on EC3-Energy, Computer, Communication and Control Systems. Vol. 1. IEEE. 1991, pp. 199-203.

[91] Mohsen Hayati and Yazdan Shirvany. "Artificial neural network approach for short term load forecasting for Illam region”. In: World Academy of Science, Engineering and Technology 28 (2007), pp. 280-284.

[92] Nahi Kandil et al. "An efficient approach for shorterm load forecasting using artificial neural networks". In: 2006 IEEE International Symposium on Industrial Electronics. Vol. 3. IEEE. 2006, pp. 1928-1932.

[93] Dong C Park et al. "Electric load forecasting using an artificial neural network". In: IEEE transactions on Power Systems 6.2 (1991), pp. 442-449.

[94] Ping-Feng Pai and Wei-Chiang Hong. "Support vector machines with simulated annealing algorithms in electricity load forecasting". In: Energy Conversion and Management 46.17 (2005), pp. 2669-2688.

[95] Seunghyoung Ryu, Jaekoo Noh, and Hongseok Kim. "Deep neural network based demand side short term load forecasting”. In: Energies 10.1 (2016), p. 3. 
[96] Ali Dehghan Banadaki, Taufik Taufik, and Ali Feliachi. "Big Data Analytics in a DayAhead Electricity Price Forecasting Using TensorFlow in Restructured Power Systems". In: 2018 International Conference on Computational Science and Computational Intelligence (CSCI). 2018.

[97] Elena Mocanu, Phuong H Nguyen, and Madeleine Gibescu. "Deep learning for power system data analysis”. In: Big data application in power systems. Elsevier, 2018, pp. 125158.

[98] Yize Chen, Yushi Tan, and Deepjyoti Deka. "Is Machine Learning in Power Systems Vulnerable?" In: 2018 IEEE International Conference on Communications, Control, and Computing Technologies for Smart Grids (SmartGridComm). IEEE. 2018, pp. 1-6.

[99] Yixing Wang, Meiqin Liu, and Zhejing Bao. "Deep learning neural network for power system fault diagnosis". In: 2016 35th Chinese Control Conference (CCC). IEEE. 2016, pp. 6678-6683.

[100] Wei Zheng, Desheng Hu, and Jing Wang. "Fault localization analysis based on deep neural network". In: Mathematical Problems in Engineering 2016 (2016).

[101] Wenting Li et al. "Real-time Fault Localization in Power Grids With Convolutional Neural Networks". In: arXiv preprint arXiv:1810.05247 (2018).

[102] Kursat Rasim Mestav, Jaime Luengo-Rozas, and Lang Tong. "Bayesian State Estimation for Unobservable Distribution Systems via Deep Learning”. In: arXiv preprint arXiv:1811.02756 (2018).

[103] DHMW Thukaram, HM Wijekoon Banda, and Jovitha Jerome. "A robust three phase power flow algorithm for radial distribution systems". In: Electric Power Systems Research 50.3 (1999), pp. 227-236.

[104] William H Kersting. Distribution system modeling and analysis. CRC press, 2017.

[105] Ray D Zimmerman and Hsiao-Dong Chiang. "Fast decoupled power flow for unbalanced radial distribution systems". In: IEEE Transactions on Power systems 10.4 (1995), pp. 20452052.

[106] Jen-Hao Teng. "A direct approach for distribution system load flow solutions". In: IEEE Transactions on power delivery 18.3 (2003), pp. 882-887.

[107] Roger C Dugan. "Reference guide". In: The Open Distribution System Simulator (OpenDSS). EPRI (2016). 
[108] S. Khushalani, J. M. Solanki, and N. N. Schulz. "Optimized Restoration of Unbalanced Distribution Systems”. In: IEEE Transactions on Power Systems 22.2 (2007), pp. 624630. DOI: $10.1109 /$ TPWRS.2007.894866.

[109] Hossein Akhavan-Hejazi and Hamed Mohsenian-Rad. "Power systems big data analytics: An assessment of paradigm shift barriers and prospects". In: Energy Reports 4 (2018), pp. 91-100.

[110] Olga Bolgurtseva Yulia Gavrilova. What Is Data Preprocessing in ML? "https : / / https://serokell.io/blog/data-preprocessing\#data-cleaning".

[111] Davis Montenegro, Miguel Hernandez, and Gustavo A Ramos. "Real time OpenDSS framework for distribution systems simulation and analysis". In: 2012 Sixth IEEE/PES Transmission and Distribution: Latin America Conference and Exposition (TandD-LA). IEEE. 2012, pp. 1-5.

[112] Y. Lecun et al. "Gradient-based learning applied to document recognition". In: Proceedings of the IEEE 86.11 (1998), pp. 2278-2324. ISSN: 0018-9219. DOI: $10.1109 / 5$. 726791.

[113] MissingLink.ai. 7 Types of Neural Network Activation Functions: How to Choose? URL: https : / / missinglink . ai / guides / neural-network-concepts / 7 types-neural-network-activation-functions-right/.

[114] Prajit Ramachandran, Barret Zoph, and Quoc V. Le. "Searching for Activation Functions". In: CoRR abs/1710.05941 (2017). URL: http: / / arxiv . org/abs/1710 . 05941.

[115] Richard O Duda, Peter E Hart, and David G Stork. Pattern classification. John Wiley and Sons, 2012.

[116] Diederik P. Kingma and Jimmy Ba. “Adam: A Method for Stochastic Optimization”. In: CoRR abs/1412.6980 (2015).

[117] Introduction to TensorFlow. "https: / / www.tensorflow.org/".

[118] IEEE PES Distribution system analysis subcommittee. "http : / / www . ewh. ieee. org/soc/pes/dsacom/testfeeders/index.html". 2011.

[119] New York Independent State Operator (ISO). Energy Market Operational Data. "https : //www.nyiso.com/load-data".

[120] Envisioning a Renewable Electricity Future for the United States. "https : / / www . energy ·gov/ eere / analysis / downloads / envisioning-renewableelectricity-future-united-states".

[121] Manajit Sengupta et al. "The national solar radiation data base (NSRDB)". In: Renewable and Sustainable Energy Reviews 89 (2018), pp. 51-60. 
[122] D. Tiwari et al. "Vehicle-to-Grid Integration for Enhancement of Grid: A Distributed Resource Allocation Approach”. In: IEEE Access 8 (2020), pp. 175948-175957. DOI: 10 . $1109 /$ ACCESS.2020.3025170.

[123] Anke Meyer-Baese and Volker Schmid. "Chapter 2 - Feature Selection and Extraction". In: Pattern Recognition and Signal Analysis in Medical Imaging (Second Edition). Ed. by Anke Meyer-Baese and Volker Schmid. Second Edition. Oxford: Academic Press, 2014, pp. 21-69. ISBN: 978-0-12-409545-8. DOI: https : / / doi . org/10 . 1016/B9780-12-409545-8.00002-9. URL: https: / / www. sciencedirect. com/ science/article/pii/B9780124095458000029. 


\section{Appendices}




\section{Appendix A}

\section{Abbreviation}

$E_{t}^{B E S S}=$ Energy stored in BESS at time $t$

$P_{j t, c h}^{B E S S}=$ BESS charging power

$P_{j t, d i s}^{B E S S}=\mathrm{BESS}$ discharging power

$S o C_{\text {jmin }}^{B E S S}=$ Lower limit of BESS SoC

$S o C_{\text {jmax }}^{B E S S}=$ Upper limit of BESS SoC

$B=$ Number of BESS in the microgrid

$D=$ Number of DERs in the microgrid

$E_{\min }^{B E S S}=$ Minimum energy allowed to be stored in BESS

$E_{\max }^{B E S S}=$ Maximum energy allowed to be stored in BESS

$E_{r, t}^{E V}=$ Energy stored in $r^{t h} \mathrm{EV}$ at time $\mathrm{t}$

$P_{r, t}^{E V}=$ Charging power of $r^{t h} \mathrm{EV}$ at time $\mathrm{t}$

$\eta_{c h}^{E V}=$ Charging efficiency of EV

$P_{i t}=$ Output power from $i^{t h}$ DER

$P_{i}^{\min }=$ Lower limit of output power from $i^{\text {th }}$ DER

$P_{i}^{\max }=\mathrm{Upper}$ limit of output power from $i^{\text {th }} \mathrm{DER}$

$c_{i}=$ Operating cost for $i^{\text {th }}$ DER

$c_{j}=$ Operating cost for $j^{t h} \mathrm{BESS}$

$c_{r}=$ Charging cost for $r^{t h}$ BESS

$C_{t}=$ Electricity price at $t^{t h}$ hour

$P_{G t}=$ Power from grid

$P_{d t}=$ System power demand

$N=$ Number of PHEVs

$S_{s}=$ time instant at which charging of n-th PHEV starts

$S_{e}=$ time instant at which chraging of n-th PHEV ends

$H_{t}=$ household load power at the t-th time instant

$C h_{t, n}=$ charging profile of $\mathrm{n}$-th PHEV at the $\mathrm{t}$-th time

$H_{t, \text { max }}=$ maximum power of the grid at $\mathrm{t}$-th time 
$C h_{n, \max }=$ maximum power of $\mathrm{n}$-th charger at the $\mathrm{t}$-th time slot

$M_{t}^{h}=$ household average power for whole day

$S O C_{n, \min }=$ lower bound of state of charge of the nth PHEV battery

$S O C_{n, \max }=$ upper bound of charge of the n-th PHEV battery

$B c_{s}=$ initial charge available in PHEV when connected to charger

$B c_{e}=$ charge available in PHEV when disconnected from charger

$K_{n}=$ PHEV battery capacity

$y_{i}(k)=$ State of charge $(\mathrm{SoC})$ of the $i^{t h}$ vehicle at $k^{t h}$ time step

$\alpha_{i}=$ Charger efficiency of $i^{t h}$ vehicle

$B_{i}=$ Battery size of $i^{\text {th }}$ vehicle

$x_{t}=$ Charging strategy

$q=$ Maximum allocated power

$\gamma=$ Trade-off parameter

$b_{i}=$ Constant parameter

$\lambda=$ Charging price

$\rho_{1}, \rho_{2}, \rho_{3}=$ Known coefficients of cost function

$\mathcal{S}$ Set of nodes of a multi-agent system

$\mathcal{L}$ Set of edges connecting the nodes of a multi-agent system

$\mathcal{A}$ A nonnegative matrix whose elements satisfy the following: $a_{k j}=1$ if $(k, j) \in \mathcal{L} ; a_{k j}=0$ if $(k, j) \notin \mathcal{L}$

$\mathcal{N}_{k}$ Set of neighbours of node $k$

$A_{k}$ Total active power supply of grid at $k^{\text {th }}$ time slot

$R_{k}$ Total reactive power supply of grid at $k^{\text {th }}$ time slot

$\beta$ Barrier function

$x_{k}^{i}$ Active power charging strategy at $k^{\text {th }}$ time step of $i^{\text {th }}$ PEV

$y_{k}^{i}$ Reactive power charging strategy at $k^{\text {th }}$ time step of $i^{\text {th }} \mathrm{PEV}$

$K^{i}$ Number of time steps allotted to $i^{\text {th }}$ PEV

$s o c_{K}^{i}$ Desired state of charge of $i^{\text {th }} \mathrm{PEV}$

$s o c_{o}^{i}$ Initial state of charge of $i^{\text {th }} \mathrm{PEV}$

$\overline{s o c}_{o}^{i}$ Upper limit of $i^{\text {th }}$ PEV charger

$\underline{S O C}_{o}^{i}$ Lower limit of $i^{t h}$ PEV charger

$t_{k}^{i}$ Time width of $k^{\text {th }}$ time step of $i^{\text {th }}$ PEV

$\bar{p}^{i}$ Nominal power of $i^{\text {th }}$ PEV charger

$\mu$ Commitment factor controlled by the PEV owner

$\eta$ Smoothing factor controlled by the power grid manager

$s^{i}$ Auxillary slack variable of $i^{t h}$ PEV 
$Q^{i}$ Total reactive power available of $i^{\text {th }} \mathrm{PEV}$

$\bar{q}_{k}^{i}$ Available reactive power of of $i^{t h} \mathrm{PEV}$ at $k^{\text {th }}$ time step 